\title{
Self-Assembled Redox-Active Tetraruthenium Macrocycles with Large Intracyclic Cavities
}

Daniel Fink,$^{\dagger}$ Nicole Orth, ${ }^{\dagger}$ Viktoria Ebel,${ }^{\dagger}$ Franciska S. Gogesch, ${ }^{\dagger}$ Anne Staiger, ${ }^{\dagger}$ Michael Linseis, ${ }^{\dagger}$ Ivana Ivanović-Burmazović ${ }^{\ddagger}$ and Rainer F. Winter*, ${ }^{*}$

${ }^{\dagger}$ Fachbereich Chemie, Universität Konstanz, Universitätsstraße 10, 78457 Konstanz, Germany

* Department Chemie und Pharmazie, Friedrich-Alexander-Universität Erlangen-Nürnberg, Egerlandstraße 1,

91058 Erlangen, Germany

\section{Supporting Information}

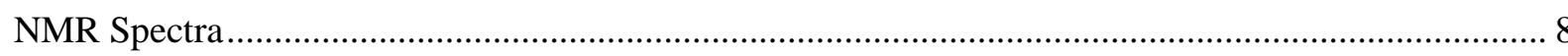

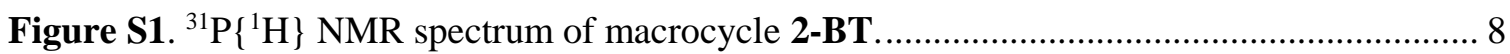

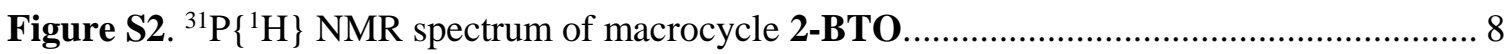

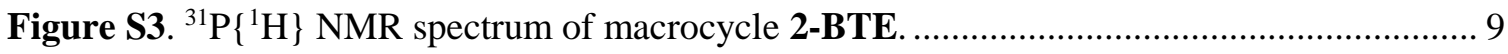

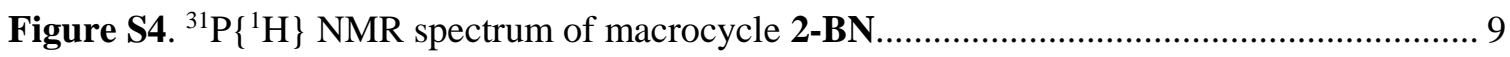

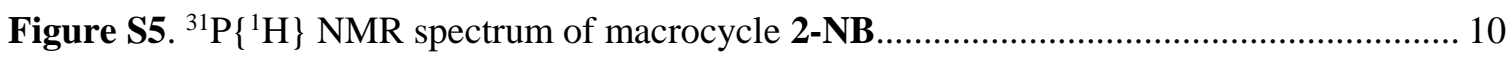

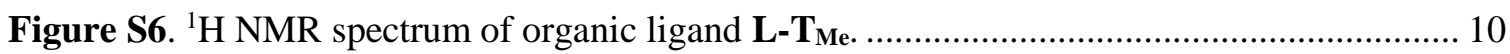

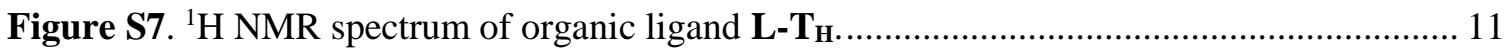

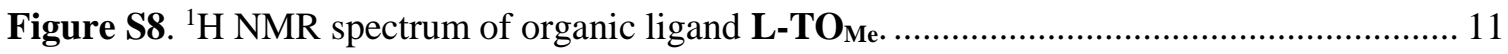

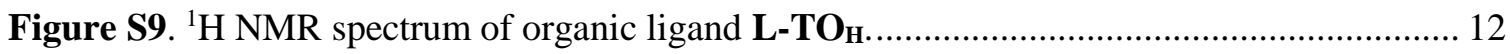

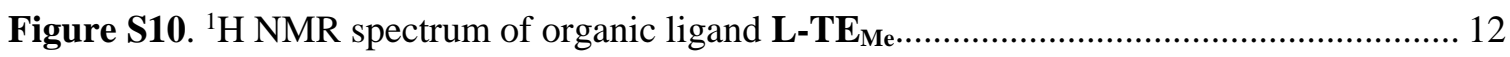

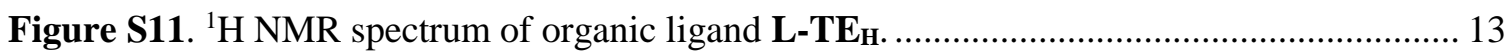

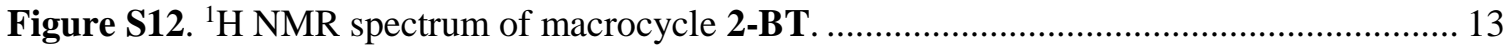

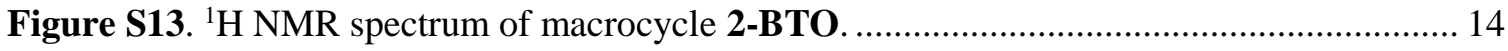

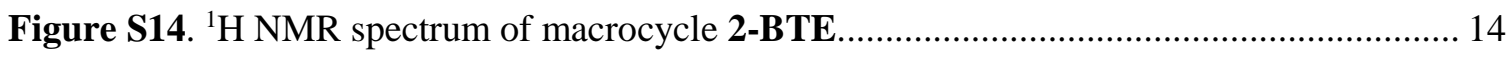

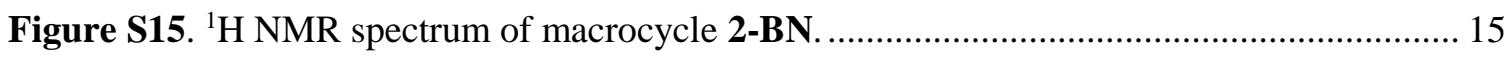

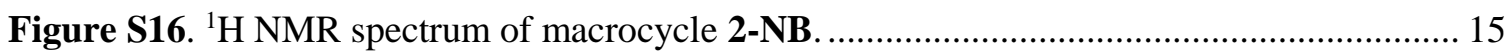

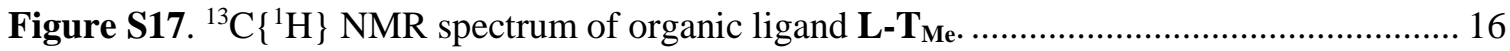

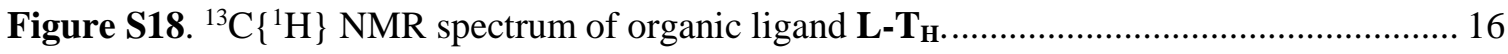

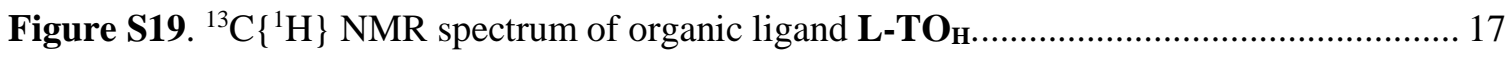

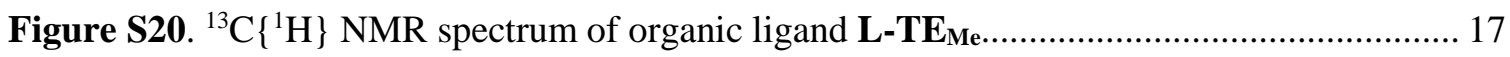




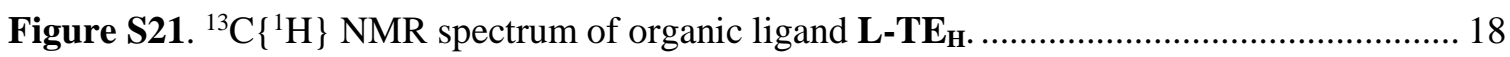

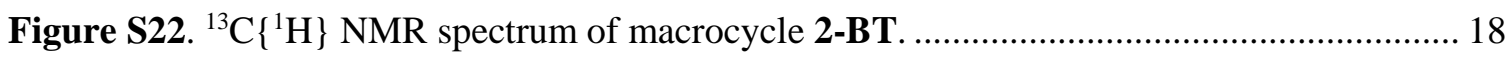

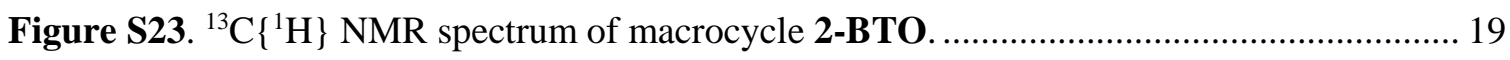

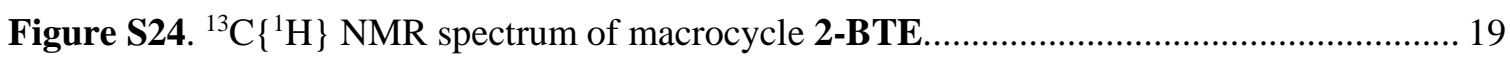

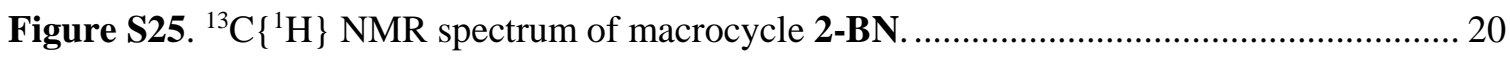

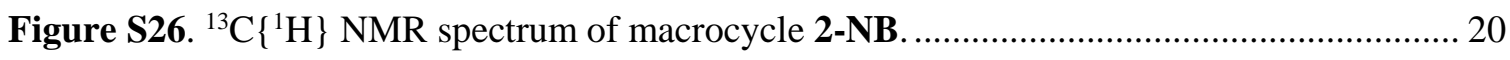

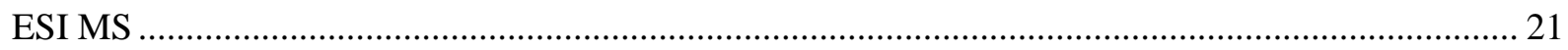

Figure S27. ESI Mass spectrum of macrocycle 2-BT (top); magnification of $[\mathrm{M}]^{2+}$ peak with calculated isotopic pattern (bottom).

Figure S28. ESI Mass spectrum of macrocycle 2-BTO (top), magnification of $[\mathrm{M}]^{2+}$ peak with calculated isotopic pattern (bottom).

Figure S29. ESI Mass spectrum of macrocycle 2-BTE (top), magnification of $[\mathrm{M}]^{2+}$ peak with calculated isotopic pattern (bottom).

Figure S30. ESI Mass spectrum of macrocycle 2-BN (top), magnification of $[\mathrm{M}]^{2+}$ peak with calculated isotopic pattern (bottom).

Figure S31. ESI Mass spectrum of macrocycle 2-NB (top), magnification of $[\mathrm{M}]^{2+}$ peak with

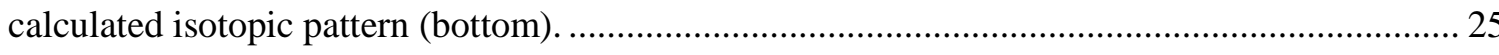

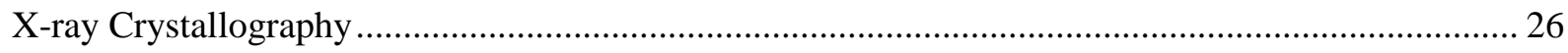

Compound 2-BT:

Figure S32. Structure of macrocycle 2-BT as the benzene hexadecasolvate with atom numbering. Benzene solvate molecules as well as hydrogen atoms have been removed for clarity reasons.

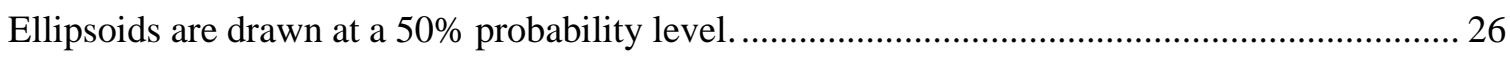

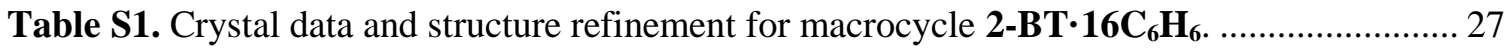

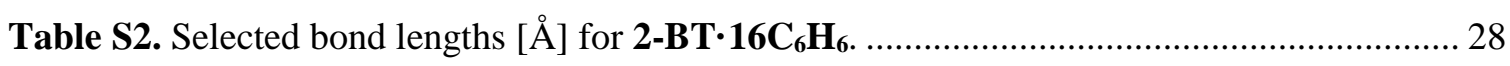

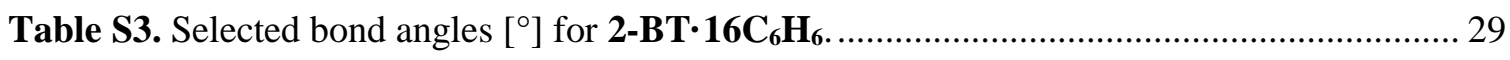

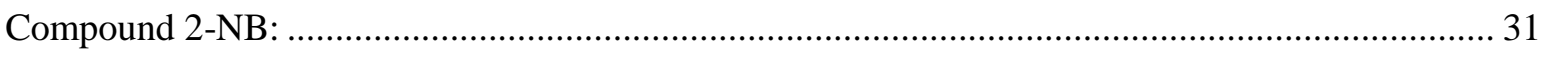

Figure S33. Structure of macrocycle 2-NB as the benzene hexasolvate with atom numbering.

Benzene solvate molecules as well as hydrogen atoms have been removed for clarity reasons.

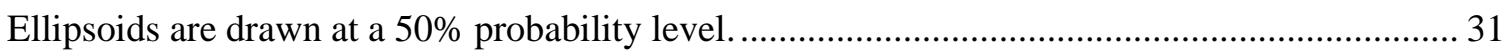

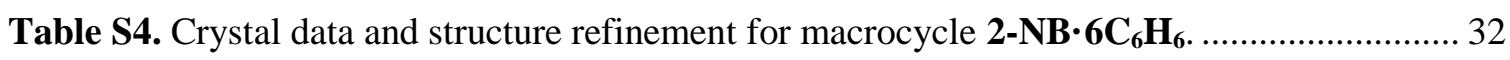

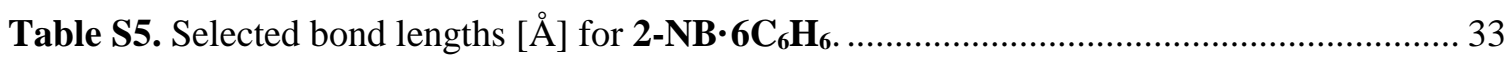


Figure S34. Packing diagram of individual 2-NB molecules in the crystal. Benzene solvate molecules as well as hydrogen atoms have been removed for clarity reasons. Ellipsoids are drawn at a $50 \%$ probability level.

Figure S35. Hydrogen bonding motifs in the molecular structure of metallacycle 2-NB. Benzene solvate molecules have been removed for clarity reasons. Ellipsoids are drawn at a 50\% probability level 36

Figure S36. Packing diagram of individual 2-BT molecules in the crystal. Benzene solvate molecules as well as hydrogen atoms have been removed for clarity reasons. Ellipsoids are drawn at a $50 \%$ probability level.

Figure S37. Hydrogen bonding motif in the molecular structure of metallacycle 2-BT. Benzene solvate molecules have been removed for clarity reasons. Ellipsoids are drawn at a 50\% probability level

Figure S38. DFT-optimized structures of model compounds 2-NB ${ }^{\mathrm{Me}}$ (left) and 2-BT ${ }^{\mathrm{Me}}$ (right). 38

Cyclic Voltammetry

Compound 2-BT

Figure S39. Cyclic voltammograms for the first composite wave of macrocycle 2-BT at $v=100 \mathrm{mV} / \mathrm{s}$ (left) and at $v=25,50,100,200,400,600,800,1000 \mathrm{mV} / \mathrm{s}$ (right) in $\mathrm{CH}_{2} \mathrm{Cl}_{2} /{ }^{n} \mathrm{Bu}_{4} \mathrm{NBAr}^{\mathrm{F} 24}$.

Figure S40. Square wave voltammogram of macrocycle 2-BT (left) with deconvolution (right), measured in $\mathrm{CH}_{2} \mathrm{Cl}_{2} /{ }^{n} \mathrm{Bu}_{4} \mathrm{NBAr}^{\mathrm{F} 24}$.

Compound 2-BTO:

Figure S41. Cyclic voltammograms for the first composite wave of macrocycle 2-BTO at $v=100 \mathrm{mV} / \mathrm{s}$ (left) and at $v=25,50,100,200,400,600,800,1000 \mathrm{mV} / \mathrm{s}$ (right) in $\mathrm{CH}_{2} \mathrm{Cl}_{2} /{ }^{n} \mathrm{Bu}_{4} \mathrm{NBAr}^{\mathrm{F} 24}$. 40

Figure S42. Cyclic voltammograms for the first two composite waves of macrocycle 2-BTO at $v=100 \mathrm{mV} / \mathrm{s}$ (left) and at $v=25,50,100,200,400,600,800,1000 \mathrm{mV} / \mathrm{s}$ (right) in $\mathrm{CH}_{2} \mathrm{Cl}_{2} /{ }^{n} \mathrm{Bu}_{4} \mathrm{NBAr}^{\mathrm{F} 24}$.

Table S7. Data of the cyclovoltammetric measurements for macrocycle 2-BTO at different scan rates, measured in $\mathrm{CH}_{2} \mathrm{Cl}_{2} /{ }^{n} \mathrm{Bu}_{4} \mathrm{NBAr}^{\mathrm{F} 24}$.

Figure S43. Square wave voltammogram of macrocycle 2-BTO (left) with deconvolution (right), measured in $\mathrm{CH}_{2} \mathrm{Cl}_{2} /{ }^{n} \mathrm{Bu}_{4} \mathrm{NBAr}^{\mathrm{F} 24}$ 
Figure S44. Cyclic voltammograms for the first composite wave of macrocycle 2-BTE at $v=100 \mathrm{mV} / \mathrm{s}$ (left) and at $v=25,50,100,200,400,600,800,1000 \mathrm{mV} / \mathrm{s}$ (right) in $\mathrm{CH}_{2} \mathrm{Cl}_{2} /{ }^{n} \mathrm{Bu}_{4} \mathrm{NBAr}^{\mathrm{F} 24}$

Figure S45. Cyclic voltammograms for the first two composite waves of macrocycle 2-BTE at $v=100 \mathrm{mV} / \mathrm{s}$ (left) and at $v=25,50,100,200,400,600,800,1000 \mathrm{mV} / \mathrm{s}$ (right) in $\mathrm{CH}_{2} \mathrm{Cl}_{2} /{ }^{n} \mathrm{Bu}_{4} \mathrm{NBAr}^{\mathrm{F} 24}$.

Table S8. Data of the cyclovoltammetric measurements for macrocycle 2-BTE at different scan rates, measured in $\mathrm{CH}_{2} \mathrm{Cl}_{2} /{ }^{n} \mathrm{Bu}_{4} \mathrm{NBAr}^{\mathrm{F} 24}$.

Figure S46. Square wave voltammogram of macrocycle 2-BTE (left) with deconvolution (right), measured in $\mathrm{CH}_{2} \mathrm{Cl}_{2} /{ }^{n} \mathrm{Bu}_{4} \mathrm{NBAr}^{\mathrm{F} 24}$.

Figure S47. Cyclic voltammograms for the first composite wave of macrocycle 2-BN at $v=100 \mathrm{mV} / \mathrm{s}$ (left) and at $v=25,50,100,200,400,600,800,1000 \mathrm{mV} / \mathrm{s}$ (right) in $\mathrm{CH}_{2} \mathrm{Cl}_{2} /{ }^{n} \mathrm{Bu}_{4} \mathrm{NBAr}^{\mathrm{F} 24}$.

Figure S48. Cyclic voltammograms for the first two composite waves of macrocycle 2-BN at $v=100 \mathrm{mV} / \mathrm{s}$ (left) and at $v=25,50,100,200,400,600,800,1000 \mathrm{mV} / \mathrm{s}$ (right) in $\mathrm{CH}_{2} \mathrm{Cl}_{2} /{ }^{n} \mathrm{Bu}_{4} \mathrm{NBAr}^{\mathrm{F} 24}$.

Figure S49. Cyclic voltammograms for the first three composite waves of macrocycle 2-BN at $v=100 \mathrm{mV} / \mathrm{s}$ (left) and at $v=25,50,100,200,400,600,800,1000 \mathrm{mV} / \mathrm{s}$ (right) in $\mathrm{CH}_{2} \mathrm{Cl}_{2} /{ }^{n} \mathrm{Bu}_{4} \mathrm{NBAr}^{\mathrm{F} 24}$.

Table S9. Data of the cyclovoltammetric measurements for macrocycle 2-BN at different scan rates, measured in $\mathrm{CH}_{2} \mathrm{Cl}_{2} /{ }^{n} \mathrm{Bu}_{4} \mathrm{NBAr}^{\mathrm{F} 24}$.

Figure S50. Square wave voltammogram of macrocycle 2-BN (left) with deconvolution (right), measured in $\mathrm{CH}_{2} \mathrm{Cl}_{2} /{ }^{n} \mathrm{Bu}_{4} \mathrm{NBAr}^{\mathrm{F} 24}$

Figure S51. Cyclic voltammograms for the first composite wave of macrocycle 2-NB at $v=100 \mathrm{mV} / \mathrm{s}$ (left) and at $v=25,50,100,200,400,600,800,1000 \mathrm{mV} / \mathrm{s}$ (right) in $\mathrm{CH}_{2} \mathrm{Cl}_{2} /{ }^{n} \mathrm{Bu}_{4} \mathrm{NBAr}^{\mathrm{F} 24}$ 46

Figure S52. Cyclic voltammograms for the first two composite waves of macrocycle 2-NB at $v=100 \mathrm{mV} / \mathrm{s}$ (left) and at $v=25,50,100,200,400,600,800,1000 \mathrm{mV} / \mathrm{s}$ (right) in $\mathrm{CH}_{2} \mathrm{Cl}_{2} /{ }^{n} \mathrm{Bu}_{4} \mathrm{NBAr}^{\mathrm{F} 24}$. 
Figure S53. Cyclic voltammograms for the first two composite waves of macrocycle 2-NB at $v=100 \mathrm{mV} / \mathrm{s}$ (left) and at $v=25,50,100,200,400,600,800,1000 \mathrm{mV} / \mathrm{s}$ (right) in $\mathrm{CH}_{2} \mathrm{Cl}_{2} /{ }^{n} \mathrm{Bu}_{4} \mathrm{NBAr}^{\mathrm{F} 24}$.

Table S10. Data of the cyclovoltammetric measurements for macrocycle 2-NB at different scan rates, measured in $\mathrm{CH}_{2} \mathrm{Cl}_{2} /{ }^{n} \mathrm{Bu}_{4} \mathrm{NBAr}^{\mathrm{F} 24}$.

Figure S54. Square wave voltammogram of macrocycle 2-NB (left) with deconvolution (right), measured in $\mathrm{CH}_{2} \mathrm{Cl}_{2} /{ }^{n} \mathrm{Bu}_{4} \mathrm{NBAr}^{\mathrm{F} 24}$.

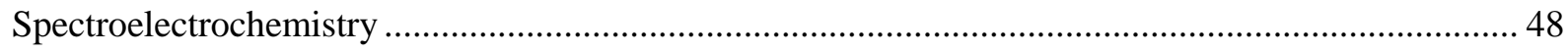

Compound 2-BT:

Figure S55. Changes in the IR spectra of $\mathbf{2 - B T}$ upon oxidation to $\mathbf{2}-\mathbf{B T}^{2+}$, measured in $\mathrm{CH}_{2} \mathrm{Cl}_{2} /{ }^{n} \mathrm{Bu}_{4} \mathrm{NPF}_{6}$. 48

Figure S56. Changes in the IR spectra of $\mathbf{2}-\mathbf{B T}^{2+}$ upon oxidation to $\mathbf{2 - B \mathbf { B } ^ { 4 + }}$, measured in $\mathrm{CH}_{2} \mathrm{Cl}_{2} /{ }^{n} \mathrm{Bu}_{4} \mathrm{NPF}_{6}$. 48

Figure S57. Deconvolutions of the Carbonyl bands of 2-BT ${ }^{2+}$ (left) and $\mathbf{2 - B T ^ { 4 + }}$ (right). 48

Figure S58. Changes in the UV/Vis/NIR spectra of 2-BT upon oxidation to 2-B $\mathbf{T}^{2+}$ (left) and deconvolution (right), measured in $\mathrm{CH}_{2} \mathrm{Cl}_{2} /{ }^{n} \mathrm{Bu}_{4} \mathrm{NPF}_{6}$.

Figure S59. Changes in the UV/Vis/NIR spectra of $\mathbf{2}-\mathbf{B T}^{\mathbf{2 +}}$ upon oxidation to $\mathbf{2}-\mathbf{B T}^{\mathbf{4 +}}$ (left) and deconvolution (right), measured in $\mathrm{CH}_{2} \mathrm{Cl}_{2} /{ }^{n} \mathrm{Bu}_{4} \mathrm{NPF}_{6}$

Compound 2-BTO:

Figure S60. Changes in the IR spectra of 2-BTO upon oxidation to $\mathbf{2}-\mathbf{B T O}^{2+}$, measured in $\mathrm{CH}_{2} \mathrm{Cl}_{2} /{ }^{n} \mathrm{Bu}_{4} \mathrm{NPF}_{6}$

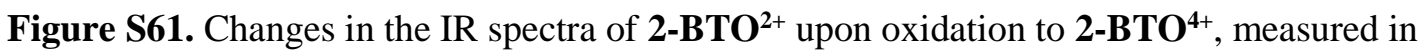
$\mathrm{CH}_{2} \mathrm{Cl}_{2} /{ }^{n} \mathrm{Bu}_{4} \mathrm{NPF}_{6}$. 50

Figure S62. Deconvolutions of the Carbonyl bands of 2-BTO ${ }^{2+}$ (left) and 2-BTO ${ }^{4+}$ (right)....... 50

Figure S63. Changes in the UV/Vis/NIR spectra of 2-BTO upon oxidation to 2-BTO ${ }^{2+}$ (left) and deconvolution (right), measured in $\mathrm{CH}_{2} \mathrm{Cl}_{2} /{ }^{n} \mathrm{Bu}_{4} \mathrm{NPF}_{6}$.

Figure S64. Changes in the UV/Vis/NIR spectra of 2-BTO ${ }^{2+}$ upon oxidation to 2-BTO ${ }^{4+}$ (left) and deconvolution (right), measured in $\mathrm{CH}_{2} \mathrm{Cl}_{2} /{ }^{n} \mathrm{Bu}_{4} \mathrm{NPF}_{6}$.

Compound 2-BTE:

Figure S65. Changes in the IR spectra of 2-BTE upon oxidation to 2-BTE ${ }^{2+}$, measured in $\mathrm{CH}_{2} \mathrm{Cl}_{2} /{ }^{n} \mathrm{Bu}_{4} \mathrm{NPF}_{6}$ 
Figure S66. Changes in the IR spectra of $\mathbf{2}-\mathbf{B T E}^{2+}$ upon oxidation to $\mathbf{2 - B T E}{ }^{4+}$, measured in $\mathrm{CH}_{2} \mathrm{Cl}_{2} /{ }^{n} \mathrm{Bu}_{4} \mathrm{NPF}_{6}$

Figure S67. Deconvolutions of the Carbonyl bands of 2-BTE ${ }^{2+}$ (left) and 2-BTE ${ }^{4+}$ (right)........ 52

Figure S68. Changes in the UV/Vis/NIR spectra of 2-BTE ${ }^{2+}$ upon oxidation to 2-BTE ${ }^{4+}$ (left) and deconvolution (right), measured in $\mathrm{CH}_{2} \mathrm{Cl}_{2} /{ }^{n} \mathrm{Bu}_{4} \mathrm{NPF}_{6}$.

Figure S69. Changes in the UV/Vis/NIR spectra of 2-BTE ${ }^{2+}$ upon oxidation to $\mathbf{2}-\mathbf{B T E}^{4+}$ (left) and deconvolution (right), measured in $\mathrm{CH}_{2} \mathrm{Cl}_{2} /{ }^{n} \mathrm{Bu}_{4} \mathrm{NPF}_{6}$.

Compound 2-BN:

Figure S70. Changes in the IR spectra of $\mathbf{2 - B N}$ upon oxidation to $\mathbf{2}-\mathbf{B N}^{2+}$, measured in $\mathrm{CH}_{2} \mathrm{Cl}_{2} /{ }^{n} \mathrm{Bu}_{4} \mathrm{NPF}_{6}$

Figure S71. Changes in the IR spectra of $\mathbf{2}-\mathbf{B N}^{2+}$ upon oxidation to $\mathbf{2}-\mathbf{B N}^{4+}$, measured in $\mathrm{CH}_{2} \mathrm{Cl}_{2} /{ }^{n} \mathrm{Bu}_{4} \mathrm{NPF}_{6}$

Figure S72. Deconvolutions of the Carbonyl bands of $\mathbf{2}-\mathbf{B N}^{2+}$ (left) and $\mathbf{2}-\mathbf{B N}^{4+}$ (right) 54

Figure S73. Changes in the UV/Vis/NIR spectra of $\mathbf{2}-\mathbf{B N}^{\mathbf{2 +}}$ upon oxidation to $\mathbf{2}-\mathbf{B N}^{4+}$ (left) and deconvolution (right), measured in $\mathrm{CH}_{2} \mathrm{Cl}_{2} /{ }^{n} \mathrm{Bu}_{4} \mathrm{NPF}_{6}$.

Figure S74. Changes in the UV/Vis/NIR spectra of $\mathbf{2}-\mathbf{B N}^{2+}$ upon oxidation to $\mathbf{2}-\mathbf{B} \mathbf{N}^{4+}$ (left) and deconvolution (right), measured in $\mathrm{CH}_{2} \mathrm{Cl}_{2} /{ }^{n} \mathrm{Bu}_{4} \mathrm{NPF}_{6}$ 55

Compound 2-NB: 56

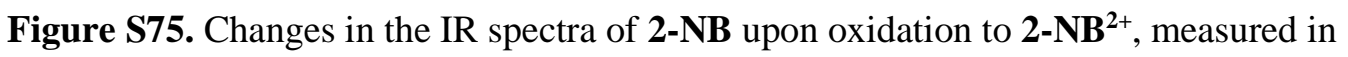
$\mathrm{CH}_{2} \mathrm{Cl}_{2} /{ }^{n} \mathrm{Bu}_{4} \mathrm{NPF}_{6}$ 56

Figure S76. Changes in the IR spectra of $\mathbf{2}-\mathbf{N B}^{2+}$ upon oxidation to $\mathbf{2}-\mathbf{N B}^{\mathbf{4 +}}$, measured in $\mathrm{CH}_{2} \mathrm{Cl}_{2} /{ }^{n} \mathrm{Bu}_{4} \mathrm{NPF}_{6}$. 56

Figure S77. Changes in the IR spectra of $\mathbf{2}-\mathbf{N B}^{4+}$ upon oxidation to $\mathbf{2}-\mathbf{N B}^{6+}$, measured in $\mathrm{CH}_{2} \mathrm{Cl}_{2} /{ }^{n} \mathrm{Bu}_{4} \mathrm{NPF}_{6}$ 56

Figure S78. Deconvolutions of the Carbonyl bands of $\mathbf{2 - \mathbf { N B } ^ { 2 + }}$ (left) and 2-NB ${ }^{4+}$ (right) 57

Figure S79. Changes in the UV/Vis/NIR spectra of 2-NB upon oxidation to $2-\mathbf{N B}^{2+}$ (left) and deconvolution (right), measured in $\mathrm{CH}_{2} \mathrm{Cl}_{2} /{ }^{n} \mathrm{Bu}_{4} \mathrm{NPF}_{6}$.

Figure S80. Changes in the UV/Vis/NIR spectra of $\mathbf{2}-\mathbf{N B}^{2+}$ upon oxidation to $\mathbf{2}-\mathbf{N B}^{\mathbf{4 +}}$ (left) and deconvolution (right), measured in $\mathrm{CH}_{2} \mathrm{Cl}_{2} /{ }^{n} \mathrm{Bu}_{4} \mathrm{NPF}_{6}$.

Figure S81. Changes in the UV/Vis/NIR spectra of $\mathbf{2}-\mathbf{N B}^{4+}$ upon oxidation to $\mathbf{2}-\mathbf{N B}^{\mathbf{6 +}}$ (left) and deconvolution (right), measured in $\mathrm{CH}_{2} \mathrm{Cl}_{2} /{ }^{n} \mathrm{Bu}_{4} \mathrm{NPF}_{6}$ 58 


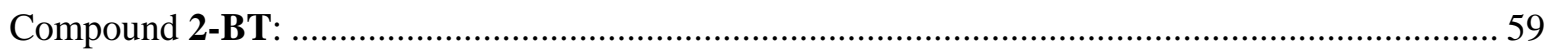

Figure S82. EPR spectra of 2-BT ${ }^{2+}$ (left) and 2-BT ${ }^{4+}$ (right) at room temperature...................... 59

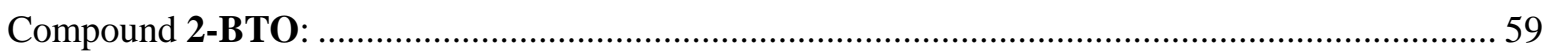

Figure S83. EPR spectra of 2-BTO ${ }^{2+}$ (left) and 2-BTO ${ }^{4+}$ (right) at room temperature. ............... 59

Compound 2-BTE:

Figure S84. EPR spectra of 2-BTE ${ }^{2+}$ (left) and 2-BTE ${ }^{4+}$ (right) at room temperature. ................ 59

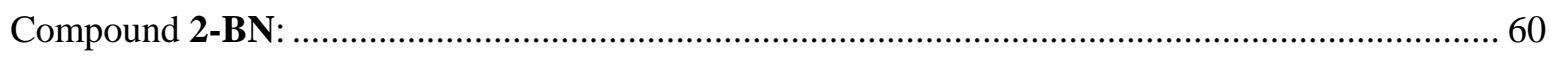

Figure S85. EPR spectra of 2-BN ${ }^{2+}$ (left) and 2-BN ${ }^{2+}$ (right) at room temperature. ...................... 60

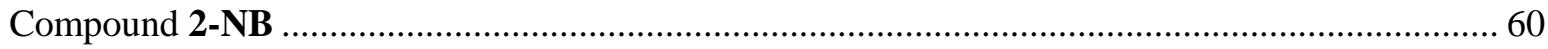

Figure S86. EPR spectra of 2-NB ${ }^{2+}$ (left) and 2-NB ${ }^{4+}$ (right) at room temperature. ....................... 60

Figure S87. EPR spectra of 2-NB ${ }^{6+}$ (left) and of thianthrenium hexafluoroantimonate (right) at room temperature.

Figure S88. Carbonyl bands of $\mathbf{2}-\mathbf{N B}^{4+}$ (left) and $\mathbf{2}-\mathbf{N B}^{\mathbf{6 +}}$ (right) generated via chemical oxidation with the appropriate number of equivalents of thianthrenium hexafluoroantimonate in $\mathrm{CH}_{2} \mathrm{Cl}_{2} . .61$ 


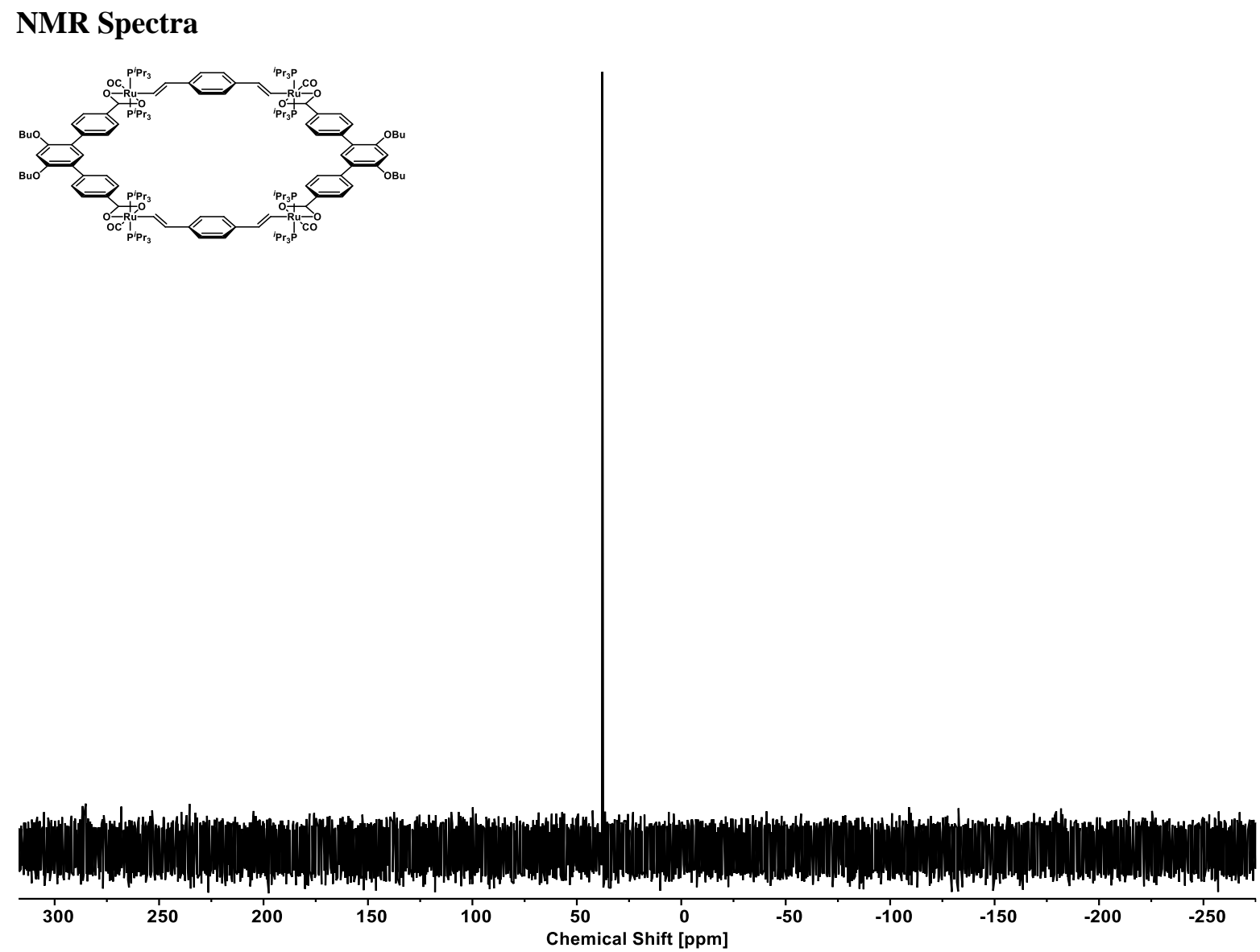

Figure S1. ${ }^{31} \mathrm{P}\left\{{ }^{1} \mathrm{H}\right\}$ NMR spectrum of macrocycle 2-BT.
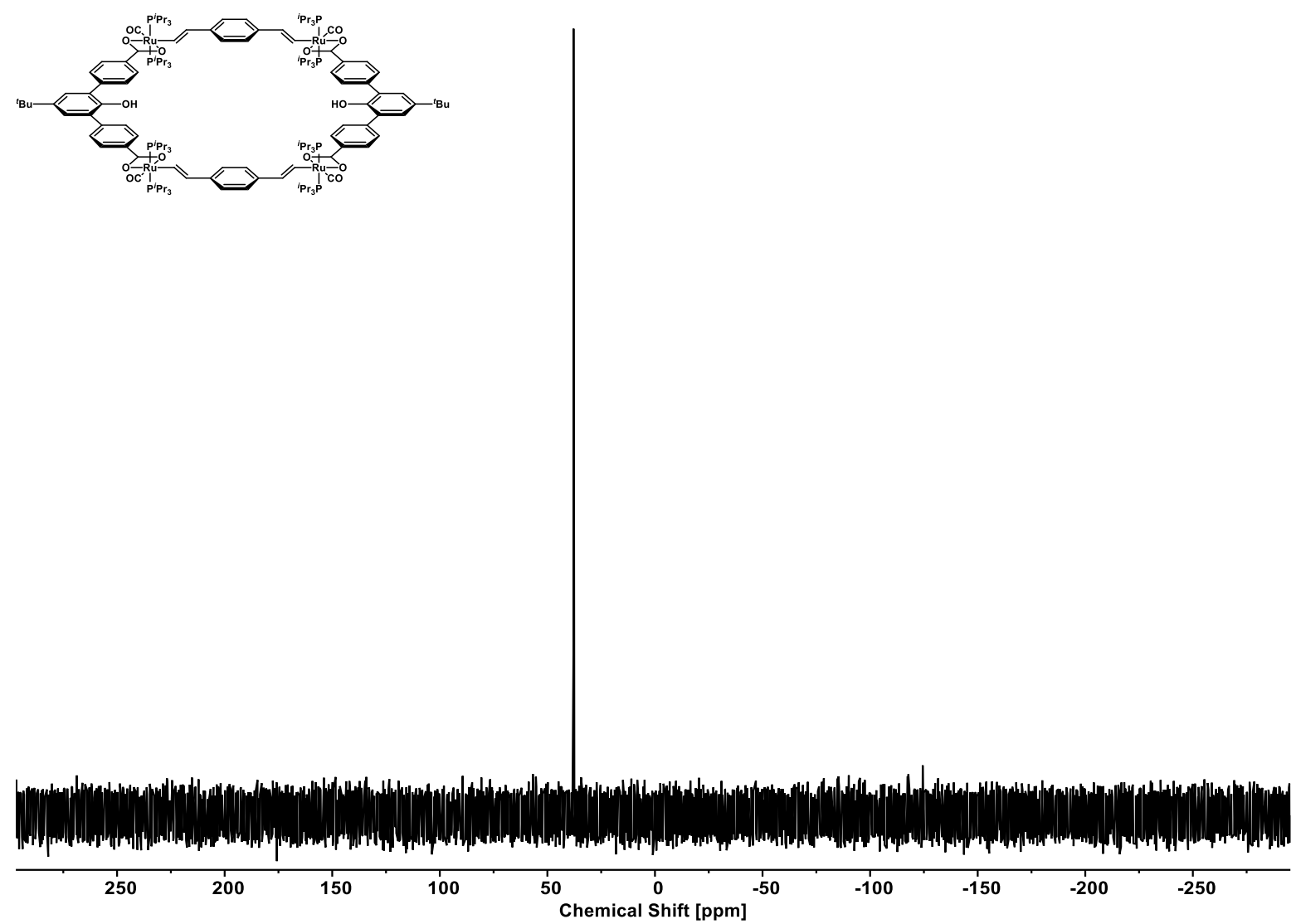

Figure S2. ${ }^{31} \mathrm{P}\left\{{ }^{1} \mathrm{H}\right\}$ NMR spectrum of macrocycle 2-BTO. 

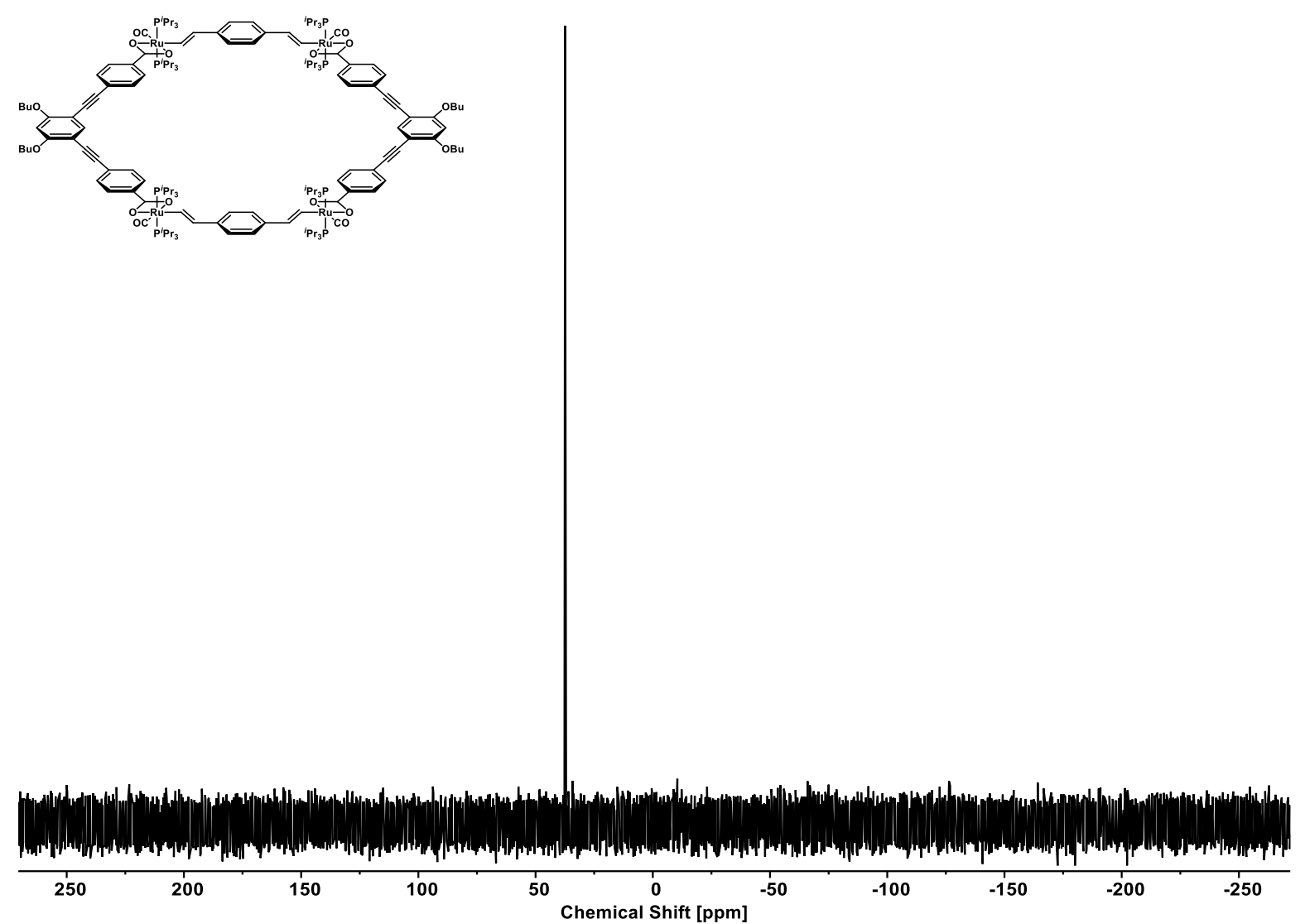

Figure S3. ${ }^{31} \mathrm{P}\left\{{ }^{1} \mathrm{H}\right\}$ NMR spectrum of macrocycle 2-BTE.
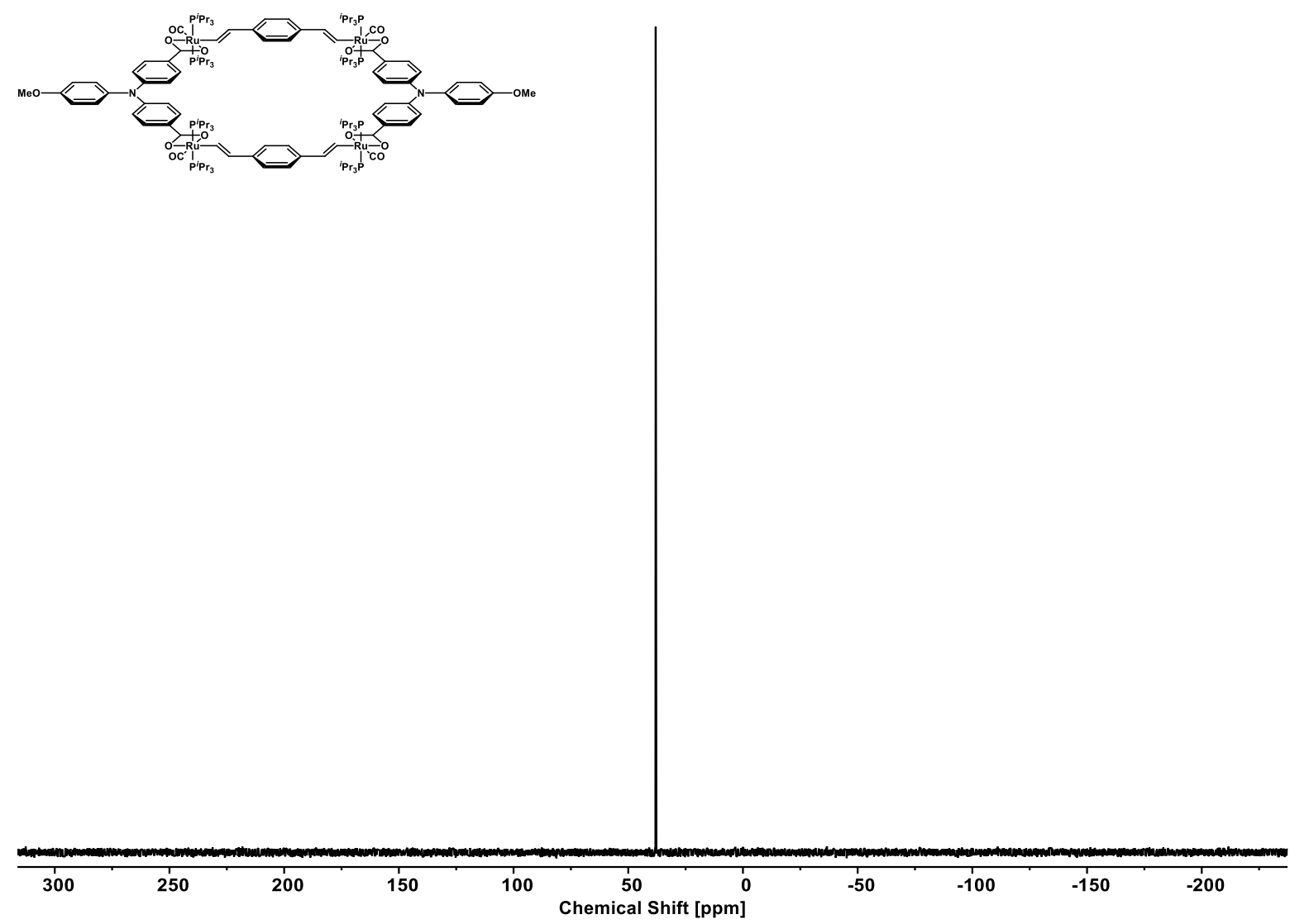

Figure S4. ${ }^{31} \mathrm{P}\left\{{ }^{1} \mathrm{H}\right\}$ NMR spectrum of macrocycle 2-BN. 


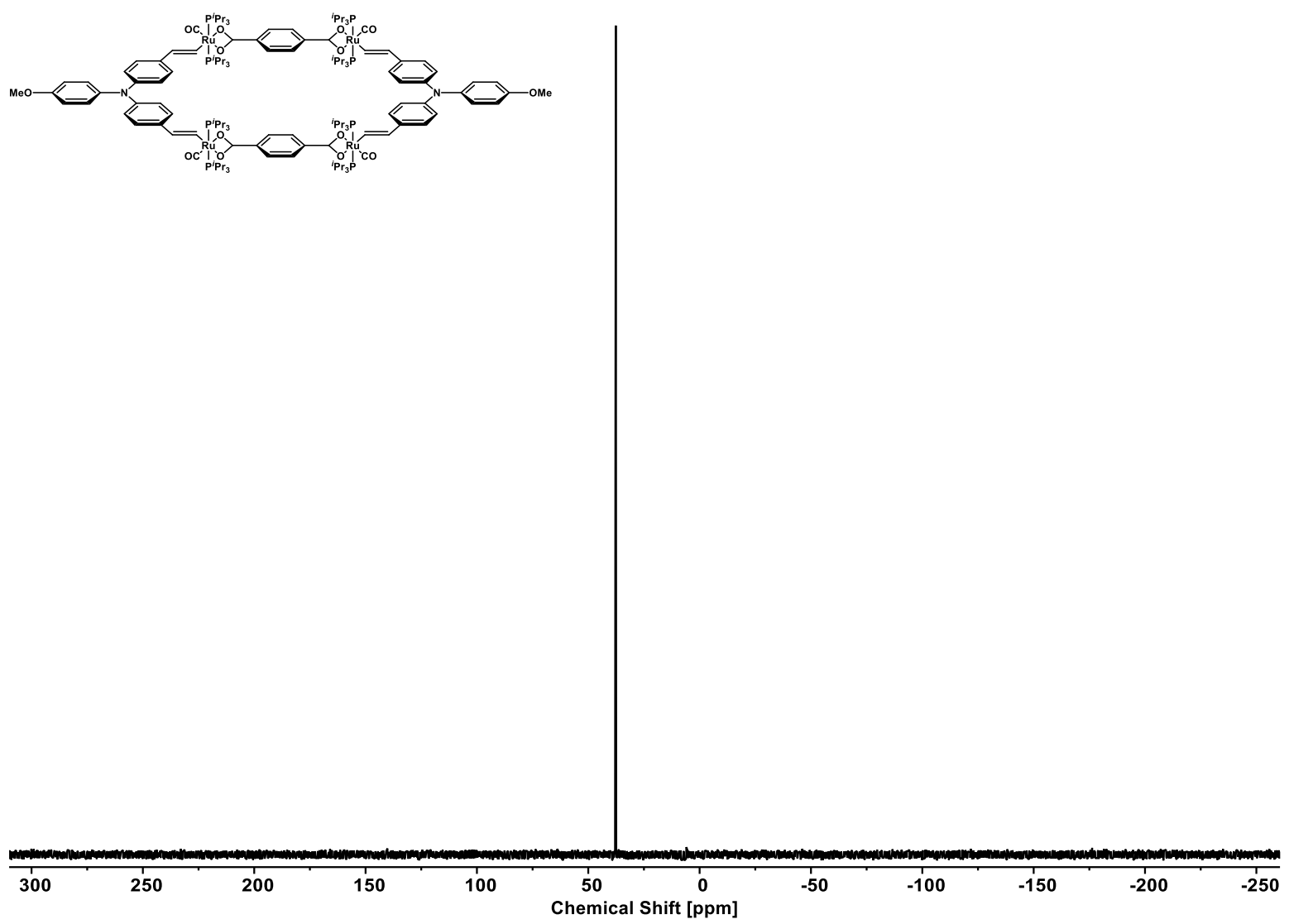

Figure S5. ${ }^{31} \mathrm{P}\left\{{ }^{1} \mathrm{H}\right\}$ NMR spectrum of macrocycle 2-NB.
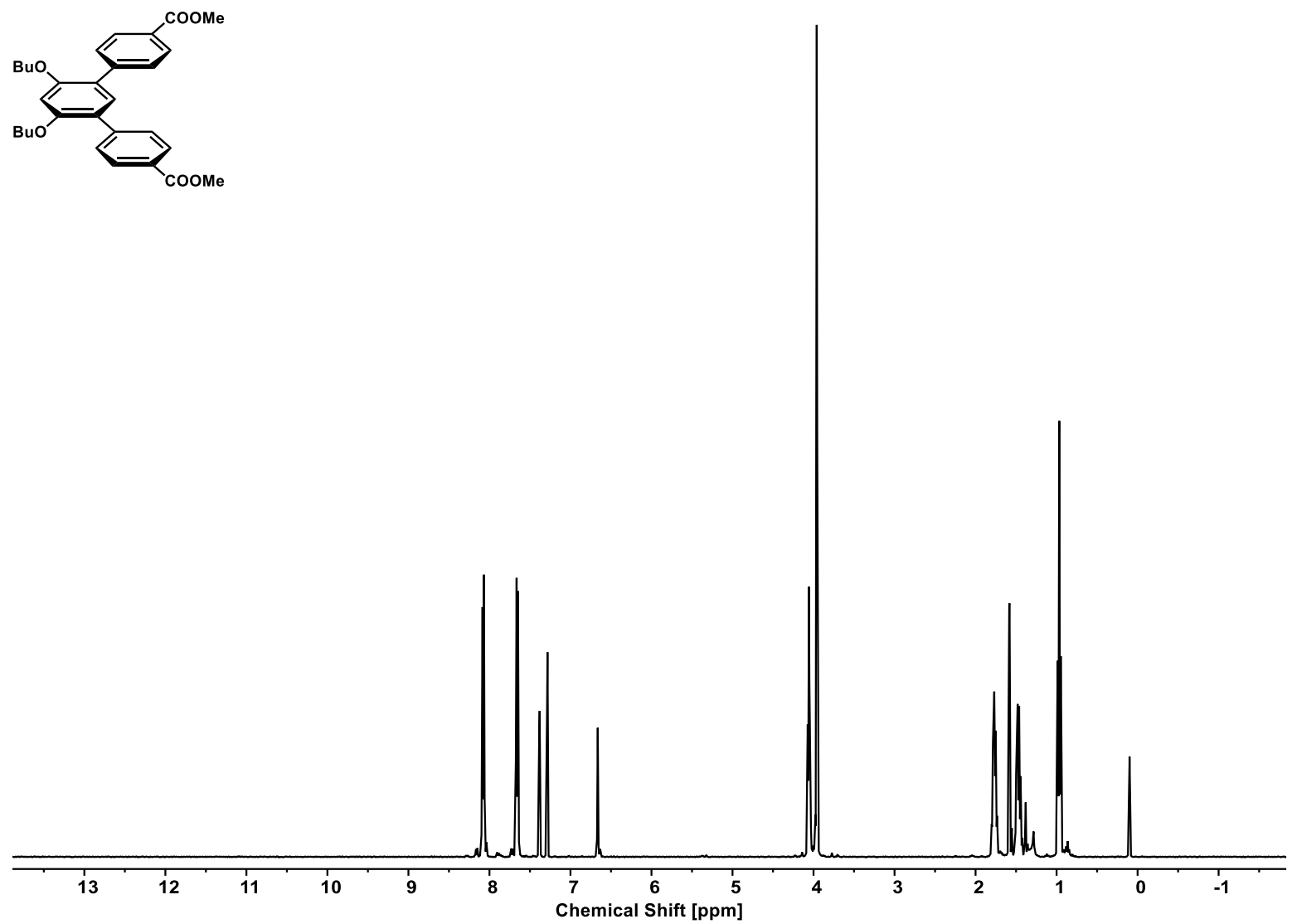

Figure S6. ${ }^{1} \mathrm{H}$ NMR spectrum of organic ligand $\mathbf{L}-\mathbf{T}_{\text {Me. }}$.

S10 

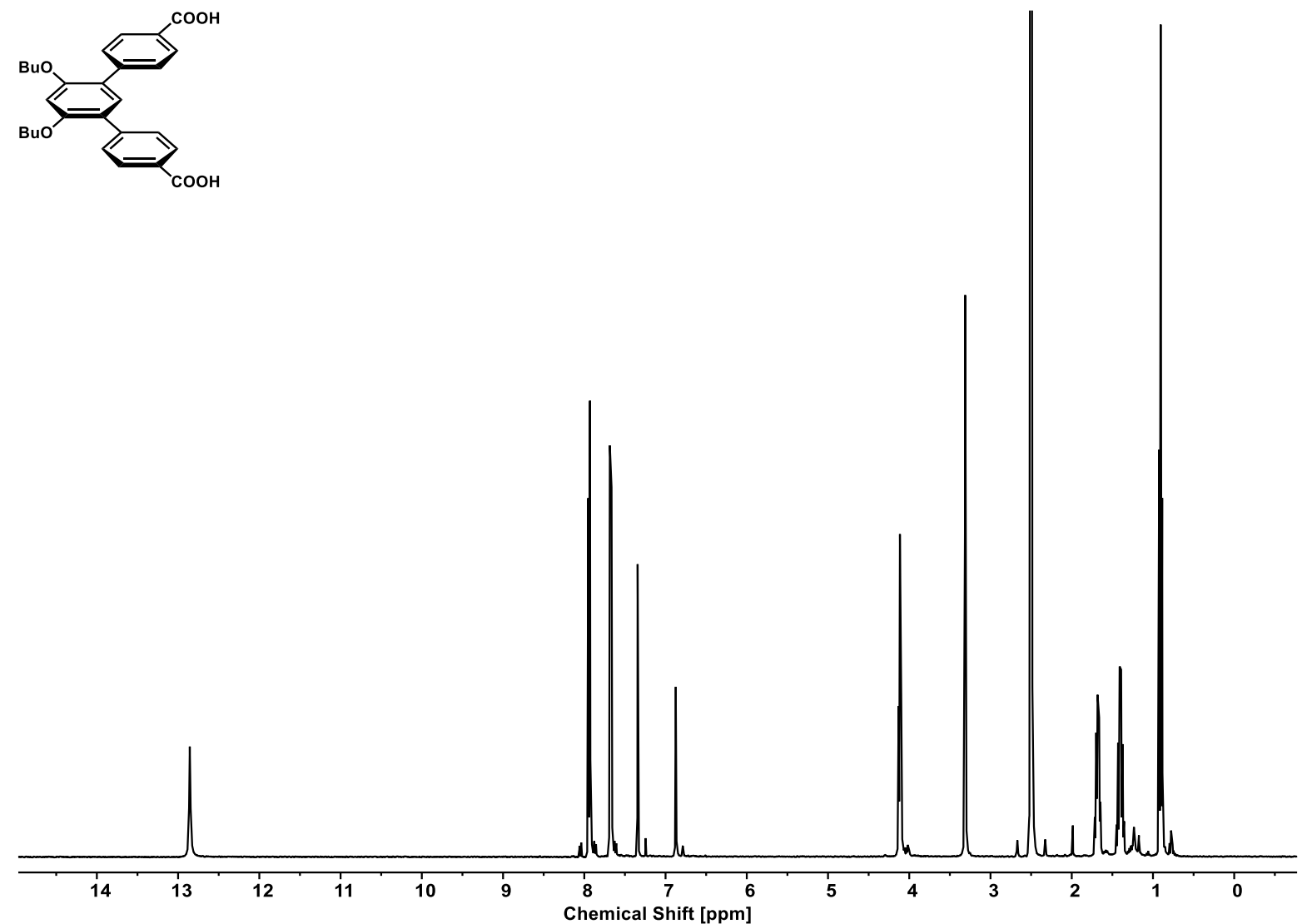

Figure S7. ${ }^{1} \mathrm{H}$ NMR spectrum of organic ligand $\mathbf{L}-\mathbf{T}_{\mathbf{H}}$.
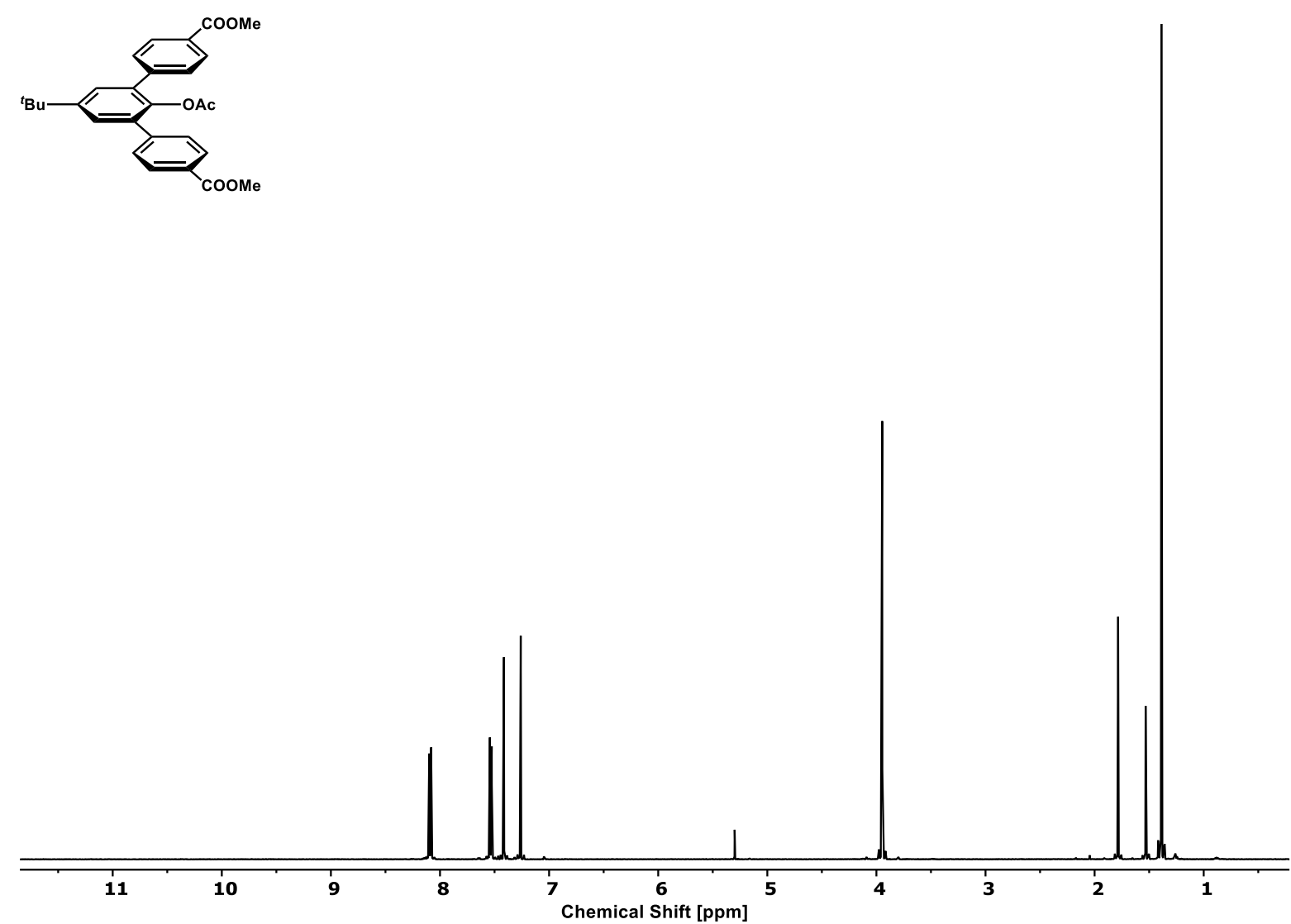

Figure S8. ${ }^{1} \mathrm{H}$ NMR spectrum of organic ligand $\mathbf{L}$-TO $\mathbf{O}_{\mathbf{M e}}$. 

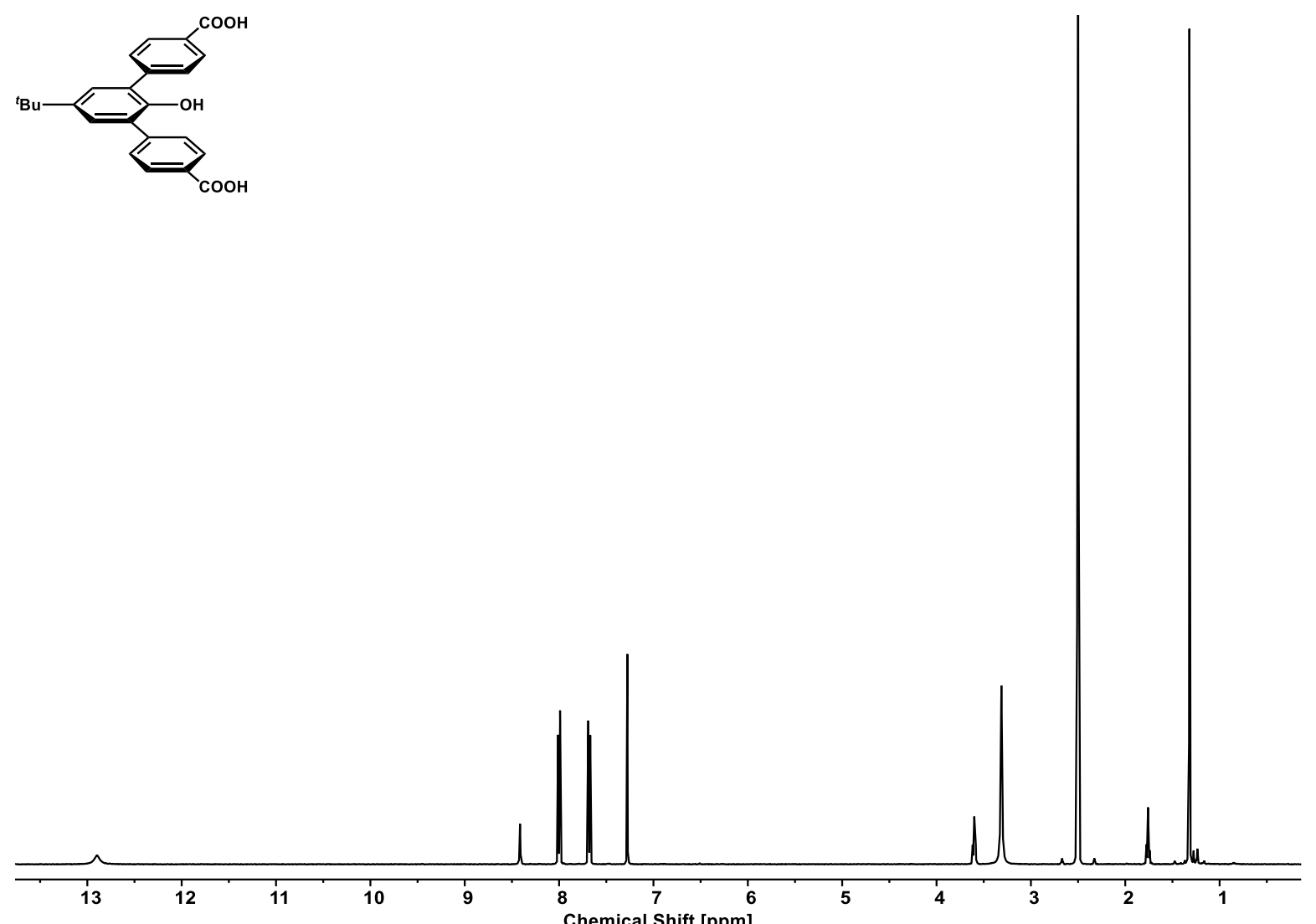

Figure S9. ${ }^{1} \mathrm{H}$ NMR spectrum of organic ligand $\mathbf{L}-\mathbf{T O}_{\mathbf{H}}$.
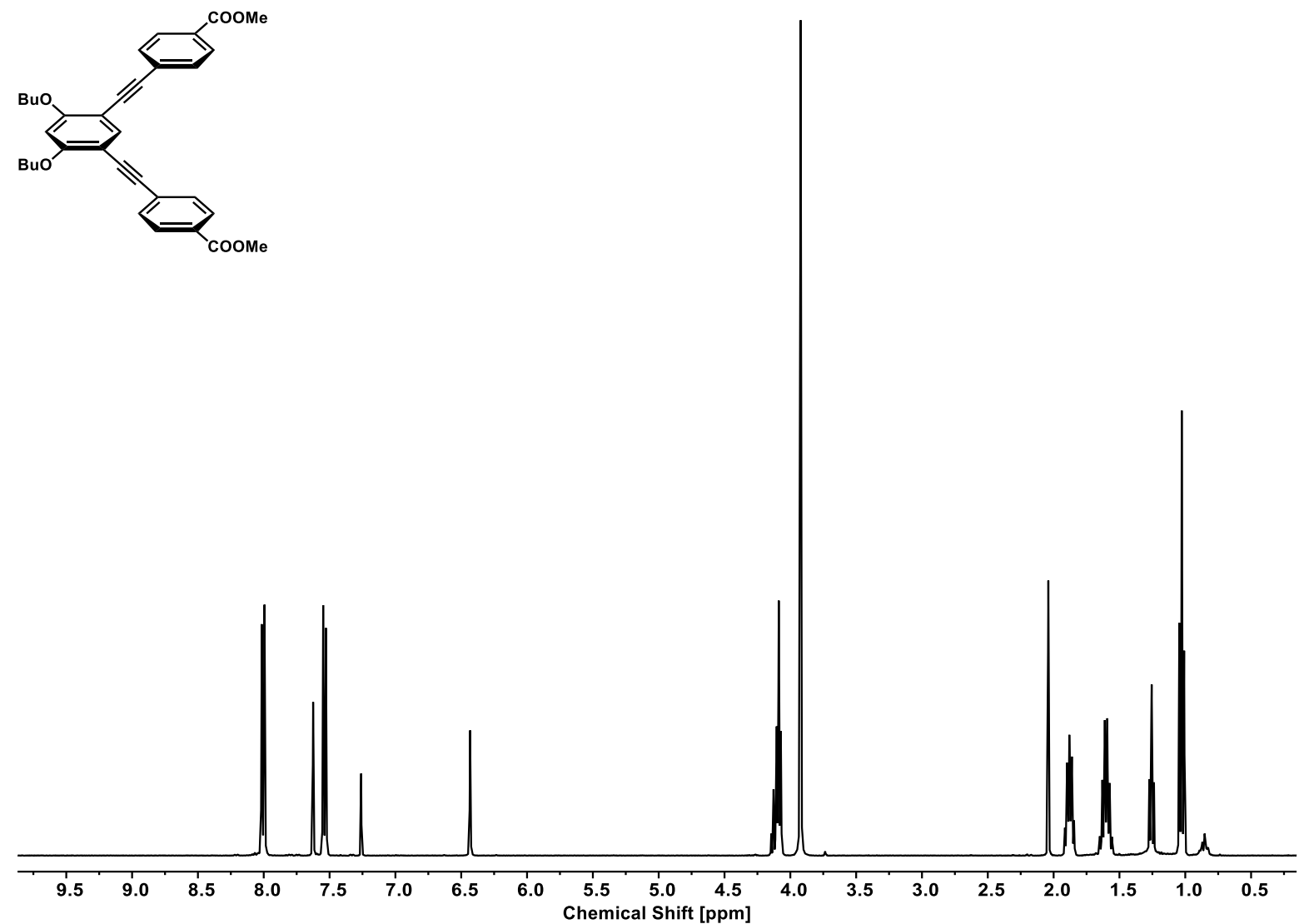

Figure S10. ${ }^{1} \mathrm{H}$ NMR spectrum of organic ligand $\mathbf{L}-\mathbf{T E}_{\text {Me. }}$. 

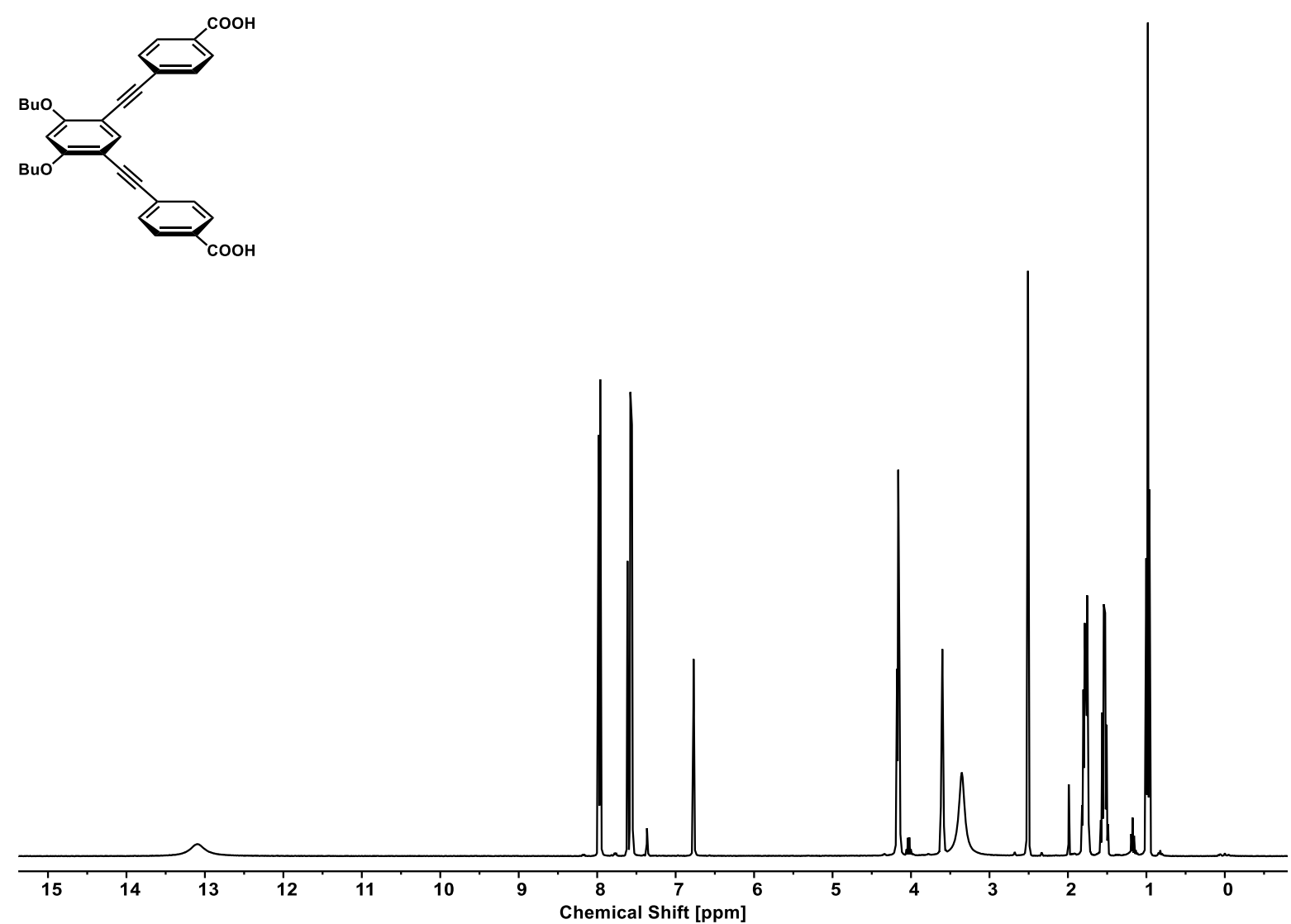

Figure S11. ${ }^{1} \mathrm{H}$ NMR spectrum of organic ligand $\mathbf{L}-\mathbf{T E}_{\mathbf{H}}$.
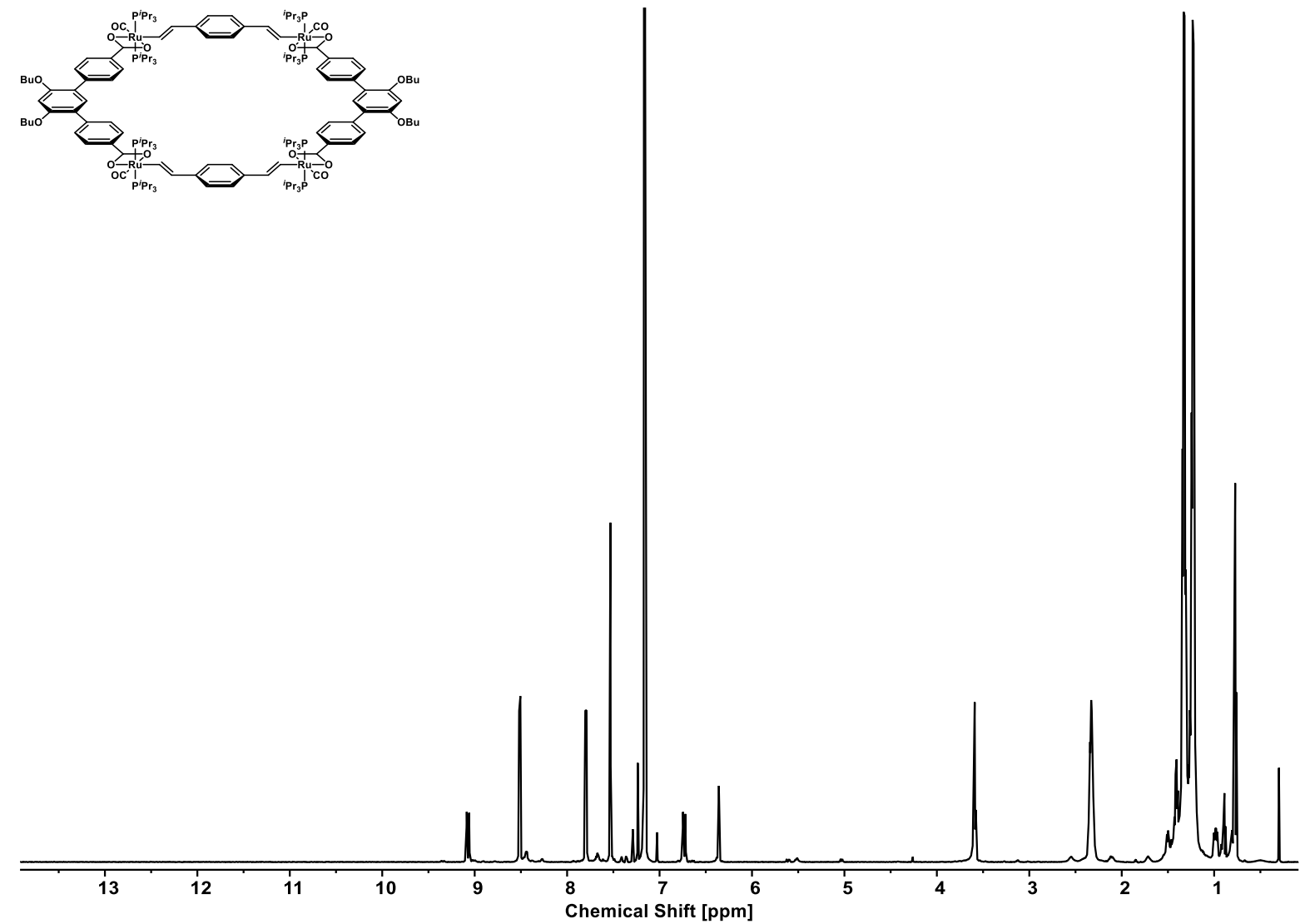

Figure S12. ${ }^{1} \mathrm{H}$ NMR spectrum of macrocycle 2-BT. 


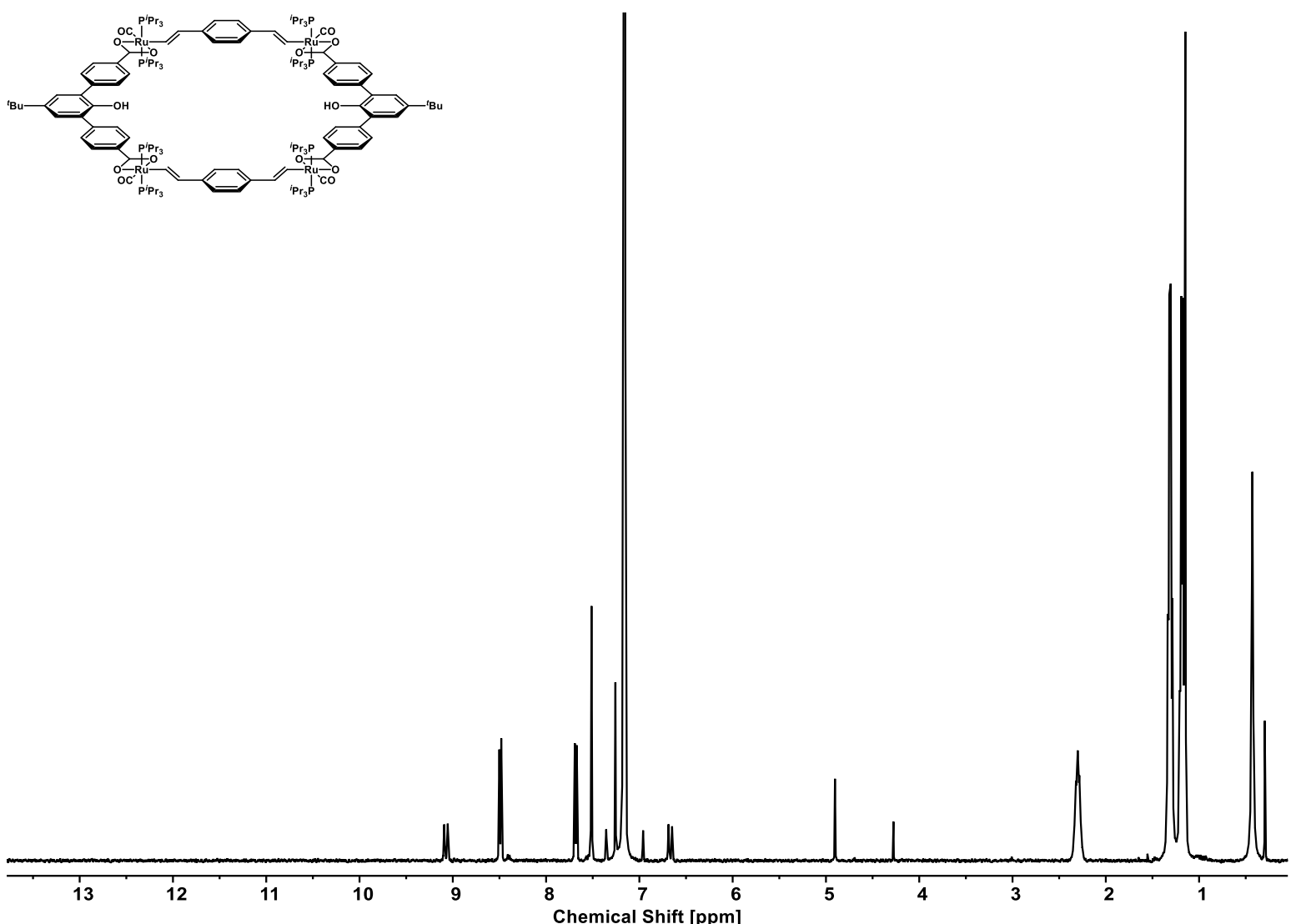

Figure S13. ${ }^{1} \mathrm{H}$ NMR spectrum of macrocycle 2-BTO.

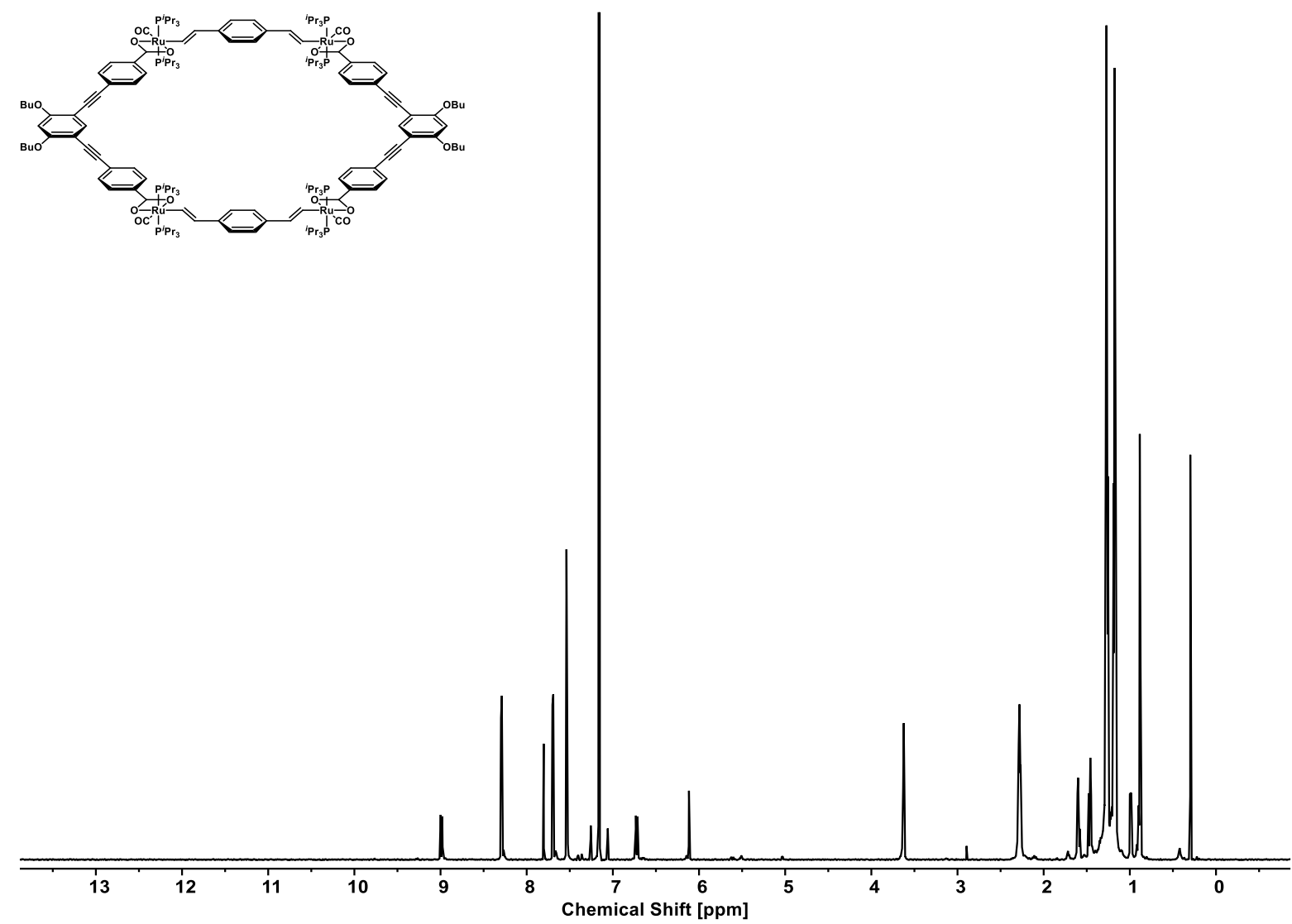

Figure S14. ${ }^{1} \mathrm{H}$ NMR spectrum of macrocycle 2-BTE. 


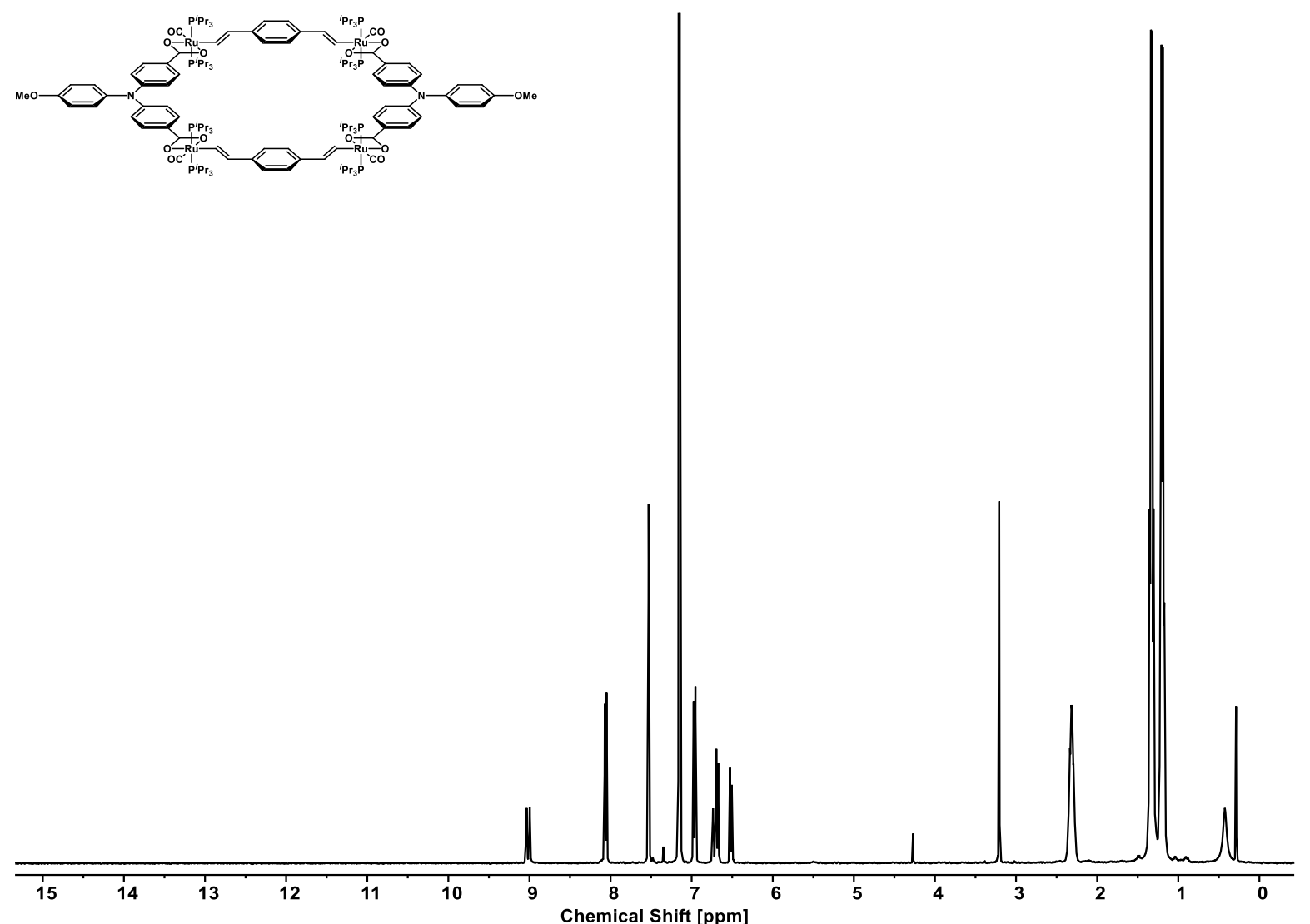

Figure S15. ${ }^{1} \mathrm{H}$ NMR spectrum of macrocycle 2-BN.

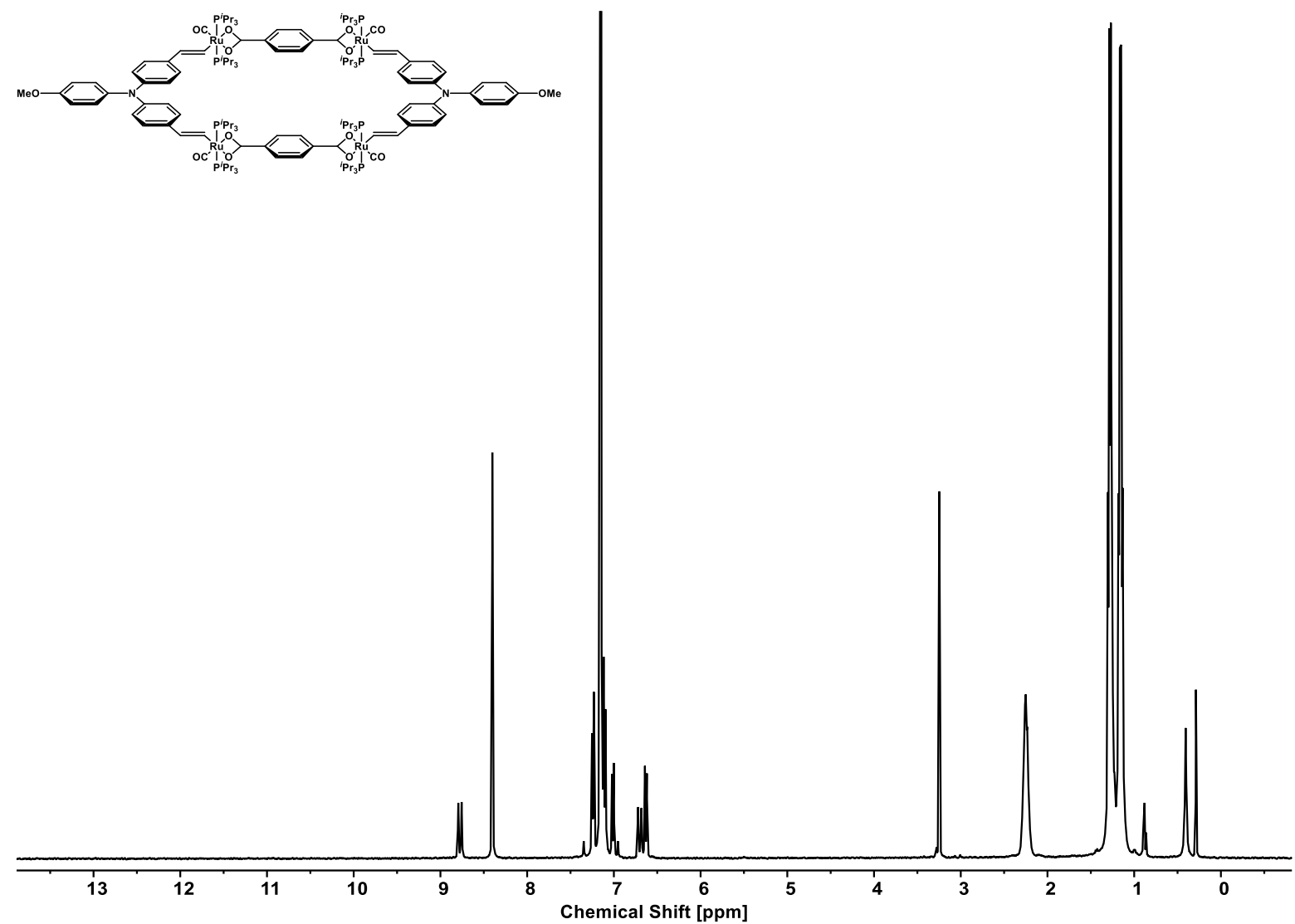

Figure S16. ${ }^{1} \mathrm{H}$ NMR spectrum of macrocycle 2-NB. 

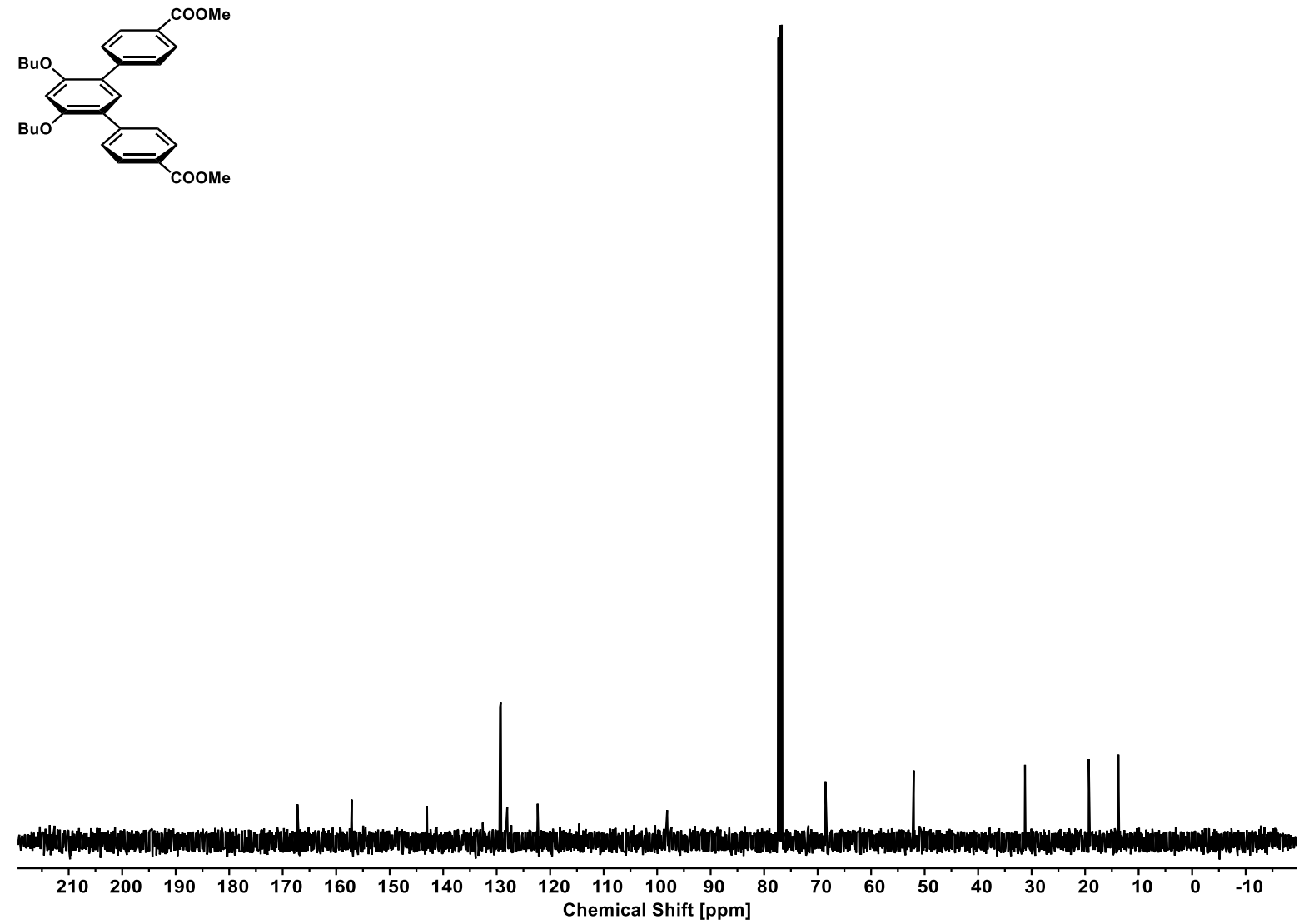

Figure S17. ${ }^{13} \mathrm{C}\left\{{ }^{1} \mathrm{H}\right\}$ NMR spectrum of organic ligand $\mathbf{L}-\mathbf{T}_{\text {Me. }}$.
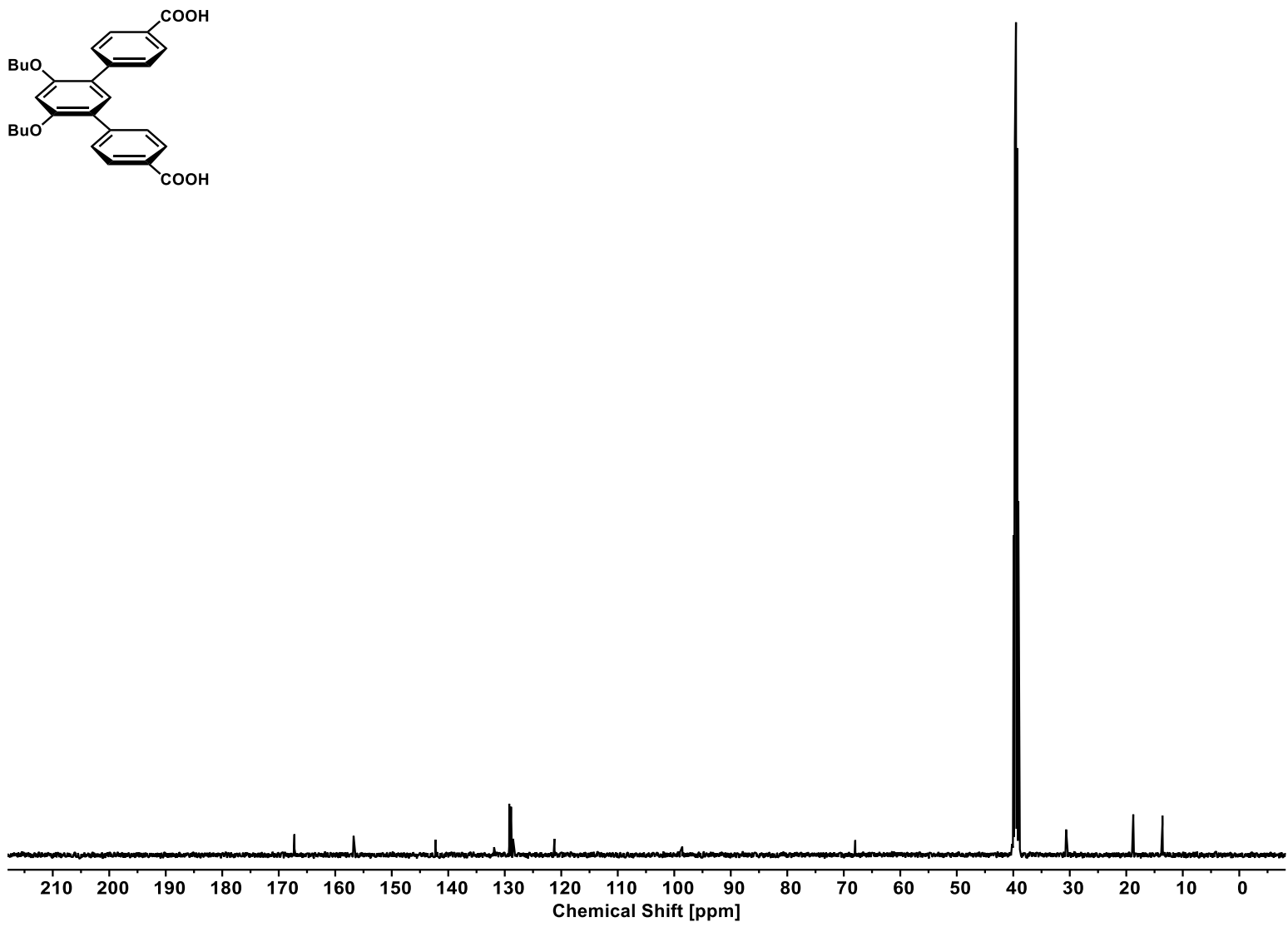

Figure S18. ${ }^{13} \mathrm{C}\left\{{ }^{1} \mathrm{H}\right\}$ NMR spectrum of organic ligand $\mathbf{L}-\mathbf{T}_{\mathbf{H}}$ 

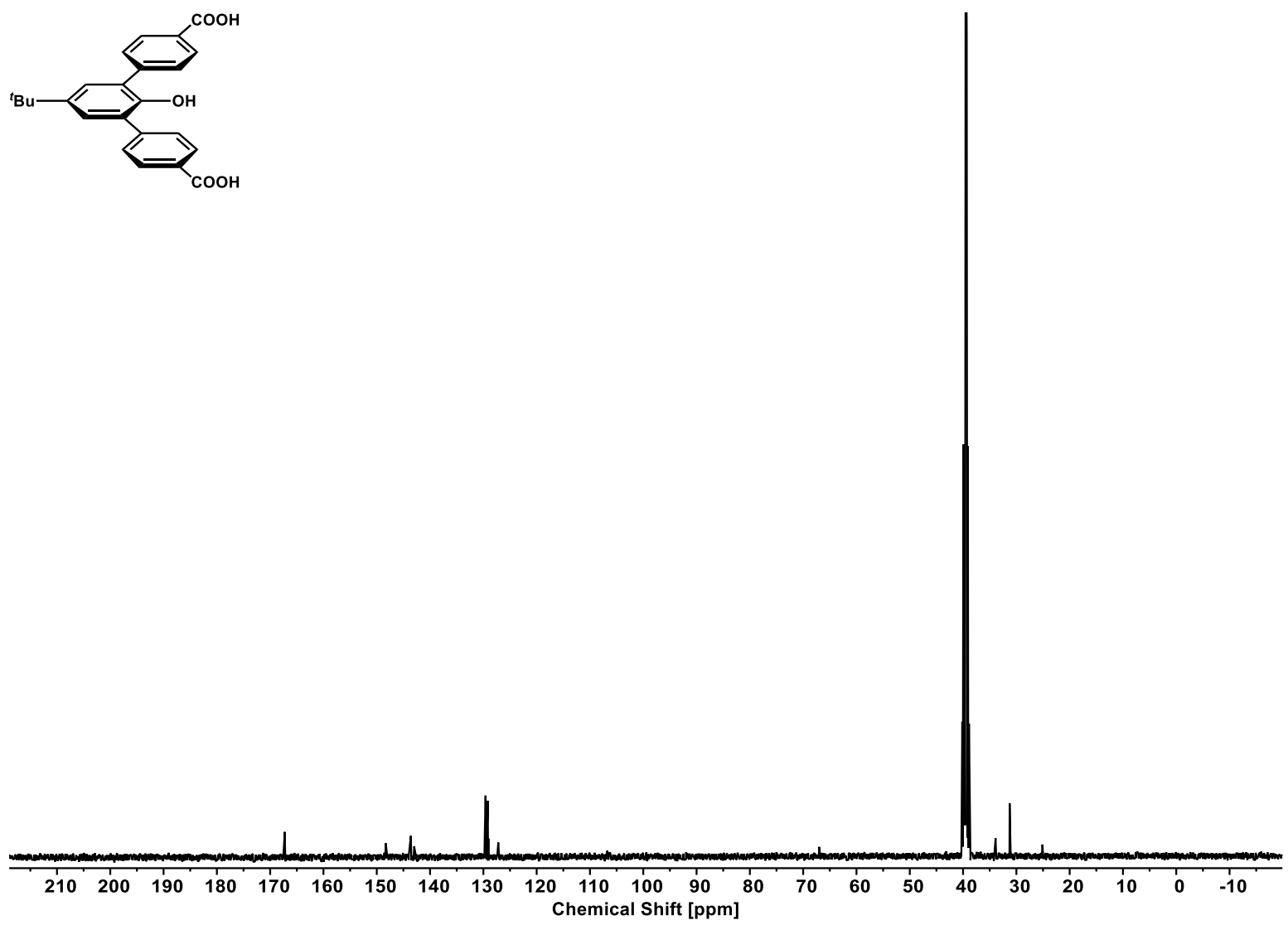

Figure S19. ${ }^{13} \mathrm{C}\left\{{ }^{1} \mathrm{H}\right\}$ NMR spectrum of organic ligand $\mathbf{L}-\mathbf{T} \mathbf{O}_{\mathbf{H}}$.
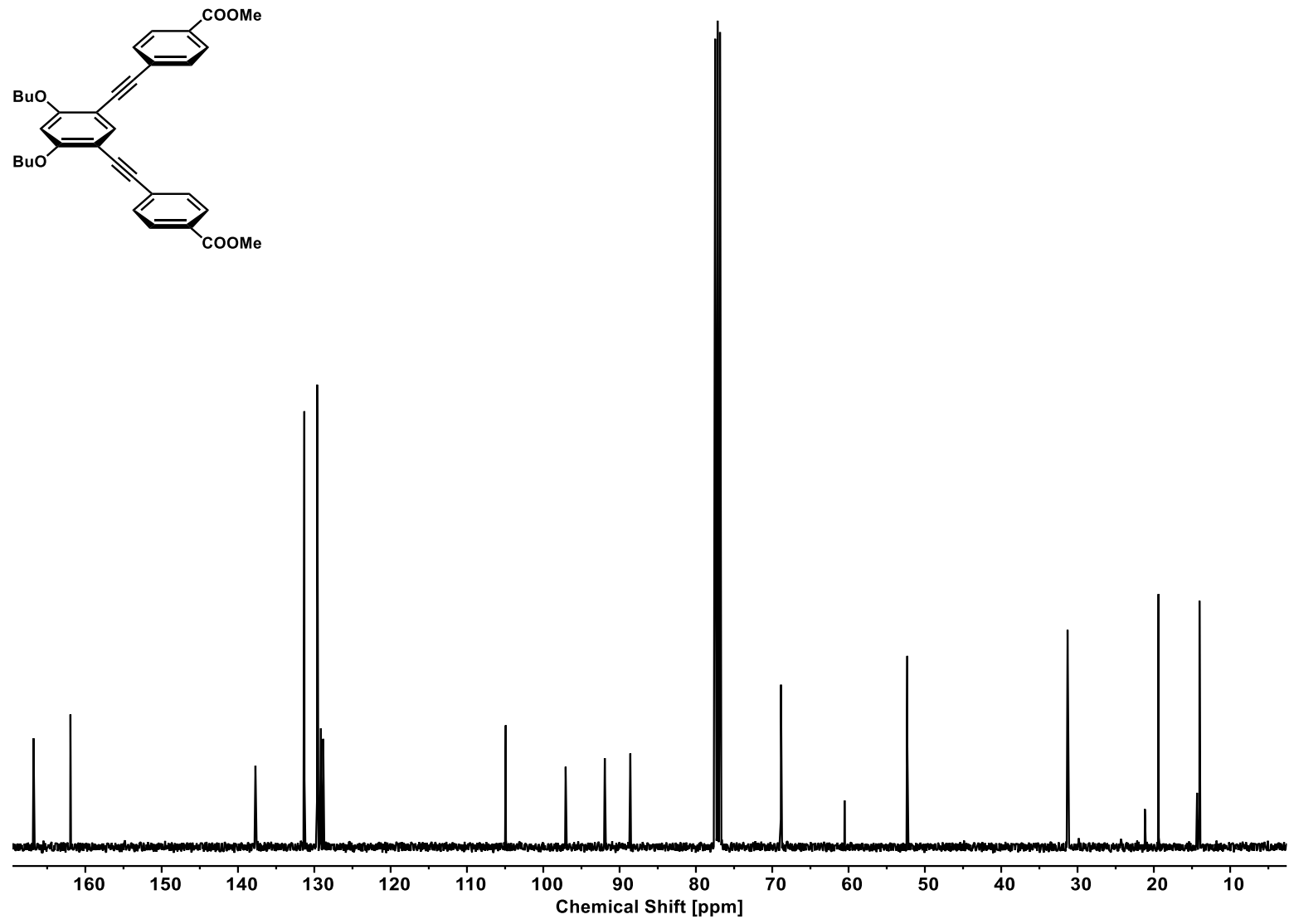

Figure S20. ${ }^{13} \mathrm{C}\left\{{ }^{1} \mathrm{H}\right\}$ NMR spectrum of organic ligand $\mathbf{L}-\mathbf{T E}_{\mathrm{Me}}$.

S17 

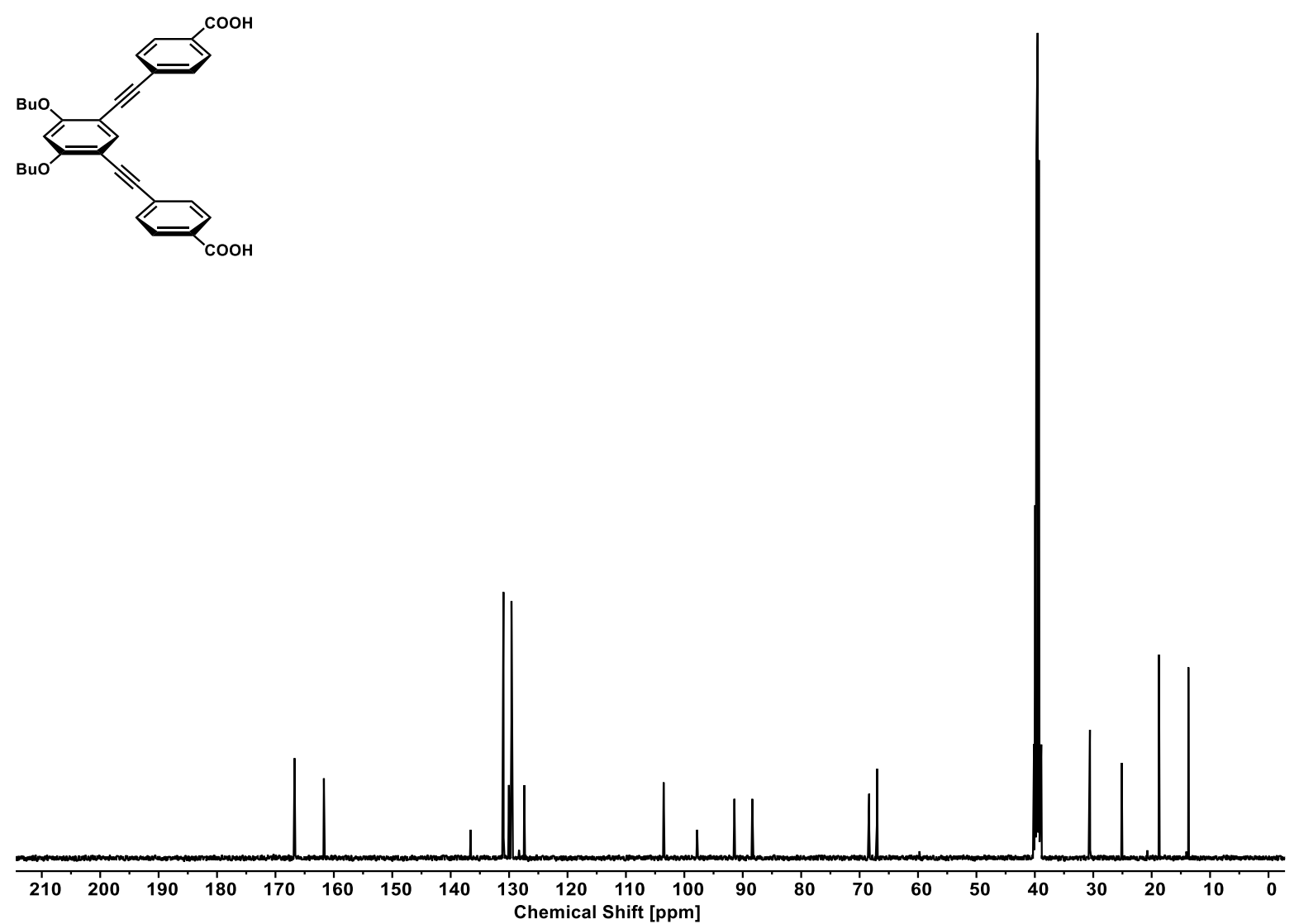

Figure S21. ${ }^{13} \mathrm{C}\left\{{ }^{1} \mathrm{H}\right\}$ NMR spectrum of organic ligand $\mathbf{L}-\mathbf{T E}_{\mathbf{H}}$.
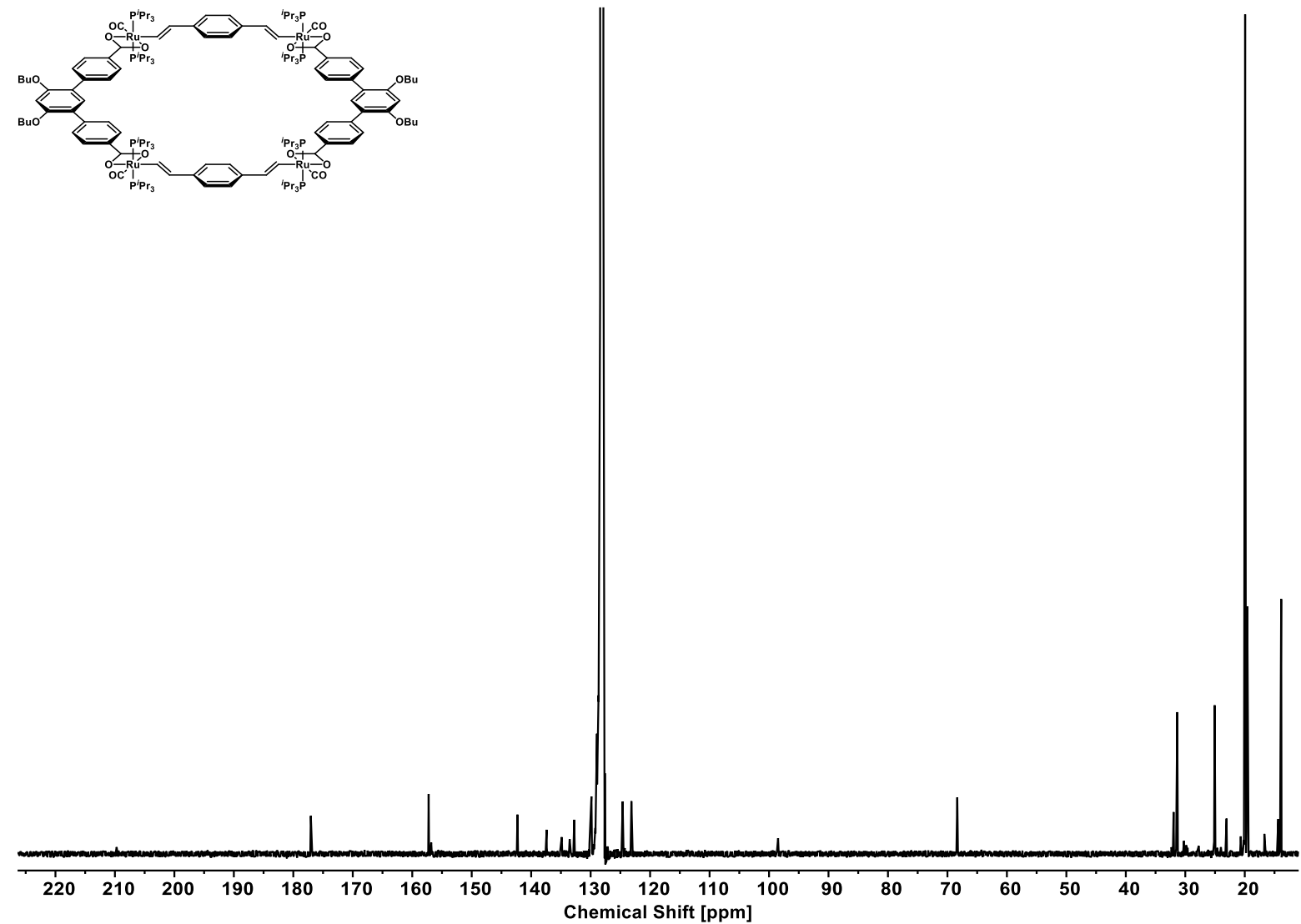

Figure S22. ${ }^{13} \mathrm{C}\left\{{ }^{1} \mathrm{H}\right\}$ NMR spectrum of macrocycle 2-BT. 


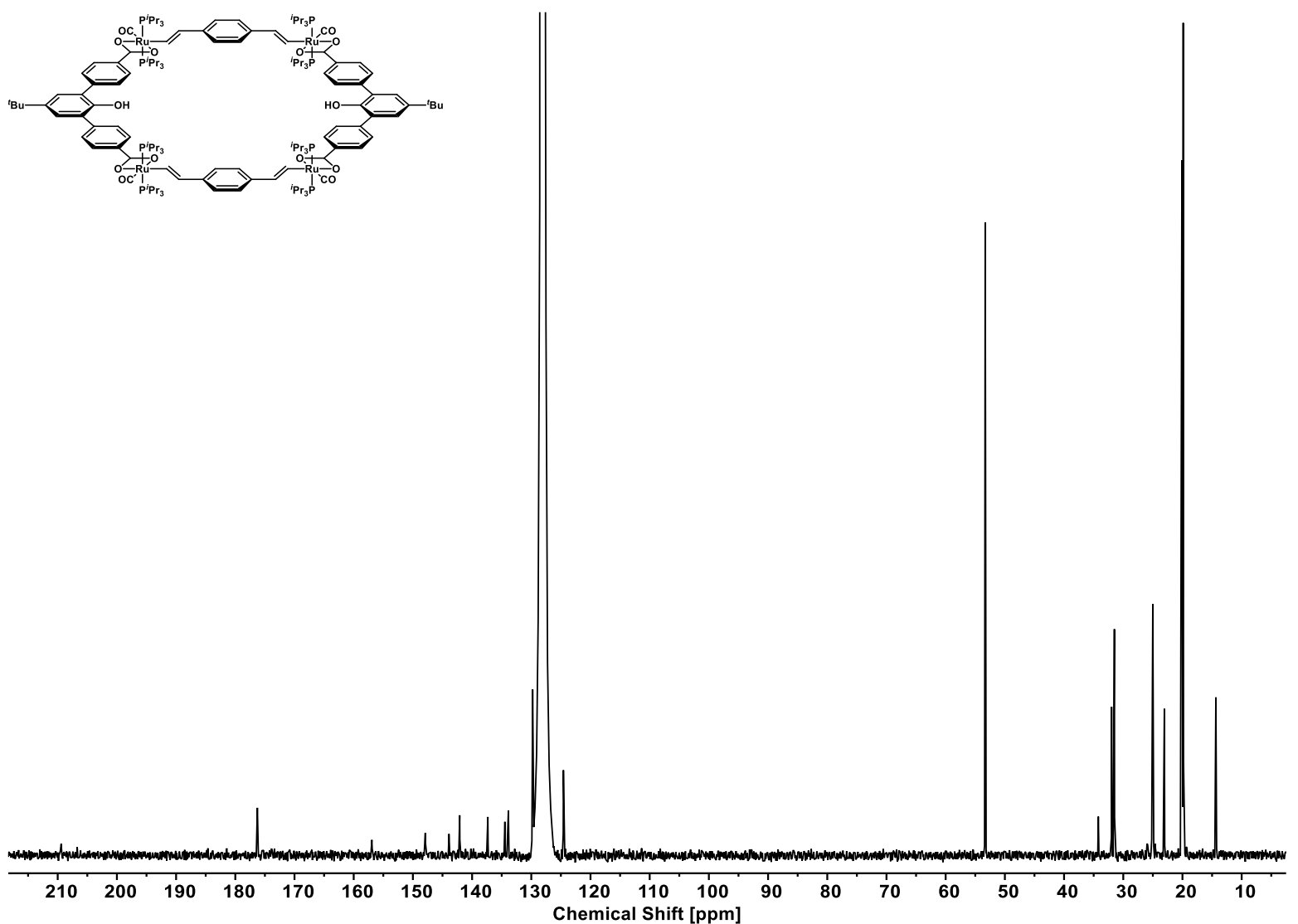

Figure S23. ${ }^{13} \mathrm{C}\left\{{ }^{1} \mathrm{H}\right\}$ NMR spectrum of macrocycle 2-BTO.

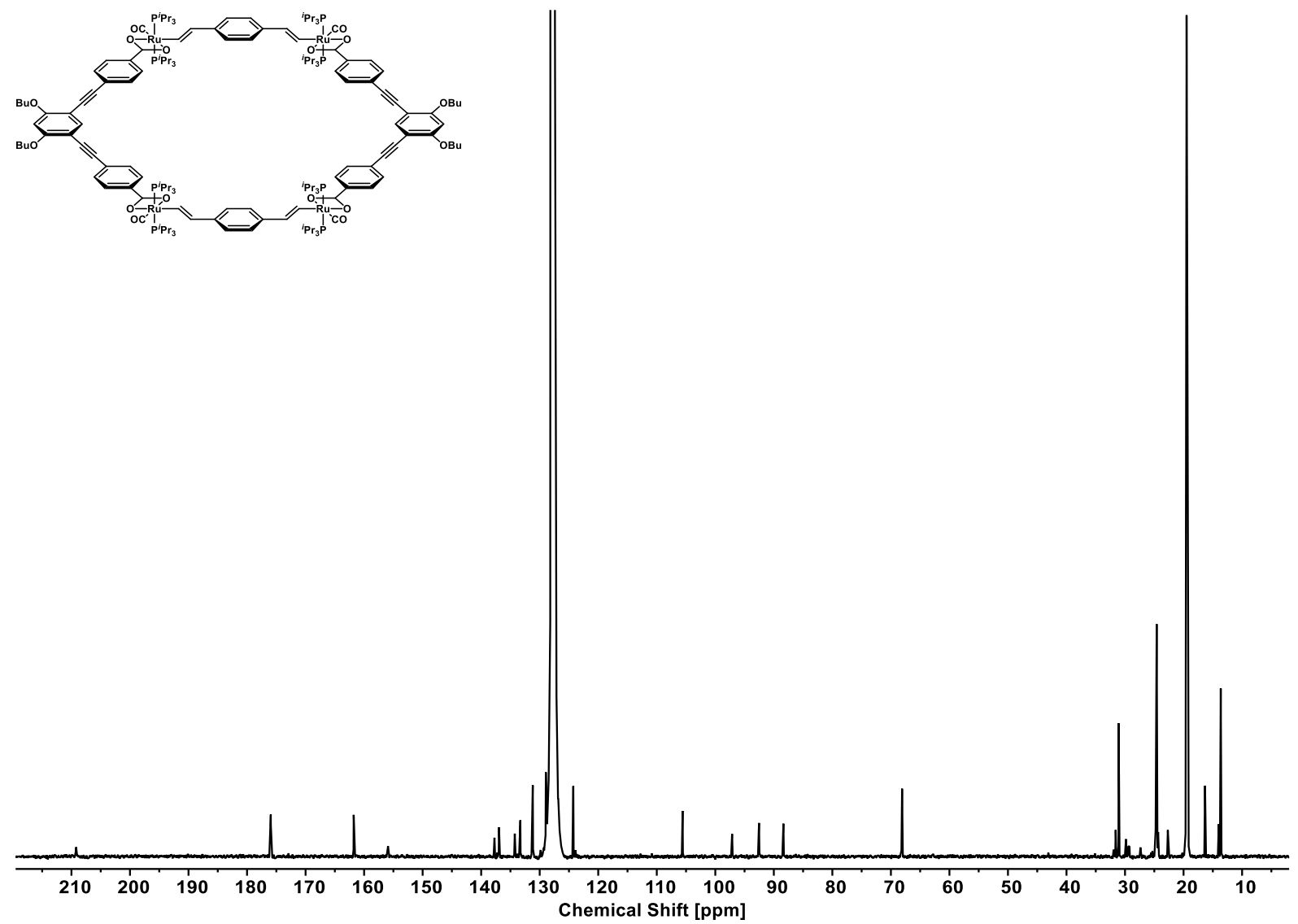

Figure S24. ${ }^{13} \mathrm{C}\left\{{ }^{1} \mathrm{H}\right\}$ NMR spectrum of macrocycle 2-BTE. 


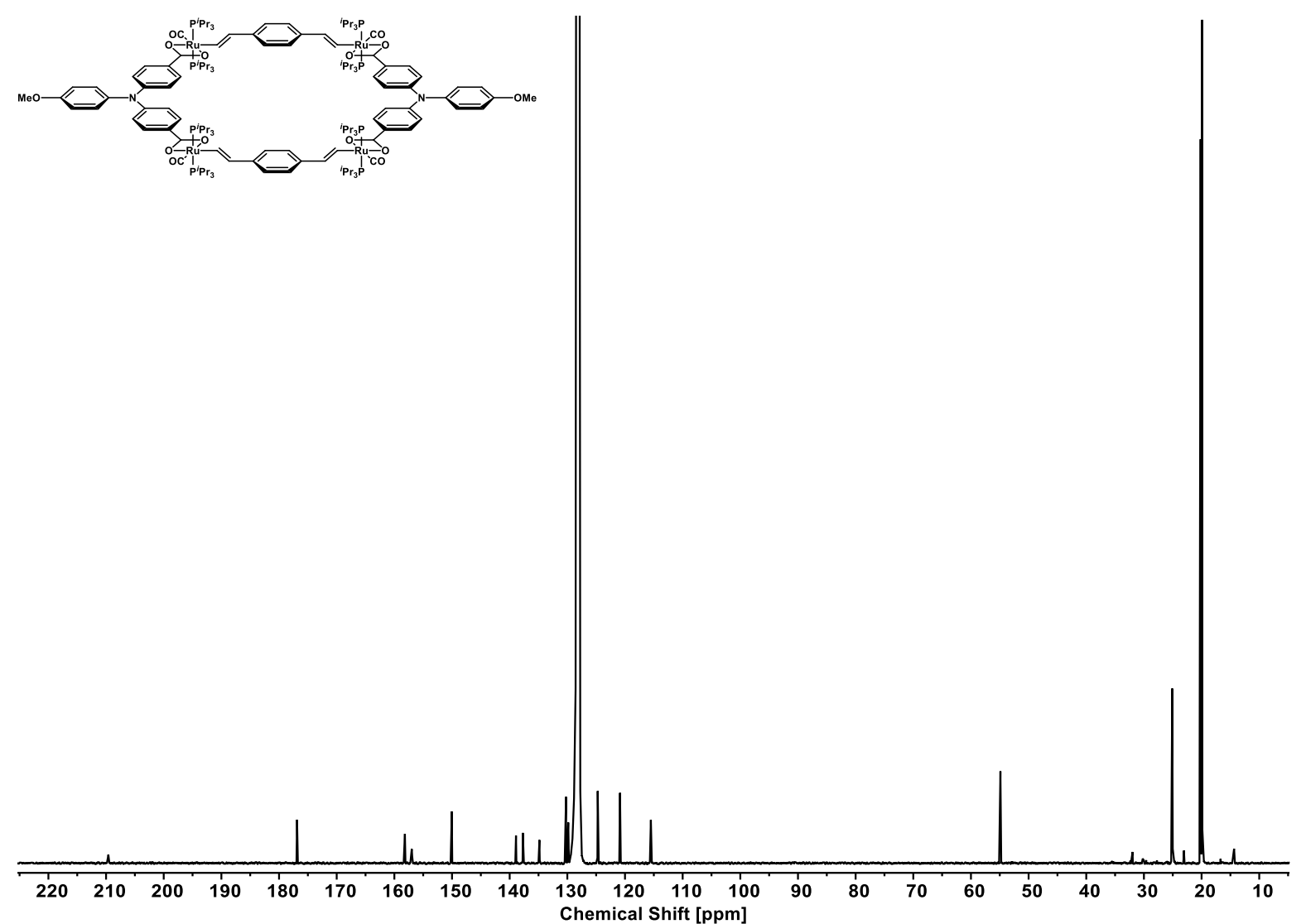

Figure S25. ${ }^{13} \mathrm{C}\left\{{ }^{1} \mathrm{H}\right\}$ NMR spectrum of macrocycle 2-BN.
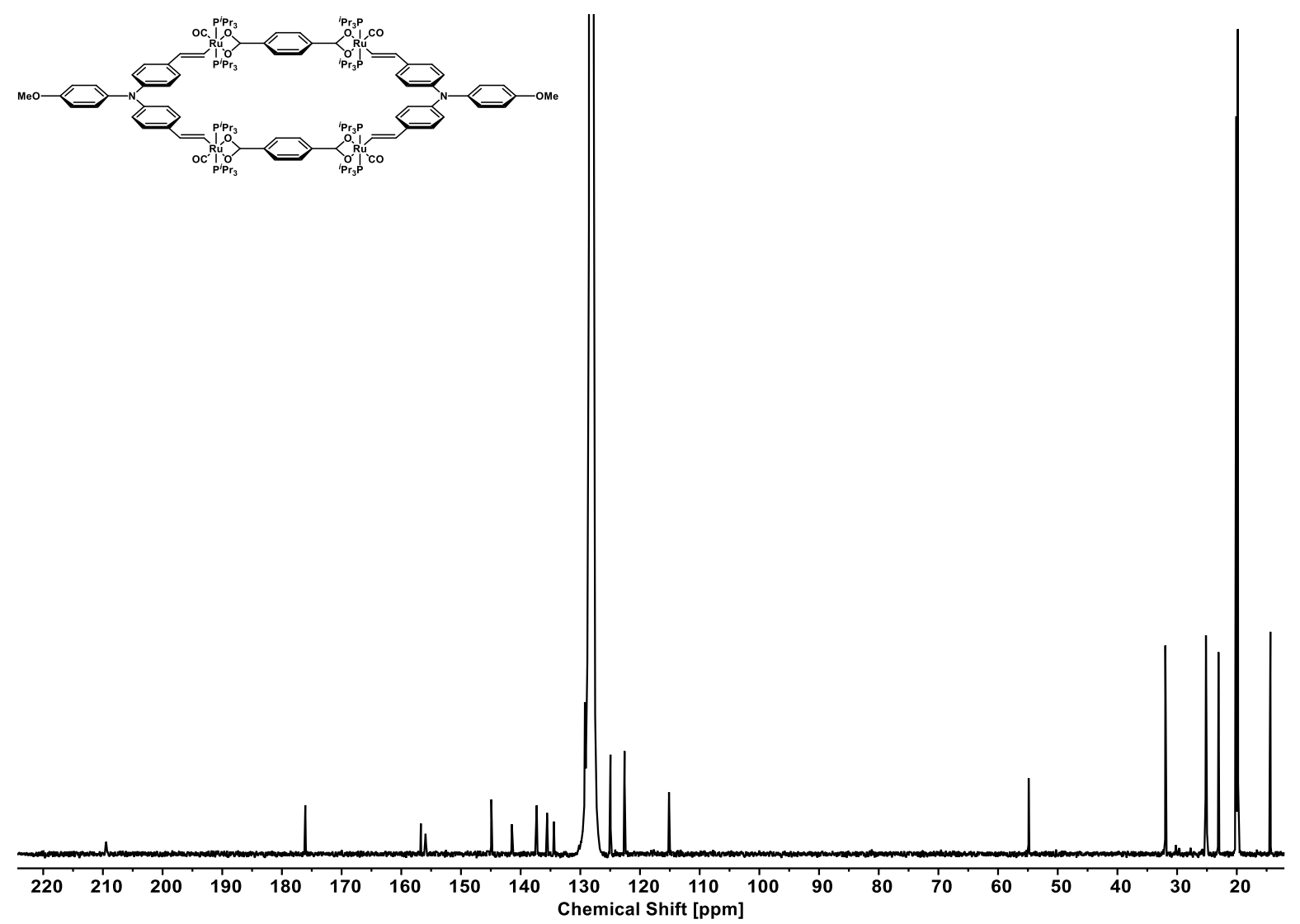

Figure S26. ${ }^{13} \mathrm{C}\left\{{ }^{1} \mathrm{H}\right\}$ NMR spectrum of macrocycle 2-NB. 

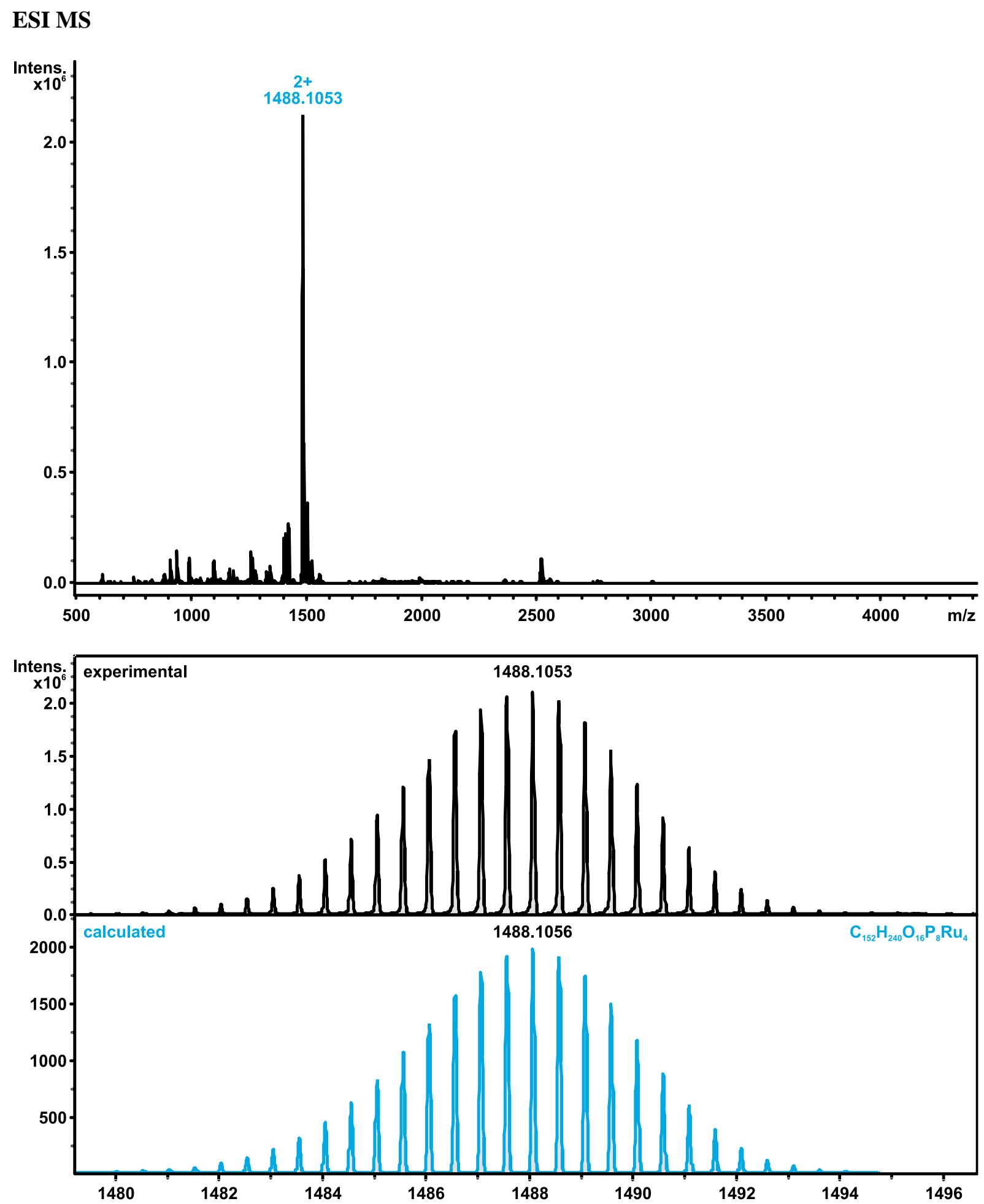

Figure S27. ESI Mass spectrum of macrocycle 2-BT (top); magnification of $[\mathrm{M}]^{2+}$ peak with calculated isotopic pattern (bottom). 

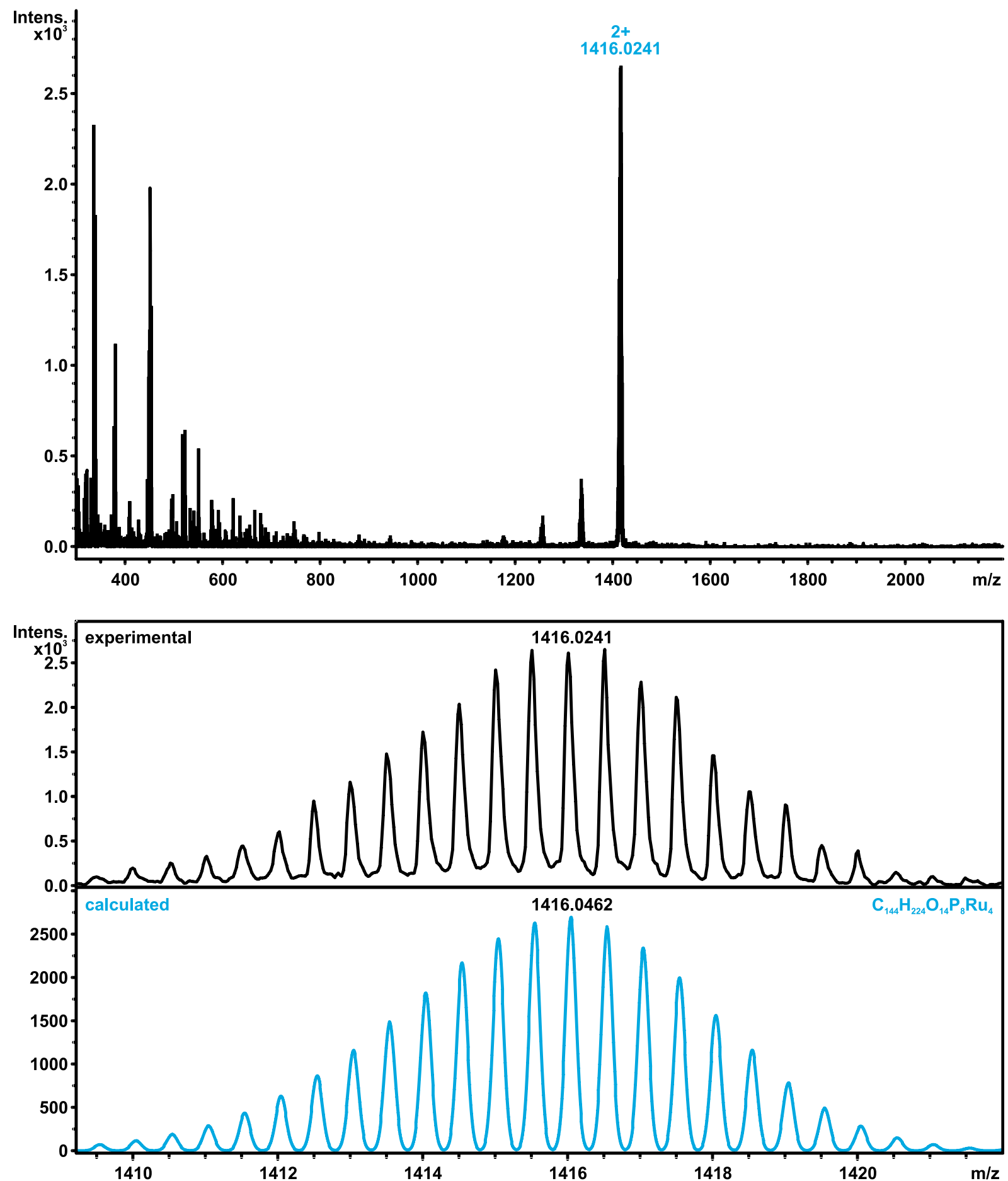

Figure S28. ESI Mass spectrum of macrocycle 2-BTO (top), magnification of $[\mathrm{M}]^{2+}$ peak with calculated isotopic pattern (bottom). 

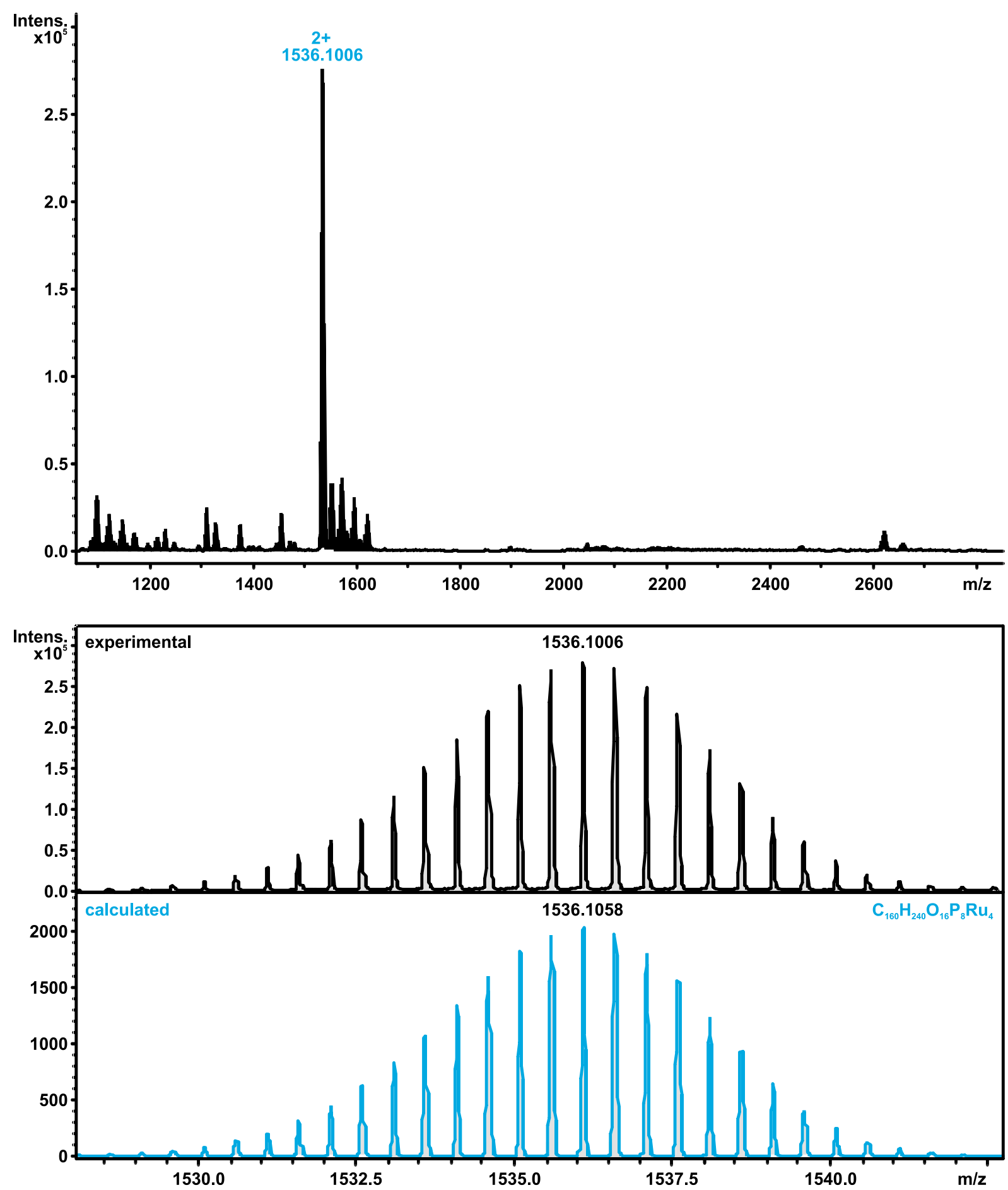

Figure S29. ESI Mass spectrum of macrocycle 2-BTE (top), magnification of $[\mathrm{M}]^{2+}$ peak with calculated isotopic pattern (bottom). 

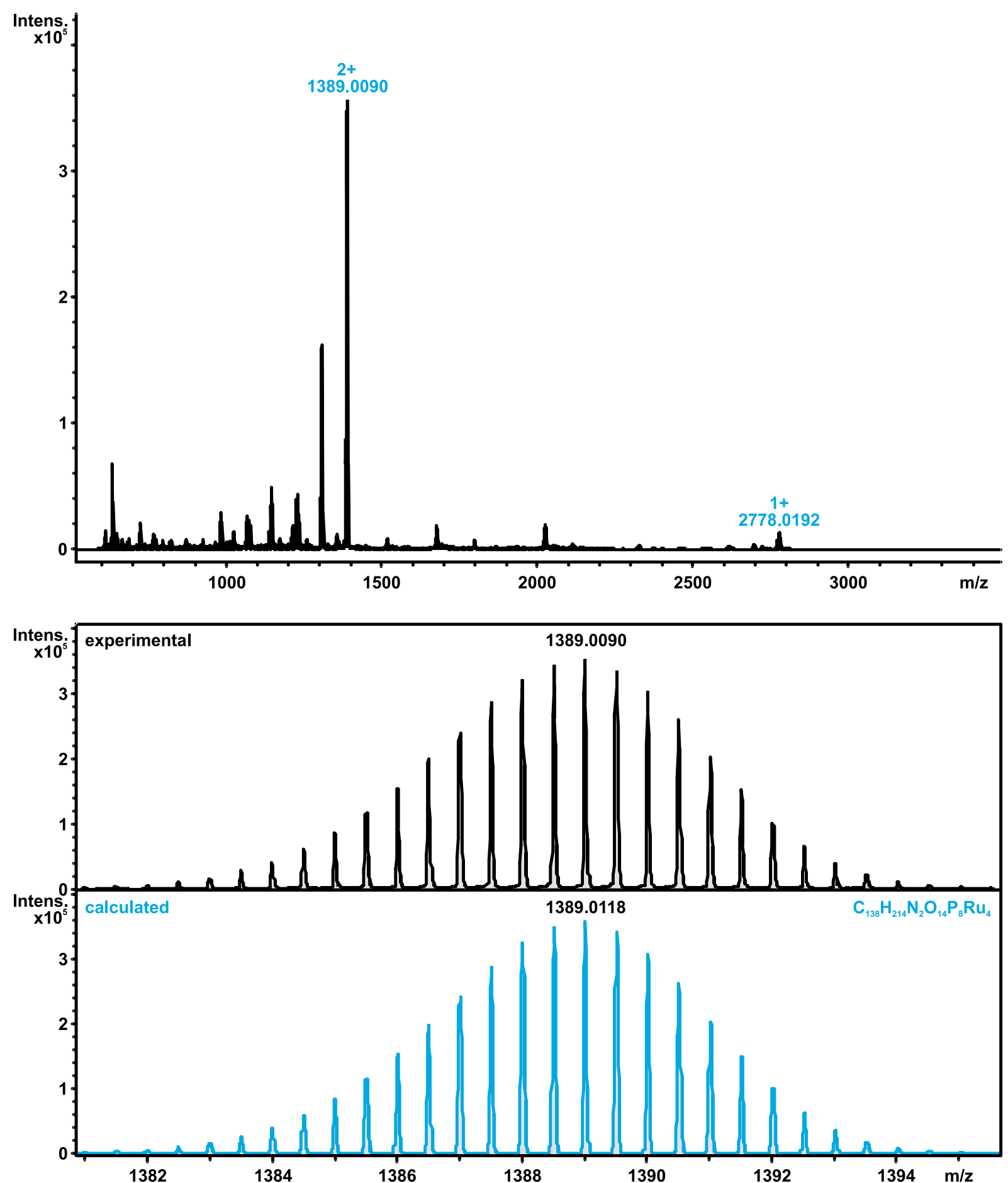

Figure S30. ESI Mass spectrum of macrocycle 2-BN (top), magnification of $[\mathrm{M}]^{2+}$ peak with calculated isotopic pattern (bottom). 

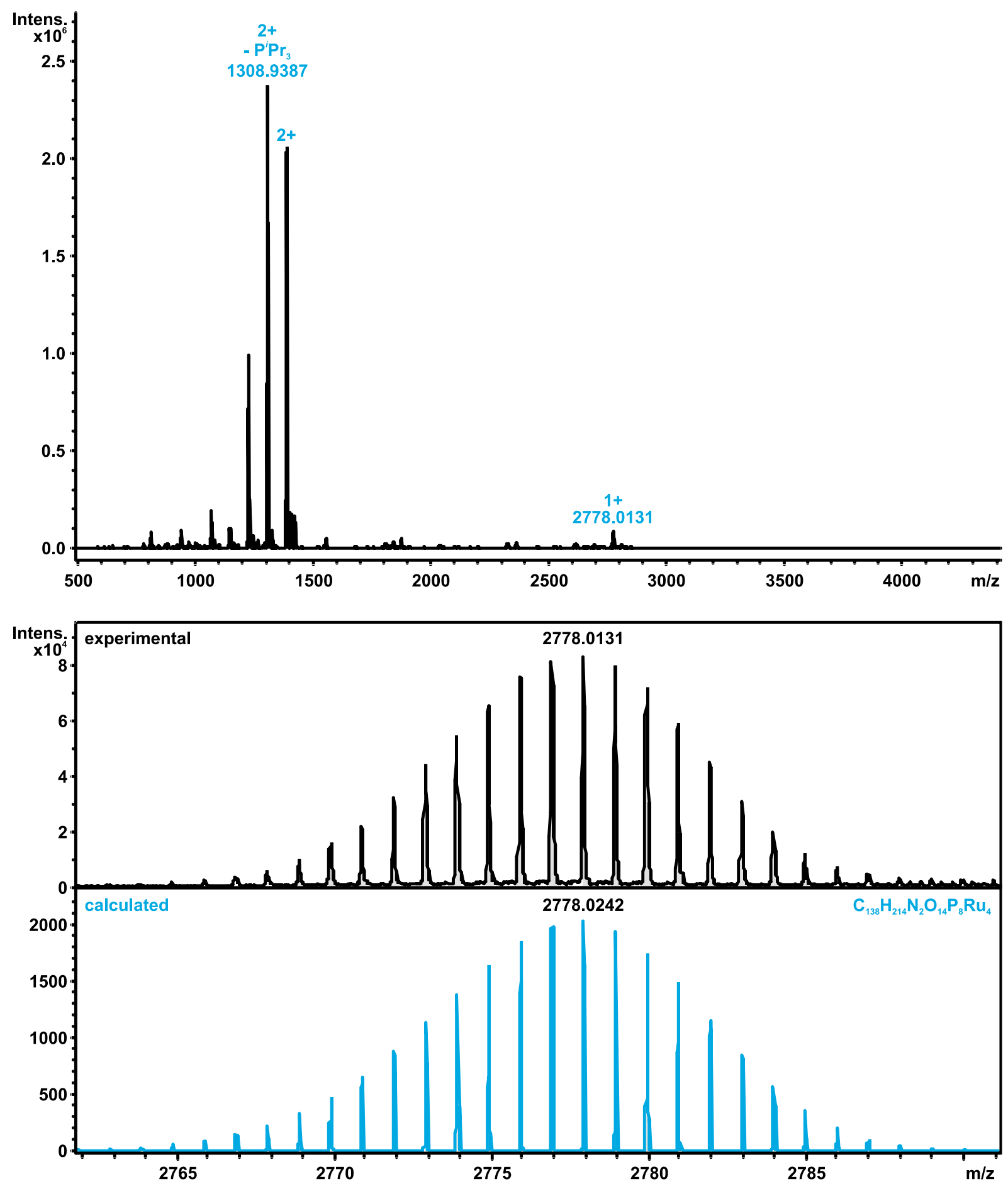

Figure S31. ESI Mass spectrum of macrocycle 2-NB (top), magnification of $[\mathrm{M}]^{2+}$ peak with calculated isotopic pattern (bottom). 


\section{Compound 2-BT:}

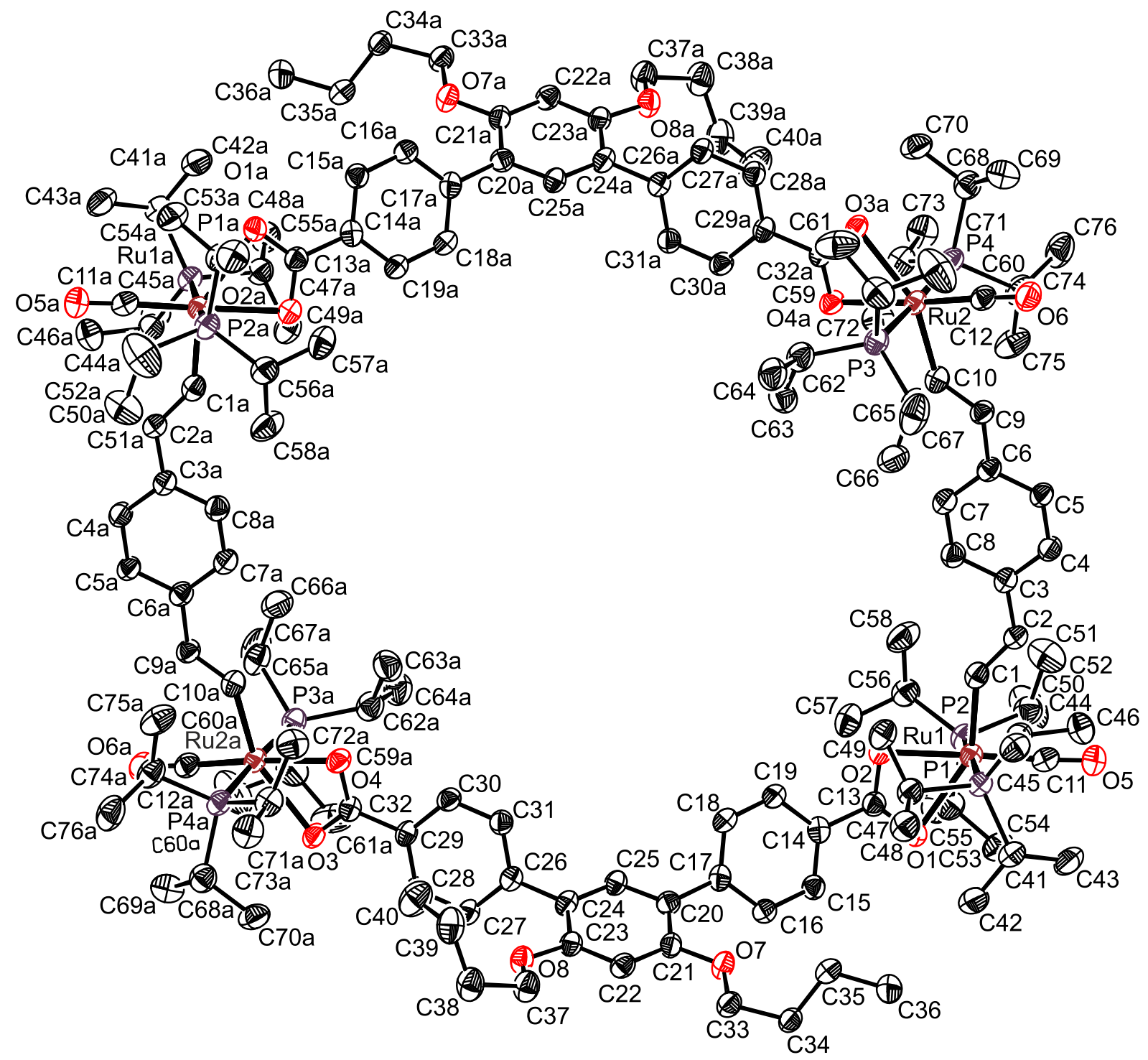

Figure S32. Structure of macrocycle 2-BT as the benzene hexadecasolvate with atom numbering. Benzene solvate molecules as well as hydrogen atoms have been removed for clarity reasons. Ellipsoids are drawn at a $50 \%$ probability level.

Experimental. Single crystals of 2-BT were obtained as yellow blocks by slow diffusion of methanol into a solution of compound 2-BT in benzene. A suitable crystal was selected and mounted on a STOE IPDS-II diffractometer. The crystal was kept at $100.15 \mathrm{~K}$ during data collection. Using Olex2 $2^{(1)}$, the structure was solved with the ShelXT ${ }^{(2)}$ structure solution program using Intrinsic Phasing and refined with the ShelXL ${ }^{(3)}$ refinement package using Least Squares minimisation. 
Table S1. Crystal data and structure refinement for macrocycle $\mathbf{2}-\mathbf{B T} \cdot \mathbf{1 6 C}_{\mathbf{6}} \mathbf{H}_{\mathbf{6}}$.

Empirical formula

Formula weight

Temperature

Wavelength

Crystal system

Space group

Unit cell dimensions

Volume

Z

Density (calculated)

Absorption coefficient

$F(000)$

Crystal size

$2 \Theta$ range for data collection

Index ranges

Reflections collected

Independent reflections

Refinement method

Data / restraints / parameters

Goodness-of-fit on $F^{2}$

Final $R$ indices $[\mathrm{I} \geq 2 \sigma(\mathrm{I})]$

$R$ indices (all data)

Largest diff. peak and hole
$\mathrm{C}_{200} \mathrm{H}_{288} \mathrm{O}_{16} \mathrm{P}_{8} \mathrm{Ru}_{4}$

3600.33

$100.15 \mathrm{~K}$

$0.71073 \AA$

Monoclinic

$P 2_{1} / n$

$a=15.2041(12) \AA$

$\alpha=90^{\circ}$

$b=34.499(3) \AA$

$\beta=107.200(5)^{\circ}$

$c=22.5225(16) \AA$

$\gamma=90^{\circ}$

11285.3(15) $\AA^{3}$

2

$1.060 \mathrm{Mg} / \mathrm{m}^{3}$

$0.369 \mathrm{~mm}^{-1}$

3824.0

$0.4 \times 0.3 \times 0.2 \mathrm{~mm}^{3}$

2.882 to $49.998^{\circ}$

$-17 \leq h \leq 18,-40 \leq k \leq 40,-26 \leq l \leq 26$

88549

$19767\left[R_{\text {int }}=0.0773, R_{\text {sigma }}=0.0633\right]$

Full-matrix least-squares on $\mathrm{F}^{2}$

19767/0/1054

0.952

$R_{1}=0.0515, \mathrm{w} R_{2}=0.1186$

$R_{1}=0.0780, \mathrm{w} R_{2}=0.1289$

0.59 and -0.65 e $\AA^{-3}$ 
Table S2. Selected bond lengths $[\AA]$ for $\mathbf{2}-\mathbf{B T} \cdot \mathbf{1 6} \mathbf{C}_{6} \mathbf{H}_{6}$.

\begin{tabular}{|c|c|c|c|}
\hline $\mathrm{Ru}(1)-\mathrm{P}(1)$ & $2.4313(10)$ & $C(7)-C(8)$ & $1.384(5)$ \\
\hline $\mathrm{Ru}(1)-\mathrm{P}(2)$ & $2.4087(11)$ & $C(9)-C(10)$ & $1.343(5)$ \\
\hline $\mathrm{Ru}(1)-\mathrm{O}(1)$ & $2.344(3)$ & $C(13)-C(14)$ & $1.496(5)$ \\
\hline $\mathrm{Ru}(1)-\mathrm{O}(2)$ & $2.183(2)$ & $C(14)-C(15)$ & $1.385(5)$ \\
\hline $\mathrm{Ru}(1)-\mathrm{C}(1)$ & $2.005(4)$ & $C(14)-C(19)$ & $1.401(5)$ \\
\hline $\operatorname{Ru}(1)-C(11)$ & $1.801(4)$ & $C(15)-C(16)$ & $1.392(5)$ \\
\hline $\operatorname{Ru}(2)-P(3)$ & $2.4244(11)$ & $C(16)-C(17)$ & $1.405(5)$ \\
\hline $\mathrm{Ru}(2)-\mathrm{P}(4)$ & $2.4144(11)$ & $C(17)-C(18)$ & $1.393(5)$ \\
\hline $\mathrm{Ru}(2)-\mathrm{O}(3)$ & $2.304(2)$ & $C(17)-C(20)$ & $1.494(5)$ \\
\hline $\mathrm{Ru}(2)-\mathrm{O}(4)$ & $2.181(3)$ & $C(18)-C(19)$ & $1.389(6)$ \\
\hline $\mathrm{Ru}(2)-\mathrm{C}(10)$ & $2.017(4)$ & $C(20)-C(21)$ & $1.399(5)$ \\
\hline $\mathrm{Ru}(2)-\mathrm{C}(12)$ & $1.797(4)$ & $C(20)-C(25)$ & $1.395(5)$ \\
\hline $\mathrm{O}(1)-\mathrm{C}(13)$ & $1.271(4)$ & $C(21)-C(22)$ & $1.397(5)$ \\
\hline $\mathrm{O}(2)-\mathrm{C}(13)$ & $1.271(4)$ & $C(22)-C(23)$ & $1.397(5)$ \\
\hline $\mathrm{O}(3)-\mathrm{C}(32)$ & $1.258(4)$ & $\mathrm{C}(23)-\mathrm{C}(24)$ & $1.395(5)$ \\
\hline $\mathrm{O}(4)-\mathrm{C}(32)$ & $1.274(4)$ & $C(24)-C(25)$ & $1.392(5)$ \\
\hline $\mathrm{O}(5)-\mathrm{C}(11)$ & $1.174(5)$ & $C(24)-C(26)$ & $1.495(5)$ \\
\hline $\mathrm{O}(6)-\mathrm{C}(12)$ & $1.175(5)$ & $C(26)-C(27)$ & $1.395(5)$ \\
\hline $\mathrm{O}(7)-\mathrm{C}(21)$ & $1.373(4)$ & $C(26)-C(31)$ & $1.388(5)$ \\
\hline $\mathrm{O}(7)-\mathrm{C}(33)$ & $1.435(5)$ & $C(27)-C(28)$ & $1.393(5)$ \\
\hline $\mathrm{O}(8)-\mathrm{C}(23)$ & $1.381(4)$ & $\mathrm{C}(28)-\mathrm{C}(29)$ & $1.387(5)$ \\
\hline $\mathrm{O}(8)-\mathrm{C}(37)$ & $1.439(5)$ & $C(29)-C(30)$ & $1.393(5)$ \\
\hline$C(1)-C(2)$ & $1.338(5)$ & $C(29)-C(32)$ & $1.496(5)$ \\
\hline$C(2)-C(3)$ & $1.473(5)$ & $C(30)-C(31)$ & $1.391(5)$ \\
\hline$C(3)-C(4)$ & $1.399(5)$ & $C(33)-C(34)$ & $1.518(5)$ \\
\hline$C(3)-C(8)$ & $1.399(5)$ & $C(34)-C(35)$ & $1.520(6)$ \\
\hline$C(4)-C(5)$ & $1.385(5)$ & $C(35)-C(36)$ & $1.529(6)$ \\
\hline$C(5)-C(6)$ & $1.402(5)$ & $C(37)-C(38)$ & $1.519(6)$ \\
\hline$C(6)-C(7)$ & $1.414(6)$ & $\mathrm{C}(38)-\mathrm{C}(39)$ & $1.526(7)$ \\
\hline$C(6)-C(9)$ & $1.463(5)$ & $C(39)-C(40)$ & $1.497(7)$ \\
\hline
\end{tabular}


Table S3. Selected bond angles $\left[{ }^{\circ}\right]$ for $\mathbf{2}-\mathbf{B T} \cdot \mathbf{1 6} \mathbf{C}_{6} \mathbf{H}_{6}$.

\begin{tabular}{|c|c|c|c|}
\hline$P(2)-R u(1)-P(1)$ & $173.41(4)$ & $C(10)-C(9)-C(6)$ & $127.1(4)$ \\
\hline $\mathrm{O}(1)-\mathrm{Ru}(1)-\mathrm{P}(1)$ & $86.88(7)$ & $C(9)-C(10)-R u(2)$ & $135.2(3)$ \\
\hline $\mathrm{O}(1)-\mathrm{Ru}(1)-\mathrm{P}(2)$ & $88.14(7)$ & $\mathrm{O}(5)-\mathrm{C}(11)-\mathrm{Ru}(1)$ & $177.1(3)$ \\
\hline $\mathrm{O}(2)-\mathrm{Ru}(1)-\mathrm{P}(1)$ & $91.38(7)$ & $\mathrm{O}(6)-\mathrm{C}(12)-\mathrm{Ru}(2)$ & $177.4(3)$ \\
\hline $\mathrm{O}(2)-\mathrm{Ru}(1)-\mathrm{P}(2)$ & $89.64(7)$ & $\mathrm{O}(1)-\mathrm{C}(13)-\mathrm{O}(2)$ & $119.8(3)$ \\
\hline $\mathrm{O}(2)-\mathrm{Ru}(1)-\mathrm{O}(1)$ & $57.99(9)$ & $\mathrm{O}(1)-\mathrm{C}(13)-\mathrm{C}(14)$ & $120.5(3)$ \\
\hline$C(1)-R u(1)-P(1)$ & $89.88(11)$ & $\mathrm{O}(2)-\mathrm{C}(13)-\mathrm{C}(14)$ & $119.4(3)$ \\
\hline$C(1)-R u(1)-P(2)$ & $96.62(11)$ & $C(15)-C(14)-C(13)$ & $120.4(3)$ \\
\hline $\mathrm{C}(1)-\mathrm{Ru}(1)-\mathrm{O}(1)$ & $148.22(12)$ & $C(15)-C(14)-C(19)$ & 119.2(3) \\
\hline $\mathrm{C}(1)-\mathrm{Ru}(1)-\mathrm{O}(2)$ & $90.53(13)$ & $C(19)-C(14)-C(13)$ & $120.0(3)$ \\
\hline $\mathrm{C}(11)-\mathrm{Ru}(1)-\mathrm{P}(1)$ & $90.69(12)$ & $C(14)-C(15)-C(16)$ & $120.5(3)$ \\
\hline $\mathrm{C}(11)-\mathrm{Ru}(1)-\mathrm{P}(2)$ & $88.08(12)$ & $C(15)-C(16)-C(17)$ & $120.8(4)$ \\
\hline $\mathrm{C}(11)-\mathrm{Ru}(1)-\mathrm{O}(1)$ & $120.19(13)$ & $C(16)-C(17)-C(20)$ & $121.5(3)$ \\
\hline $\mathrm{C}(11)-\mathrm{Ru}(1)-\mathrm{O}(2)$ & $177.14(14)$ & $C(18)-C(17)-C(16)$ & 118.1(3) \\
\hline $\mathrm{C}(11)-\mathrm{Ru}(1)-\mathrm{C}(1)$ & $91.44(16)$ & $C(18)-C(17)-C(20)$ & $120.4(3)$ \\
\hline$P(3)-R u(2)-C(32)$ & $87.95(9)$ & $C(19)-C(18)-C(17)$ & $121.1(3)$ \\
\hline$P(4)-R u(2)-P(3)$ & $177.47(3)$ & $C(18)-C(19)-C(14)$ & $120.3(4)$ \\
\hline$P(4)-R u(2)-C(32)$ & $89.85(8)$ & $C(21)-C(20)-C(17)$ & $121.8(3)$ \\
\hline $\mathrm{O}(3)-\mathrm{Ru}(2)-\mathrm{P}(3)$ & $88.60(7)$ & $C(25)-C(20)-C(17)$ & $120.9(3)$ \\
\hline $\mathrm{O}(3)-\mathrm{Ru}(2)-\mathrm{P}(4)$ & $88.87(7)$ & $C(25)-C(20)-C(21)$ & $117.2(3)$ \\
\hline $\mathrm{O}(3)-\mathrm{Ru}(2)-\mathrm{C}(32)$ & $29.06(10)$ & $\mathrm{O}(7)-\mathrm{C}(21)-\mathrm{C}(20)$ & $116.2(3)$ \\
\hline $\mathrm{O}(4)-\mathrm{Ru}(2)-\mathrm{P}(3)$ & $88.79(7)$ & $\mathrm{O}(7)-\mathrm{C}(21)-\mathrm{C}(22)$ & $122.3(3)$ \\
\hline $\mathrm{O}(4)-\mathrm{Ru}(2)-\mathrm{P}(4)$ & $89.92(7)$ & $C(22)-C(21)-C(20)$ & $121.5(3)$ \\
\hline $\mathrm{O}(4)-\mathrm{Ru}(2)-\mathrm{O}(3)$ & $58.47(9)$ & $C(23)-C(22)-C(21)$ & $119.2(4)$ \\
\hline $\mathrm{O}(4)-\mathrm{Ru}(2)-\mathrm{C}(32)$ & 29.41(10) & $\mathrm{O}(8)-\mathrm{C}(23)-\mathrm{C}(22)$ & $122.5(3)$ \\
\hline $\mathrm{C}(10)-\mathrm{Ru}(2)-\mathrm{P}(3)$ & $90.23(11)$ & $\mathrm{O}(8)-\mathrm{C}(23)-\mathrm{C}(24)$ & $116.6(3)$ \\
\hline $\mathrm{C}(10)-\mathrm{Ru}(2)-\mathrm{P}(4)$ & $92.09(11)$ & $C(24)-C(23)-C(22)$ & $120.9(3)$ \\
\hline $\mathrm{C}(10)-\mathrm{Ru}(2)-\mathrm{O}(3)$ & $155.37(13)$ & $C(23)-C(24)-C(26)$ & $120.4(3)$ \\
\hline $\mathrm{C}(10)-\mathrm{Ru}(2)-\mathrm{O}(4)$ & $96.92(13)$ & $C(25)-C(24)-C(23)$ & 118.1(3) \\
\hline$C(10)-R u(2)-C(32)$ & $126.31(14)$ & $C(25)-C(24)-C(26)$ & $121.3(3)$ \\
\hline
\end{tabular}




\begin{tabular}{|c|c|c|c|}
\hline $\mathrm{C}(12)-\mathrm{Ru}(2)-\mathrm{P}(3)$ & $91.55(13)$ & $C(24)-C(25)-C(20)$ & $123.1(4)$ \\
\hline$C(12)-R u(2)-P(4)$ & $89.58(13)$ & $C(27)-C(26)-C(24)$ & 121.2(3) \\
\hline $\mathrm{C}(12)-\mathrm{Ru}(2)-\mathrm{O}(3)$ & $117.49(13)$ & $C(31)-C(26)-C(24)$ & $120.1(3)$ \\
\hline $\mathrm{C}(12)-\mathrm{Ru}(2)-\mathrm{O}(4)$ & 175.94(13) & $C(31)-C(26)-C(27)$ & $118.8(3)$ \\
\hline$C(12)-R u(2)-C(10)$ & $87.13(16)$ & $C(28)-C(27)-C(26)$ & $120.4(3)$ \\
\hline $\mathrm{C}(12)-\mathrm{Ru}(2)-\mathrm{C}(32)$ & $146.55(14)$ & $C(29)-C(28)-C(27)$ & $120.0(4)$ \\
\hline $\mathrm{C}(13)-\mathrm{O}(1)-\mathrm{Ru}(1)$ & $87.2(2)$ & $C(28)-C(29)-C(30)$ & $120.1(3)$ \\
\hline $\mathrm{C}(13)-\mathrm{O}(2)-\mathrm{Ru}(1)$ & $94.5(2)$ & $C(28)-C(29)-C(32)$ & $120.5(3)$ \\
\hline $\mathrm{C}(32)-\mathrm{O}(3)-\mathrm{Ru}(2)$ & $88.1(2)$ & $C(30)-C(29)-C(32)$ & 119.4(3) \\
\hline $\mathrm{C}(32)-\mathrm{O}(4)-\mathrm{Ru}(2)$ & $93.3(2)$ & $C(31)-C(30)-C(29)$ & $119.3(3)$ \\
\hline$C(21)-O(7)-C(33)$ & 119.2(3) & $C(26)-C(31)-C(30)$ & $121.3(4)$ \\
\hline $\mathrm{C}(23)-\mathrm{O}(8)-\mathrm{C}(37)$ & 117.0(3) & $\mathrm{O}(3)-\mathrm{C}(32)-\mathrm{Ru}(2)$ & $62.80(18)$ \\
\hline$C(2)-C(1)-R u(1)$ & 139.7(3) & $\mathrm{O}(3)-\mathrm{C}(32)-\mathrm{O}(4)$ & $120.0(3)$ \\
\hline$C(1)-C(2)-C(3)$ & $126.5(3)$ & $\mathrm{O}(3)-\mathrm{C}(32)-\mathrm{C}(29)$ & $120.6(3)$ \\
\hline$C(4)-C(3)-C(2)$ & $120.2(3)$ & $\mathrm{O}(4)-\mathrm{C}(32)-\mathrm{Ru}(2)$ & $57.24(17)$ \\
\hline$C(4)-C(3)-C(8)$ & $116.8(3)$ & $\mathrm{O}(4)-\mathrm{C}(32)-\mathrm{C}(29)$ & $119.4(3)$ \\
\hline$C(8)-C(3)-C(2)$ & $123.0(4)$ & $C(29)-C(32)-R u(2)$ & $176.4(3)$ \\
\hline$C(5)-C(4)-C(3)$ & $121.4(3)$ & $\mathrm{O}(7)-\mathrm{C}(33)-\mathrm{C}(34)$ & $106.7(3)$ \\
\hline$C(4)-C(5)-C(6)$ & $122.4(4)$ & $C(33)-C(34)-C(35)$ & 113.3(3) \\
\hline$C(5)-C(6)-C(7)$ & $115.8(3)$ & $C(34)-C(35)-C(36)$ & $112.1(3)$ \\
\hline$C(5)-C(6)-C(9)$ & $121.0(4)$ & $\mathrm{O}(8)-\mathrm{C}(37)-\mathrm{C}(38)$ & 109.3(3) \\
\hline$C(7)-C(6)-C(9)$ & 123.2(3) & $C(37)-C(38)-C(39)$ & 114.1(4) \\
\hline$C(8)-C(7)-C(6)$ & $121.6(3)$ & $C(40)-C(39)-C(38)$ & $114.5(4)$ \\
\hline$C(7)-C(8)-C(3)$ & $121.9(4)$ & & \\
\hline
\end{tabular}




\section{Compound 2-NB:}

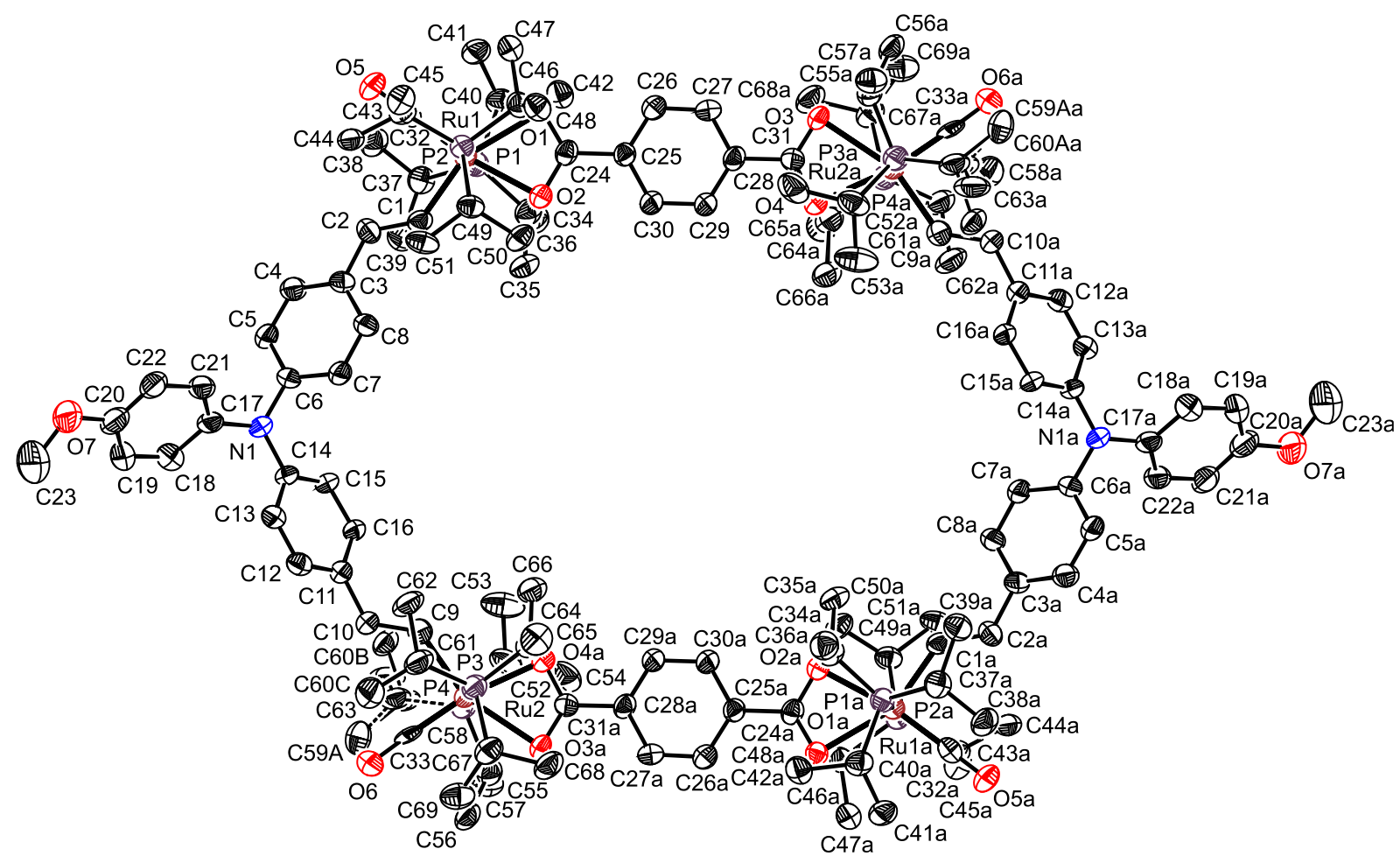

Figure S33. Structure of macrocycle 2-NB as the benzene hexasolvate with atom numbering. Benzene solvate molecules as well as hydrogen atoms have been removed for clarity reasons. Ellipsoids are drawn at a $50 \%$ probability level.

Experimental. Single crystals of 2-NB were obtained as yellow blocks by slow diffusion of methanol into a solution of compound 2-NB in benzene. A suitable crystal was selected and mounted on a STOE IPDS-II diffractometer. The crystal was kept at $100.15 \mathrm{~K}$ during data collection. Using Olex $2^{(1)}$, the structure was solved with the ShelXT ${ }^{(2)}$ structure solution program using Intrinsic Phasing and refined with the ShelXL ${ }^{(3)}$ refinement package using Least Squares minimisation. 
Table S4. Crystal data and structure refinement for macrocycle $\mathbf{2}-\mathbf{N B} \cdot \mathbf{6} \mathbf{C}_{6} \mathbf{H}_{6}$.

Empirical formula

Formula weight

Temperature

Wavelength

Crystal system

Space group

Unit cell dimensions

Volume

Z

Density (calculated)

Absorption coefficient

$F(000)$

Crystal size

$2 \Theta$ range for data collection

Index ranges

Reflections collected

Independent reflections

Refinement method

Data / restraints / parameters

Goodness-of-fit on $F^{2}$

Final $R$ indices $[\mathrm{I} \geq 2 \sigma(\mathrm{I})]$

$R$ indices (all data)

Largest diff. peak and hole
$\mathrm{C}_{138} \mathrm{H}_{214} \mathrm{~N}_{2} \mathrm{O}_{14} \mathrm{P}_{8} \mathrm{Ru}_{4}$

2777.14

$100.15 \mathrm{~K}$

$0.71073 \AA$

Triclinic

$P-1$

$a=11.0870(9) \AA$

$\alpha=98.853(8)^{\circ}$

$b=19.9232(17) \AA$

$\beta=100.783(8)^{\circ}$

$c=21.192(2) \AA$

$\gamma=104.906(6)^{\circ}$

4342.0(7) $\AA^{3}$

1

$1.062 \mathrm{Mg} / \mathrm{m}^{3}$

$0.462 \mathrm{~mm}^{-1}$

1464.0

$0.1 \times 0.2 \times 0.3 \mathrm{~mm}^{3}$

3.874 to $51.996^{\circ}$

$-13 \leq h \leq 13,-24 \leq k \leq 24,-26 \leq l \leq 26$

54977

$17058\left[R_{\text {int }}=0.1729, R_{\text {sigma }}=0.1343\right]$

Full-matrix least-squares on $\mathrm{F}^{2}$

$17058 / 3 / 787$

0.973

$R_{1}=0.1051, \mathrm{w} R_{2}=0.2561$

$R_{1}=0.1431, \mathrm{w} R_{2}=0.2822$

2.42 and $-1.84{\mathrm{e} \AA^{-3}}^{-3}$ 
Table S5. Selected bond lengths $[\AA]$ for $\mathbf{2}-\mathbf{N B} \cdot \mathbf{6} \mathbf{C}_{6} \mathbf{H}_{6}$.

\begin{tabular}{|c|c|c|c|}
\hline $\mathrm{Ru}(1)-\mathrm{P}(1)$ & $2.410(2)$ & $C(4)-C(5)$ & $1.388(11)$ \\
\hline $\mathrm{Ru}(1)-\mathrm{P}(2)$ & $2.428(2)$ & $C(5)-C(6)$ & $1.376(11)$ \\
\hline $\mathrm{Ru}(1)-\mathrm{O}(1)$ & $2.271(5)$ & $C(6)-C(7)$ & $1.412(11)$ \\
\hline $\mathrm{Ru}(1)-\mathrm{O}(2)$ & $2.191(5)$ & $C(7)-C(8)$ & $1.401(11)$ \\
\hline $\mathrm{Ru}(1)-\mathrm{C}(1)$ & $2.022(7)$ & $C(9)-C(10)$ & $1.360(11)$ \\
\hline $\mathrm{Ru}(1)-\mathrm{C}(32)$ & $1.797(8)$ & $C(10)-C(11)$ & $1.458(10)$ \\
\hline $\mathrm{Ru}(2)-\mathrm{P}(3)$ & $2.401(3)$ & $\mathrm{C}(11)-\mathrm{C}(12)$ & $1.403(11)$ \\
\hline $\operatorname{Ru}(2)-P(4)$ & $2.435(2)$ & $C(11)-C(16)$ & $1.394(11)$ \\
\hline $\mathrm{Ru}(2)-\mathrm{O}(3)$ & $2.309(5)$ & $C(12)-C(13)$ & $1.368(11)$ \\
\hline $\mathrm{Ru}(2)-\mathrm{O}(4)$ & $2.189(5)$ & $C(13)-C(14)$ & $1.384(11)$ \\
\hline $\mathrm{Ru}(2)-\mathrm{C}(9)$ & $2.021(8)$ & $C(14)-C(15)$ & $1.396(11)$ \\
\hline $\mathrm{Ru}(2)-\mathrm{C}(33)$ & $1.760(8)$ & $C(15)-C(16)$ & $1.393(10)$ \\
\hline $\mathrm{O}(1)-\mathrm{C}(24)$ & $1.240(9)$ & $C(17)-C(18)$ & $1.387(12)$ \\
\hline $\mathrm{O}(2)-\mathrm{C}(24)$ & $1.298(9)$ & $C(17)-C(22)$ & $1.387(12)$ \\
\hline $\mathrm{O}(3)-\mathrm{C}(31)$ & $1.255(9)$ & $C(18)-C(19)$ & $1.393(13)$ \\
\hline $\mathrm{O}(4)-\mathrm{C}(31)$ & $1.274(9)$ & $C(19)-C(20)$ & $1.340(15)$ \\
\hline $\mathrm{O}(5)-\mathrm{C}(32)$ & $1.186(10)$ & $C(20)-C(21)$ & $1.391(15)$ \\
\hline $\mathrm{O}(6)-\mathrm{C}(33)$ & $1.223(10)$ & $C(21)-C(22)$ & $1.402(12)$ \\
\hline $\mathrm{O}(7)-\mathrm{C}(20)$ & $1.405(12)$ & $C(24)-C(25)$ & $1.483(10)$ \\
\hline $\mathrm{O}(7)-\mathrm{C}(23)$ & $1.451(19)$ & $C(25)-C(26)$ & $1.407(11)$ \\
\hline$N(1)-C(6)$ & $1.443(10)$ & $\mathrm{C}(25)-\mathrm{C}(30)$ & $1.413(10)$ \\
\hline $\mathrm{N}(1)-\mathrm{C}(14)$ & $1.412(9)$ & $C(26)-C(27)$ & $1.383(11)$ \\
\hline $\mathrm{N}(1)-\mathrm{C}(17)$ & $1.441(11)$ & $C(27)-C(28)$ & $1.400(10)$ \\
\hline$C(1)-C(2)$ & $1.341(11)$ & $\mathrm{C}(28)-\mathrm{C}(29)$ & $1.385(11)$ \\
\hline$C(2)-C(3)$ & $1.469(10)$ & $\mathrm{C}(28)-\mathrm{C}(31)$ & $1.485(10)$ \\
\hline$C(3)-C(4)$ & $1.369(11)$ & $C(29)-C(30)$ & $1.379(10)$ \\
\hline$C(3)-C(8)$ & $1.399(11)$ & & \\
\hline
\end{tabular}


Table S6. Selected bond angles $\left[{ }^{\circ}\right]$ for $\mathbf{2}-\mathbf{N B} \cdot \mathbf{6 C}_{6} \mathbf{H}_{6}$.

\begin{tabular}{|c|c|c|c|}
\hline $\mathrm{P}(1)-\mathrm{Ru}(1)-\mathrm{P}(2)$ & $175.32(7)$ & $C(8)-C(3)-C(2)$ & $121.4(7)$ \\
\hline $\mathrm{P}(1)-\mathrm{Ru}(1)-\mathrm{C}(24)$ & $85.74(18)$ & $C(3)-C(4)-C(5)$ & $122.4(8)$ \\
\hline $\mathrm{P}(2)-\mathrm{Ru}(1)-\mathrm{C}(24)$ & 89.61(18) & $C(6)-C(5)-C(4)$ & $119.9(7)$ \\
\hline $\mathrm{O}(1)-\mathrm{Ru}(1)-\mathrm{P}(1)$ & $84.69(14)$ & $C(5)-C(6)-N(1)$ & $121.2(7)$ \\
\hline $\mathrm{O}(1)-\mathrm{Ru}(1)-\mathrm{P}(2)$ & $90.95(14)$ & $C(5)-C(6)-C(7)$ & $119.6(7)$ \\
\hline $\mathrm{O}(1)-\mathrm{Ru}(1)-\mathrm{C}(24)$ & $28.7(2)$ & $C(7)-C(6)-N(1)$ & $119.1(7)$ \\
\hline $\mathrm{O}(2)-\mathrm{Ru}(1)-\mathrm{P}(1)$ & $88.22(15)$ & $C(8)-C(7)-C(6)$ & $118.9(7)$ \\
\hline $\mathrm{O}(2)-\mathrm{Ru}(1)-\mathrm{P}(2)$ & $88.06(15)$ & $C(3)-C(8)-C(7)$ & $121.1(7)$ \\
\hline $\mathrm{O}(2)-\mathrm{Ru}(1)-\mathrm{O}(1)$ & $58.96(18)$ & $C(10)-C(9)-R u(2)$ & $133.5(6)$ \\
\hline $\mathrm{O}(2)-\mathrm{Ru}(1)-\mathrm{C}(24)$ & $30.2(2)$ & $C(9)-C(10)-C(11)$ & $126.4(7)$ \\
\hline$C(1)-R u(1)-P(1)$ & $91.7(2)$ & $C(12)-C(11)-C(10)$ & $118.6(7)$ \\
\hline$C(1)-R u(1)-P(2)$ & $91.6(2)$ & $C(16)-C(11)-C(10)$ & $124.7(7)$ \\
\hline $\mathrm{C}(1)-\mathrm{Ru}(1)-\mathrm{O}(1)$ & $155.3(2)$ & $C(16)-C(11)-C(12)$ & 116.7(7) \\
\hline $\mathrm{C}(1)-\mathrm{Ru}(1)-\mathrm{O}(2)$ & $96.6(2)$ & $C(13)-C(12)-C(11)$ & $122.2(7)$ \\
\hline$C(1)-R u(1)-C(24)$ & $126.7(3)$ & $C(12)-C(13)-C(14)$ & $120.9(8)$ \\
\hline $\mathrm{C}(32)-\mathrm{Ru}(1)-\mathrm{P}(1)$ & $91.8(3)$ & $C(13)-C(14)-N(1)$ & $122.4(7)$ \\
\hline $\mathrm{C}(32)-\mathrm{Ru}(1)-\mathrm{P}(2)$ & $91.4(3)$ & $C(13)-C(14)-C(15)$ & $118.3(7)$ \\
\hline $\mathrm{C}(32)-\mathrm{Ru}(1)-\mathrm{O}(1)$ & $112.6(3)$ & $C(15)-C(14)-N(1)$ & 119.3(7) \\
\hline $\mathrm{C}(32)-\mathrm{Ru}(1)-\mathrm{O}(2)$ & $171.5(3)$ & $C(16)-C(15)-C(14)$ & $120.4(7)$ \\
\hline $\mathrm{C}(32)-\mathrm{Ru}(1)-\mathrm{C}(1)$ & $91.9(3)$ & $C(15)-C(16)-C(11)$ & $121.4(7)$ \\
\hline $\mathrm{C}(32)-\mathrm{Ru}(1)-\mathrm{C}(24)$ & 141.3(3) & $C(18)-C(17)-N(1)$ & $120.6(8)$ \\
\hline$P(3)-R u(2)-P(4)$ & $175.65(8)$ & $C(22)-C(17)-N(1)$ & $121.8(7)$ \\
\hline$P(3)-R u(2)-C(31)$ & $89.6(2)$ & $C(22)-C(17)-C(18)$ & 117.3(8) \\
\hline$P(4)-R u(2)-C(31)$ & $86.1(2)$ & $C(17)-C(18)-C(19)$ & $120.8(9)$ \\
\hline $\mathrm{O}(3)-\mathrm{Ru}(2)-\mathrm{P}(3)$ & $89.19(16)$ & $C(20)-C(19)-C(18)$ & $120.8(9)$ \\
\hline $\mathrm{O}(3)-\mathrm{Ru}(2)-\mathrm{P}(4)$ & $86.98(15)$ & $C(19)-C(20)-O(7)$ & $123.6(10)$ \\
\hline $\mathrm{O}(3)-\mathrm{Ru}(2)-\mathrm{C}(31)$ & $29.0(2)$ & $C(19)-C(20)-C(21)$ & $120.9(9)$ \\
\hline $\mathrm{O}(4)-\mathrm{Ru}(2)-\mathrm{P}(3)$ & $89.34(16)$ & $\mathrm{C}(21)-\mathrm{C}(20)-\mathrm{O}(7)$ & $115.5(9)$ \\
\hline $\mathrm{O}(4)-\mathrm{Ru}(2)-\mathrm{P}(4)$ & $86.90(16)$ & $C(20)-C(21)-C(22)$ & 117.9(9) \\
\hline
\end{tabular}




\begin{tabular}{|c|c|c|c|}
\hline $\mathrm{O}(4)-\mathrm{Ru}(2)-\mathrm{O}(3)$ & $58.46(19)$ & $C(17)-C(22)-C(21)$ & $122.1(8)$ \\
\hline $\mathrm{O}(4)-\mathrm{Ru}(2)-\mathrm{C}(31)$ & $29.5(2)$ & $\mathrm{O}(1)-\mathrm{C}(24)-\mathrm{Ru}(1)$ & $61.7(4)$ \\
\hline $\mathrm{C}(9)-\mathrm{Ru}(2)-\mathrm{P}(3)$ & 91.1(3) & $\mathrm{O}(1)-\mathrm{C}(24)-\mathrm{O}(2)$ & $119.8(6)$ \\
\hline$C(9)-R u(2)-P(4)$ & $91.7(3)$ & $\mathrm{O}(1)-\mathrm{C}(24)-\mathrm{C}(25)$ & $121.7(7)$ \\
\hline $\mathrm{C}(9)-\mathrm{Ru}(2)-\mathrm{O}(3)$ & $158.6(3)$ & $\mathrm{O}(2)-\mathrm{C}(24)-\mathrm{Ru}(1)$ & $58.1(3)$ \\
\hline $\mathrm{C}(9)-\mathrm{Ru}(2)-\mathrm{O}(4)$ & $100.1(3)$ & $\mathrm{O}(2)-\mathrm{C}(24)-\mathrm{C}(25)$ & $118.5(6)$ \\
\hline$C(9)-R u(2)-C(31)$ & 129.6(3) & $C(25)-C(24)-R u(1)$ & $176.4(6)$ \\
\hline $\mathrm{C}(33)-\mathrm{Ru}(2)-\mathrm{P}(3)$ & $91.8(3)$ & $C(26)-C(25)-C(24)$ & $120.2(7)$ \\
\hline $\mathrm{C}(33)-\mathrm{Ru}(2)-\mathrm{P}(4)$ & $91.6(3)$ & $C(26)-C(25)-C(30)$ & 119.2(7) \\
\hline $\mathrm{C}(33)-\mathrm{Ru}(2)-\mathrm{O}(3)$ & $111.7(2)$ & $C(30)-C(25)-C(24)$ & $120.6(7)$ \\
\hline $\mathrm{C}(33)-\mathrm{Ru}(2)-\mathrm{O}(4)$ & $170.1(2)$ & $C(27)-C(26)-C(25)$ & $119.3(7)$ \\
\hline $\mathrm{C}(33)-\mathrm{Ru}(2)-\mathrm{C}(9)$ & $89.7(3)$ & $C(26)-C(27)-C(28)$ & $121.0(7)$ \\
\hline$C(33)-R u(2)-C(31)$ & $140.7(3)$ & $C(27)-C(28)-C(31)$ & $119.5(7)$ \\
\hline $\mathrm{C}(24)-\mathrm{O}(1)-\mathrm{Ru}(1)$ & $89.5(4)$ & $C(29)-C(28)-C(27)$ & $119.8(7)$ \\
\hline $\mathrm{C}(24)-\mathrm{O}(2)-\mathrm{Ru}(1)$ & $91.7(4)$ & $C(29)-C(28)-C(31)$ & $120.6(7)$ \\
\hline $\mathrm{C}(31)-\mathrm{O}(3)-\mathrm{Ru}(2)$ & $87.8(4)$ & $C(30)-C(29)-C(28)$ & $120.1(7)$ \\
\hline $\mathrm{C}(31)-\mathrm{O}(4)-\mathrm{Ru}(2)$ & $92.8(4)$ & $C(29)-C(30)-C(25)$ & $120.6(7)$ \\
\hline$C(20)-O(7)-C(23)$ & $114.8(11)$ & $\mathrm{O}(3)-\mathrm{C}(31)-\mathrm{Ru}(2)$ & 63.2(4) \\
\hline$C(14)-N(1)-C(6)$ & $121.3(6)$ & $\mathrm{O}(3)-\mathrm{C}(31)-\mathrm{O}(4)$ & $120.8(7)$ \\
\hline$C(14)-N(1)-C(17)$ & $120.1(6)$ & $\mathrm{O}(3)-\mathrm{C}(31)-\mathrm{C}(28)$ & $120.3(7)$ \\
\hline$C(17)-N(1)-C(6)$ & $115.6(6)$ & $\mathrm{O}(4)-\mathrm{C}(31)-\mathrm{Ru}(2)$ & $57.7(4)$ \\
\hline$C(2)-C(1)-R u(1)$ & $134.9(6)$ & $\mathrm{O}(4)-\mathrm{C}(31)-\mathrm{C}(28)$ & $118.8(7)$ \\
\hline$C(1)-C(2)-C(3)$ & $127.2(8)$ & $C(28)-C(31)-R u(2)$ & $175.8(6)$ \\
\hline$C(4)-C(3)-C(2)$ & $120.6(7)$ & $\mathrm{O}(5)-\mathrm{C}(32)-\mathrm{Ru}(1)$ & $178.8(7)$ \\
\hline $\mathrm{C}(4)-\mathrm{C}(3)-\mathrm{C}(8)$ & $118.0(7)$ & $\mathrm{O}(6)-\mathrm{C}(33)-\mathrm{Ru}(2)$ & $177.6(6)$ \\
\hline
\end{tabular}




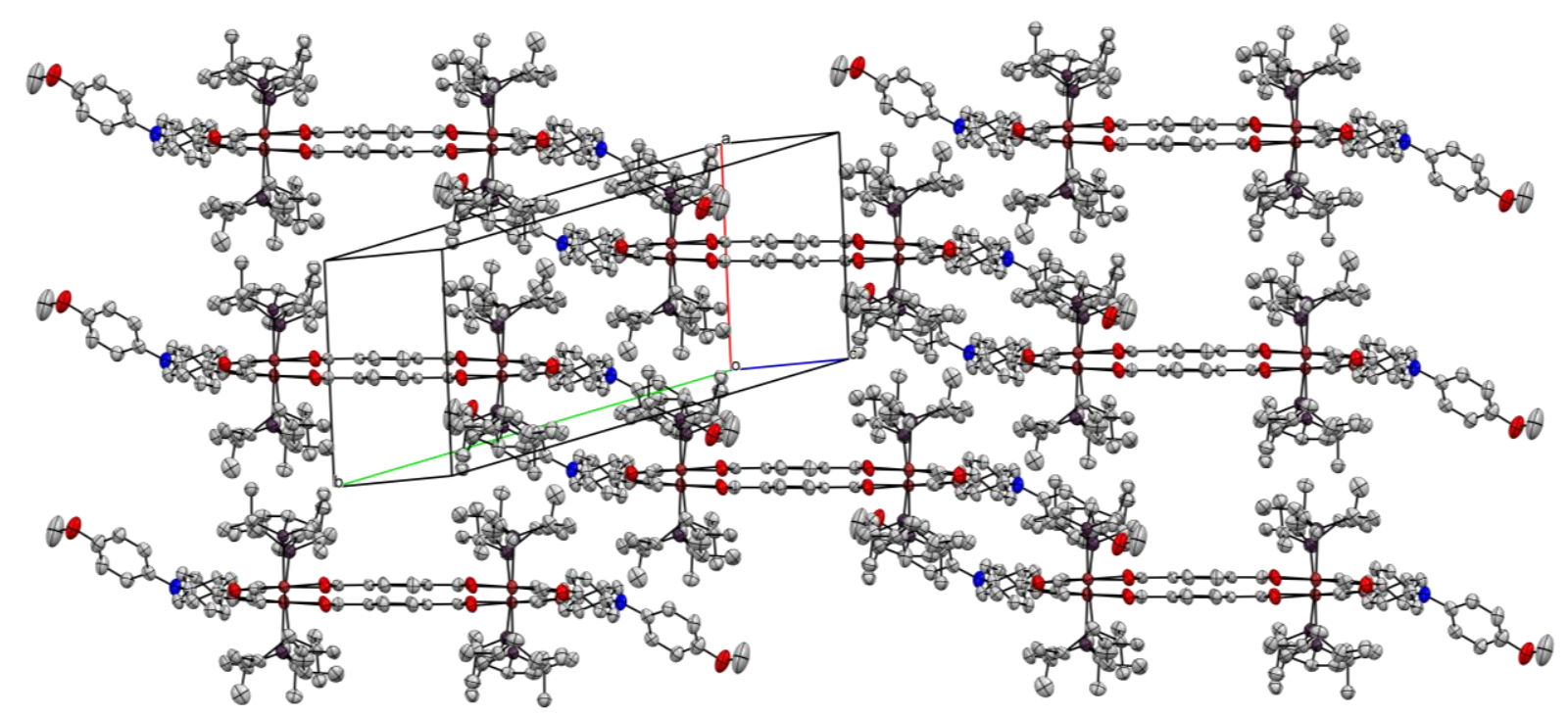

Figure S34. Packing diagram of individual 2-NB molecules in the crystal. Benzene solvate molecules as well as hydrogen atoms have been removed for clarity reasons. Ellipsoids are drawn at a $50 \%$ probability level.

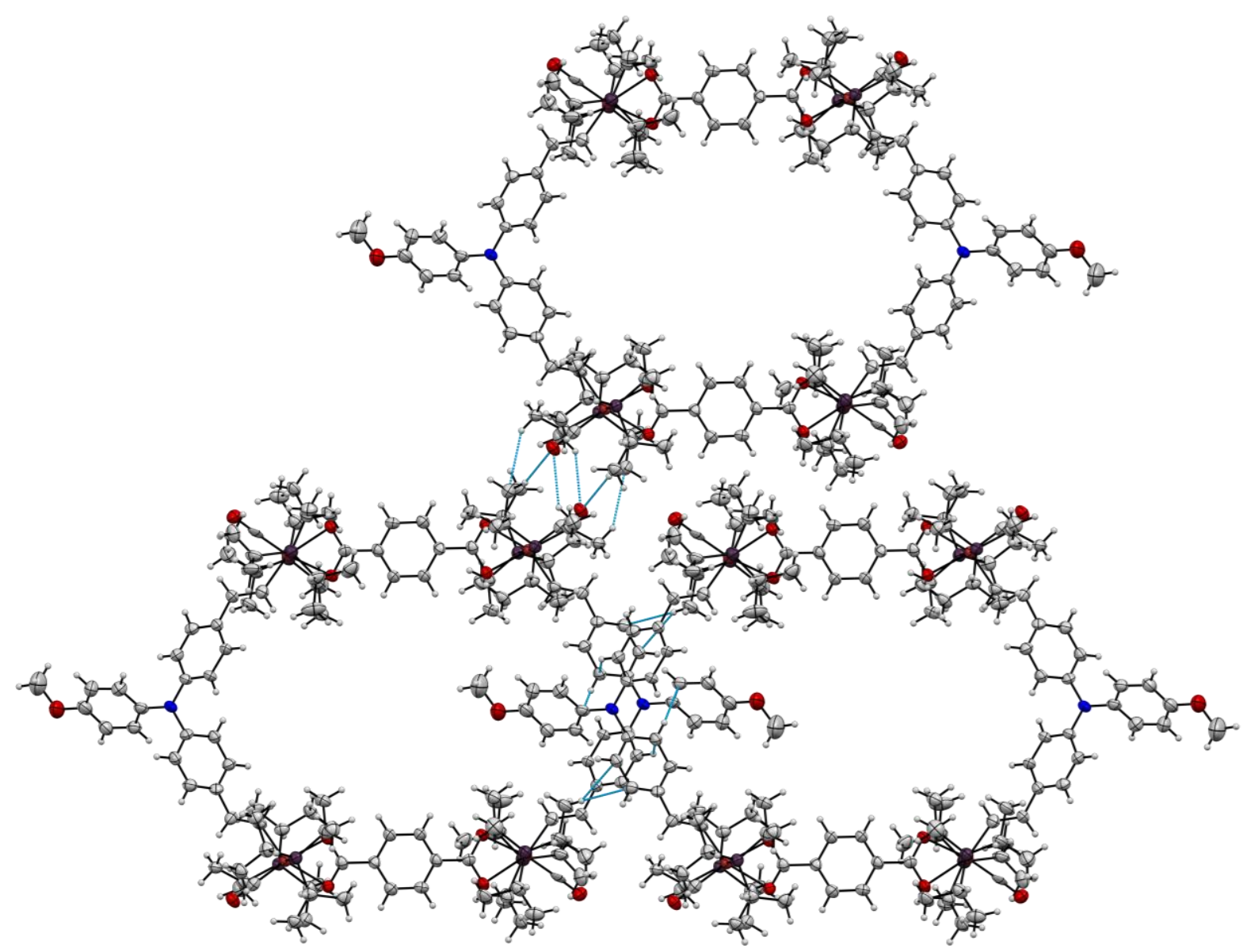

Figure S35. Hydrogen bonding motifs in the molecular structure of metallacycle 2-NB. Benzene solvate molecules have been removed for clarity reasons. Ellipsoids are drawn at a 50\% probability level. 


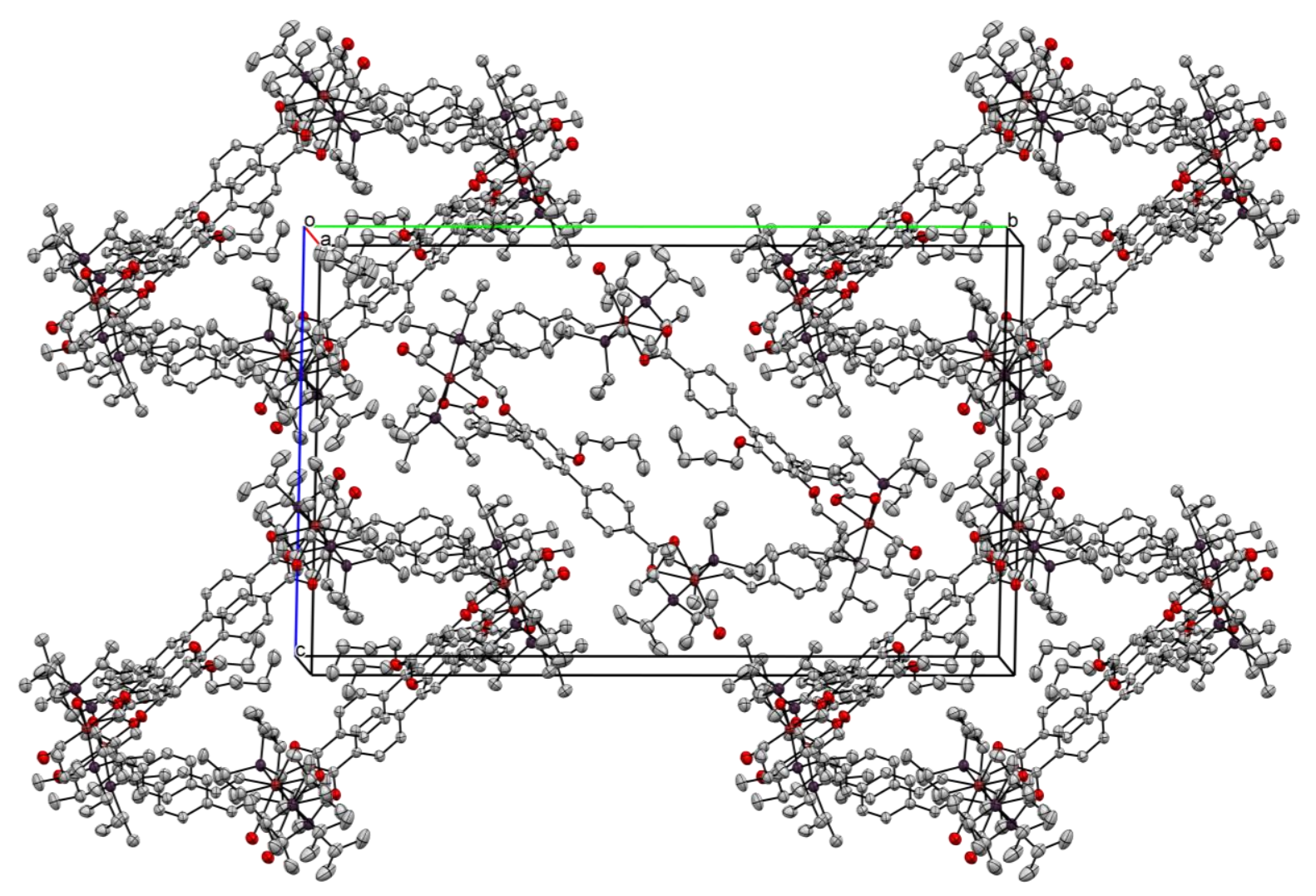

Figure S36. Packing diagram of individual 2-BT molecules in the crystal. Benzene solvate molecules as well as hydrogen atoms have been removed for clarity reasons. Ellipsoids are drawn at a 50\% probability level.

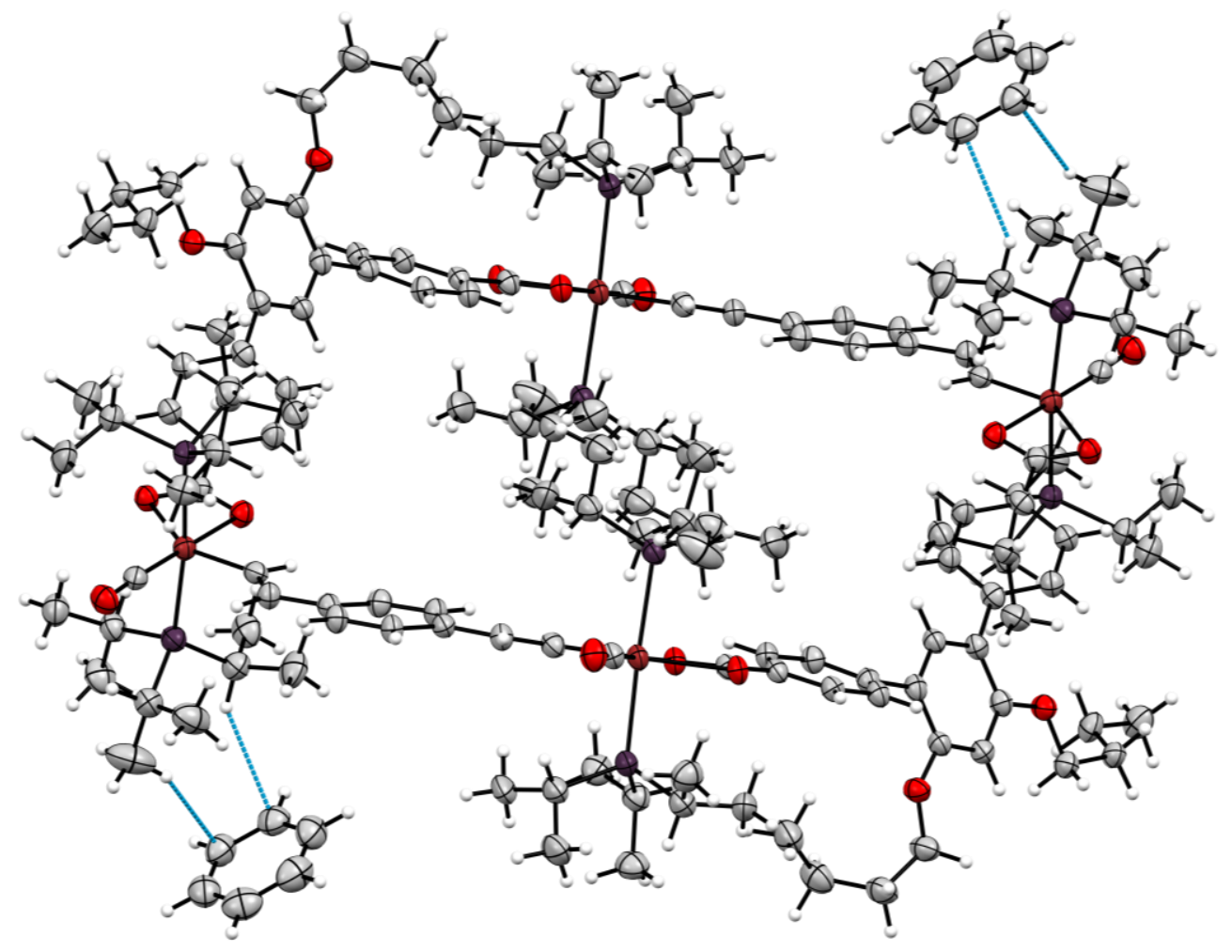

Figure S37. Hydrogen bonding motif in the molecular structure of metallacycle 2-BT. Benzene solvate molecules have been removed for clarity reasons. Ellipsoids are drawn at a 50\% probability level. 

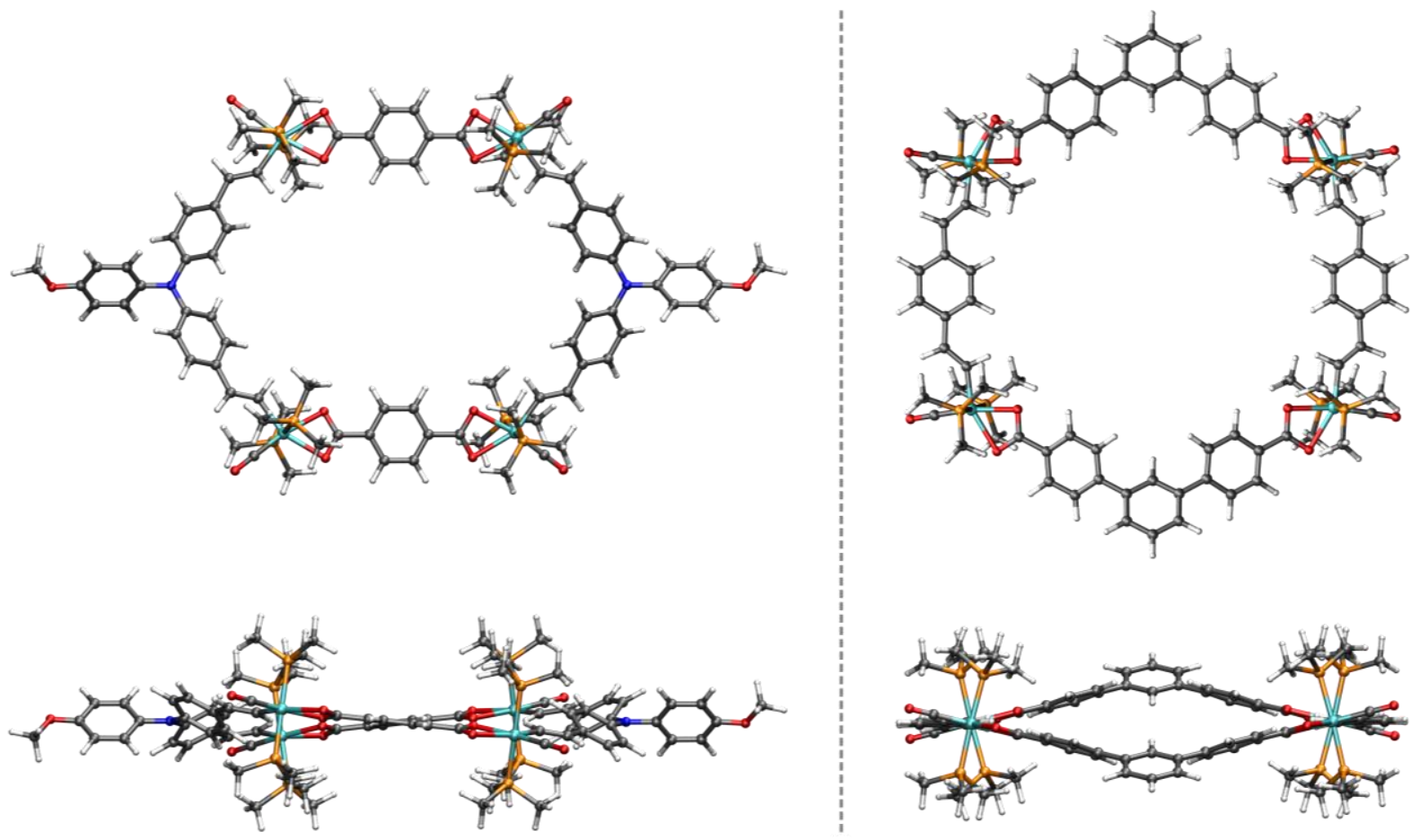

Figure S38. DFT-optimized structures of model compounds 2-NB ${ }^{\mathrm{Me}}$ (left) and 2-BT ${ }^{\mathrm{Me}}$ (right). 


\section{Cyclic Voltammetry}

\section{Compound 2-BT}
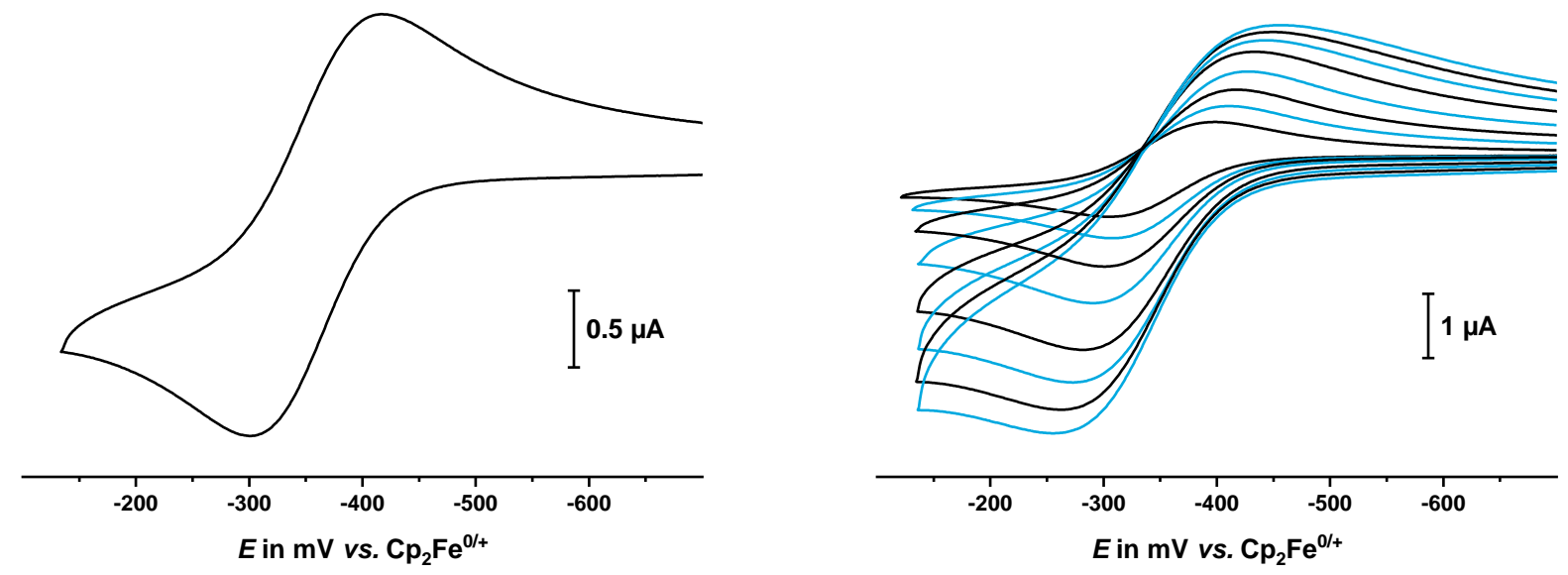

Figure S39. Cyclic voltammograms for the first composite wave of macrocycle 2-BT at $v=100 \mathrm{mV} / \mathrm{s}$ (left) and at $v=25,50,100,200,400,600,800,1000 \mathrm{mV} / \mathrm{s}$ (right) in $\mathrm{CH}_{2} \mathrm{Cl}_{2} /{ }^{n} \mathrm{Bu}_{4} \mathrm{NBAr}^{\mathrm{F} 24}$.
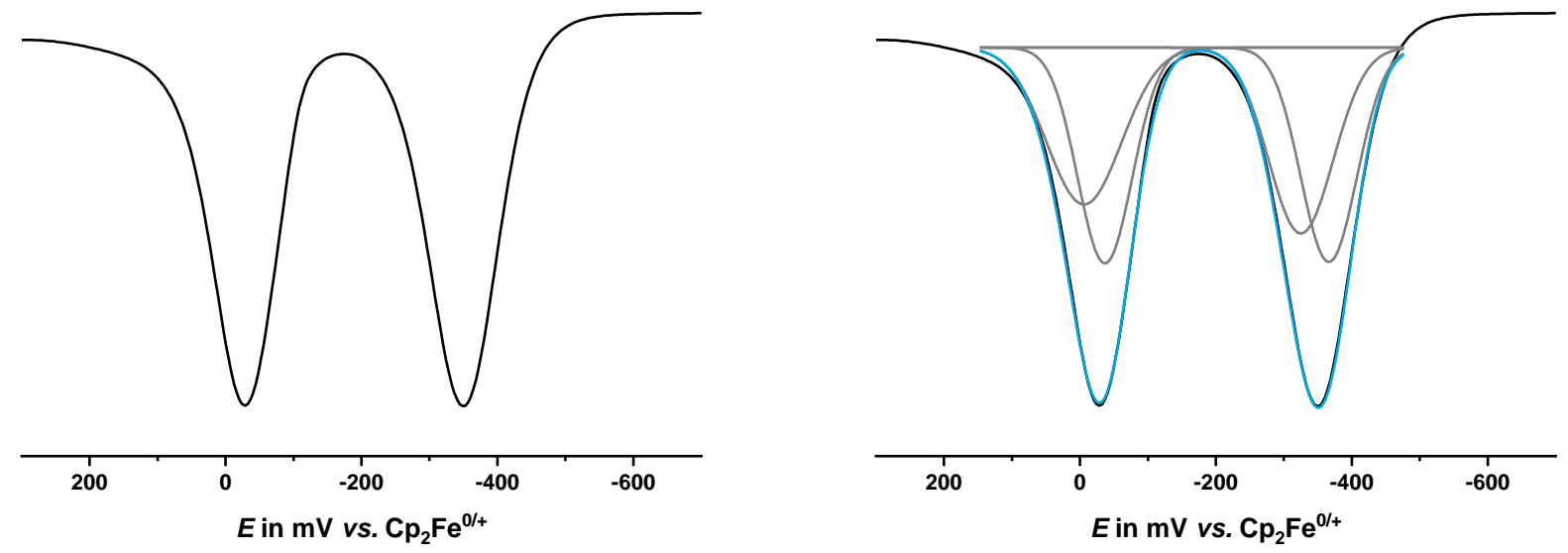

Figure S40. Square wave voltammogram of macrocycle 2-BT (left) with deconvolution (right), measured in $\mathrm{CH}_{2} \mathrm{Cl}_{2} /{ }^{n} \mathrm{Bu}_{4} \mathrm{NBAr}^{\mathrm{F} 24}$. 


\section{Compound 2-BTO:}
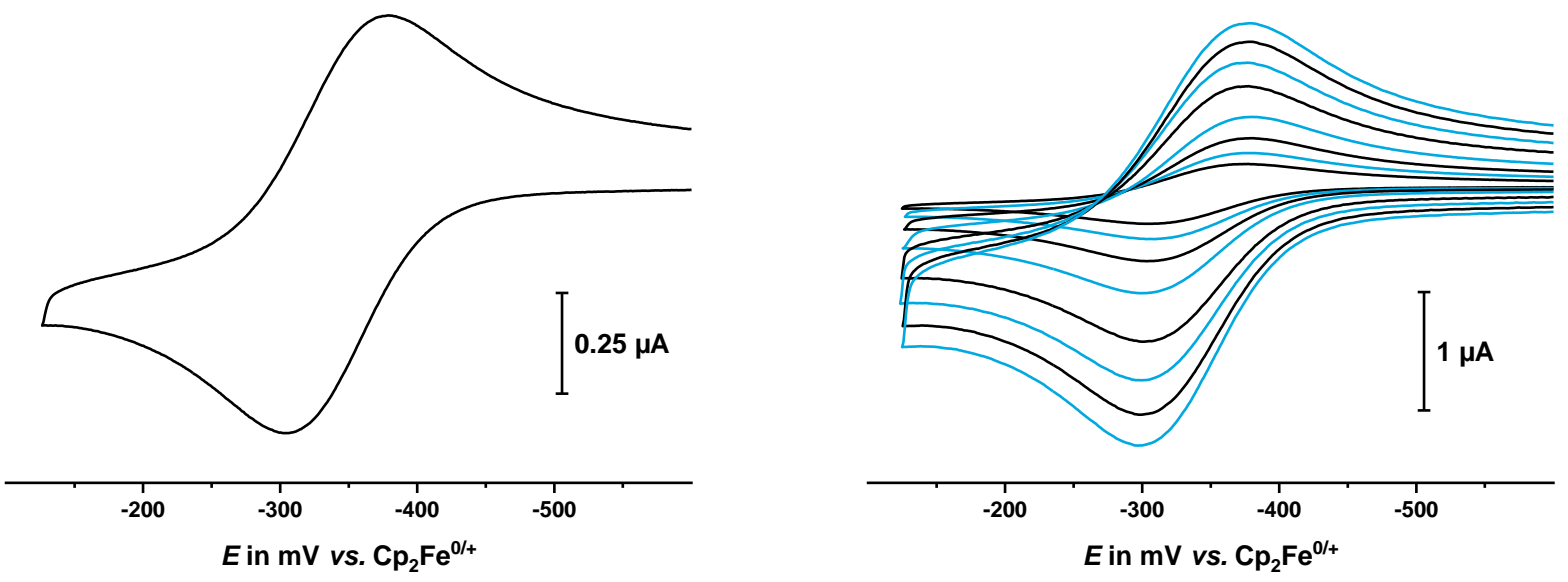

Figure S41. Cyclic voltammograms for the first composite wave of macrocycle 2-BTO at $v=100 \mathrm{mV} / \mathrm{s}$ (left) and at $v=25,50,100,200,400,600,800,1000 \mathrm{mV} / \mathrm{s}$ (right) in $\mathrm{CH}_{2} \mathrm{Cl}_{2} /{ }^{n} \mathrm{Bu}_{4} \mathrm{NBAr}^{\mathrm{F} 24}$.
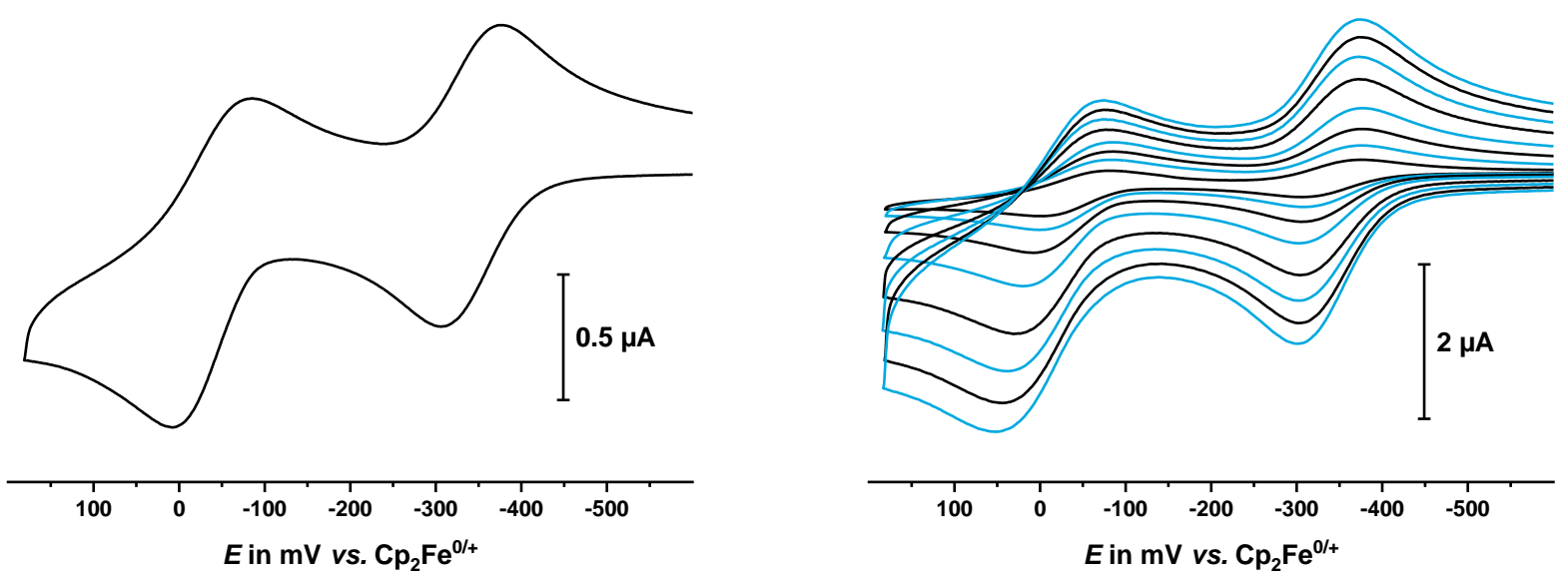

Figure S42. Cyclic voltammograms for the first two composite waves of macrocycle 2-BTO at $v=100 \mathrm{mV} / \mathrm{s}$ (left) and at $v=25,50,100,200,400,600,800,1000 \mathrm{mV} / \mathrm{s}$ (right) in $\mathrm{CH}_{2} \mathrm{Cl}_{2} /{ }^{n} \mathrm{Bu}_{4} \mathrm{NBAr}^{\mathrm{F} 24}$. 
Table S7. Data of the cyclovoltammetric measurements for macrocycle 2-BTO at different scan rates, measured in $\mathrm{CH}_{2} \mathrm{Cl}_{2} /{ }^{n} \mathrm{Bu}_{4} \mathrm{NBAr}^{\mathrm{F} 24}$.

\begin{tabular}{|c|c|c|c|c|c|}
\hline $\begin{array}{c}v \\
\text { in } \mathrm{mV} / \mathrm{s}\end{array}$ & $\begin{array}{l}E_{1 / 2}{ }^{0 / 2+} \\
\text { in } \mathbf{~} \mathbf{V}\end{array}$ & $\begin{array}{l}\Delta E_{\mathrm{p}}^{0 / 2+} \\
\text { in } \mathbf{m V}\end{array}$ & $\begin{array}{l}E_{1 / 2}{ }^{2+4+} \\
\text { in } \mathbf{m V}\end{array}$ & $\begin{array}{l}\Delta E_{\mathrm{p}}^{2+/ 4+} \\
\text { in } \mathrm{mV}\end{array}$ & $\begin{array}{c}\Delta E \\
\text { in } \mathrm{mV}\end{array}$ \\
\hline 25 & -340 & 71 & -41 & 82 & 382 \\
\hline 50 & -342 & 68 & -42 & 85 & 384 \\
\hline 100 & -341 & 69 & -38 & 93 & 380 \\
\hline 200 & -340 & 75 & -33 & 106 & 372 \\
\hline 400 & -338 & 69 & -23 & 107 & 361 \\
\hline 600 & -337 & 70 & -19 & 112 & 356 \\
\hline 800 & -338 & 73 & -16 & 120 & 354 \\
\hline 1000 & -337 & 73 & -11 & 127 & 348 \\
\hline
\end{tabular}
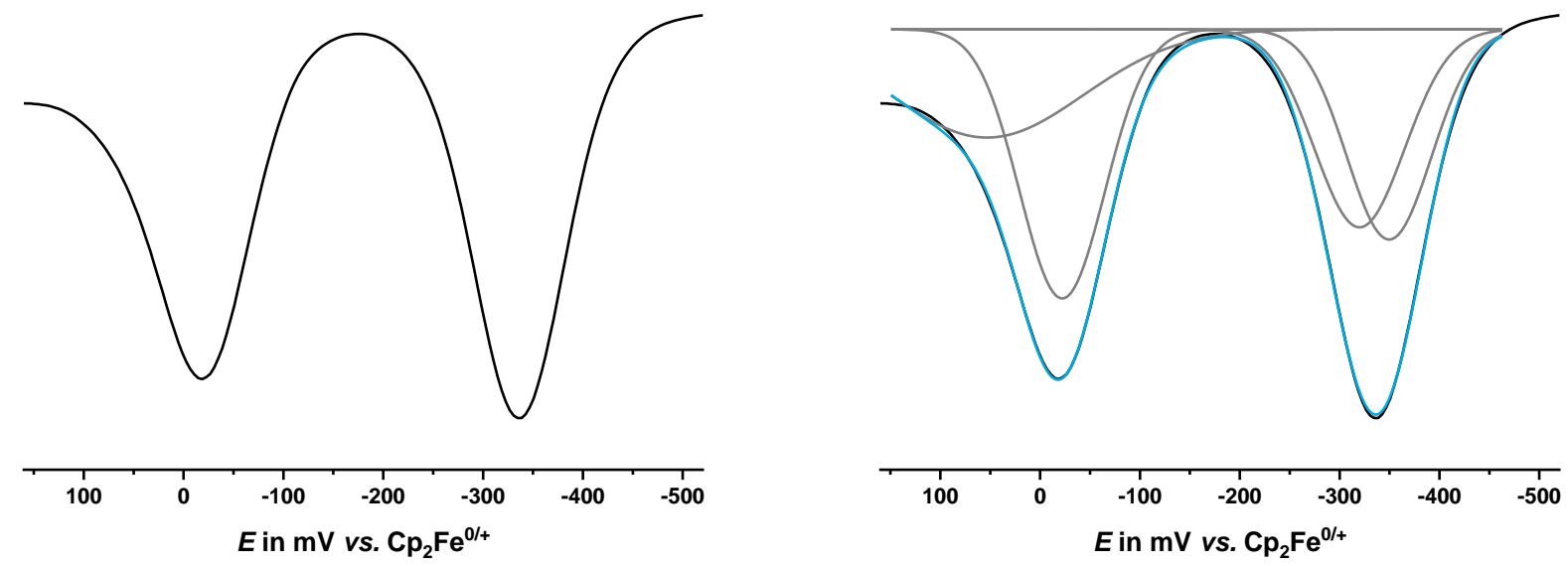

Figure S43. Square wave voltammogram of macrocycle 2-BTO (left) with deconvolution (right), measured in $\mathrm{CH}_{2} \mathrm{Cl}_{2} /{ }^{n} \mathrm{Bu}_{4} \mathrm{NBAr}^{\mathrm{F} 24}$. 


\section{Compound 2-BTE:}
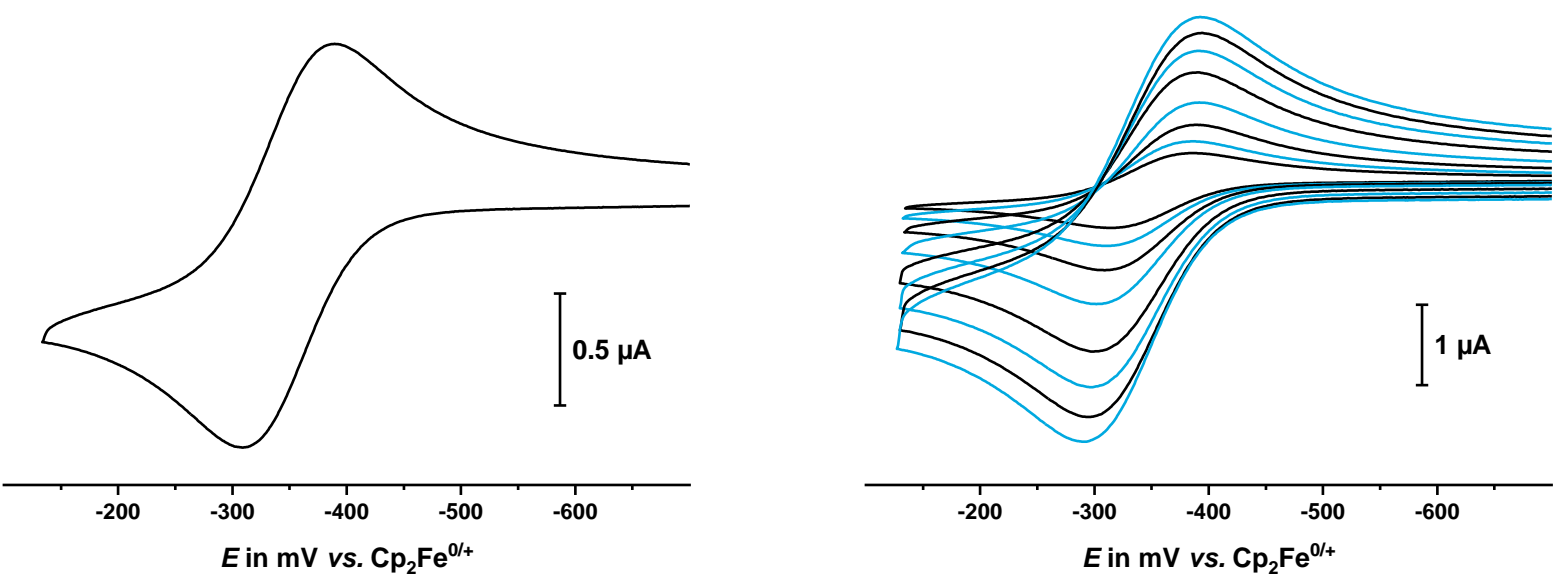

Figure S44. Cyclic voltammograms for the first composite wave of macrocycle 2-BTE at $v=100 \mathrm{mV} / \mathrm{s}$ (left) and at $v=25,50,100,200,400,600,800,1000 \mathrm{mV} / \mathrm{s}$ (right) in $\mathrm{CH}_{2} \mathrm{Cl}_{2}{ }^{n} \mathrm{Bu}_{4} \mathrm{NBAr}^{\mathrm{F} 24}$.
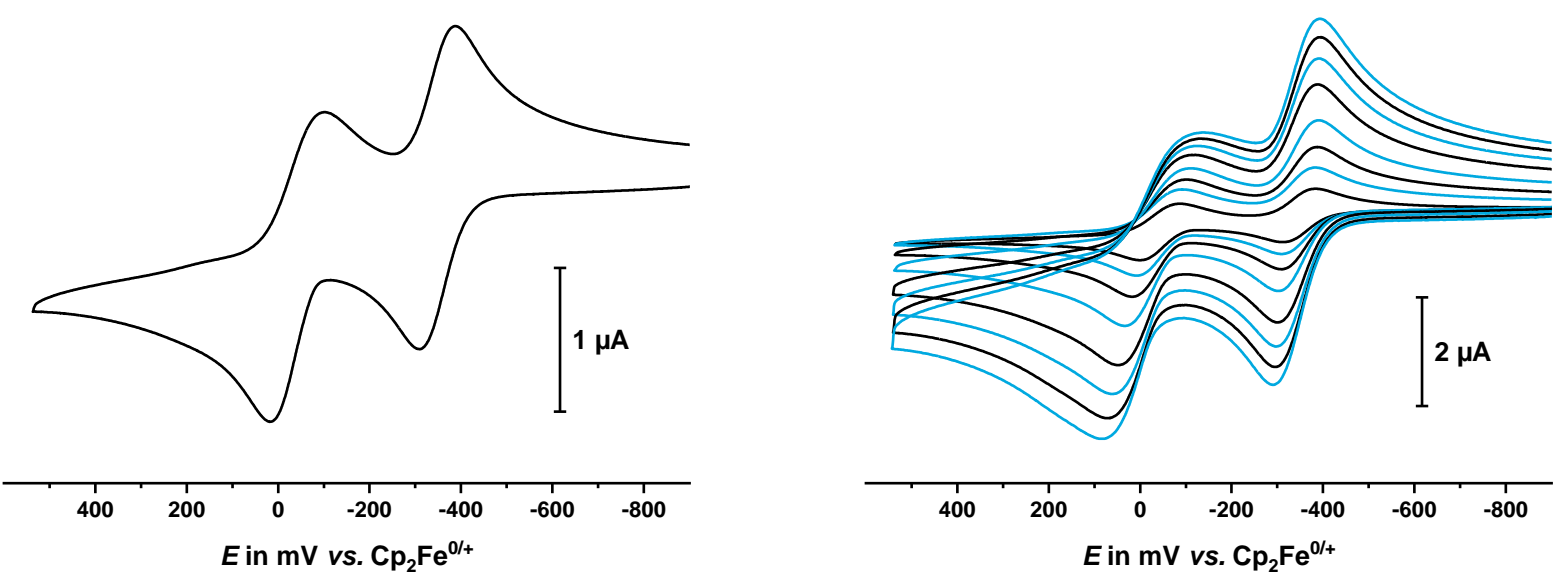

Figure S45. Cyclic voltammograms for the first two composite waves of macrocycle 2-BTE at $v=100 \mathrm{mV} / \mathrm{s}$ (left) and at $v=25,50,100,200,400,600,800,1000 \mathrm{mV} / \mathrm{s}$ (right) in $\mathrm{CH}_{2} \mathrm{Cl}_{2} /{ }^{n} \mathrm{Bu}_{4} \mathrm{NBAr}^{\mathrm{F} 24}$. 
Table S8. Data of the cyclovoltammetric measurements for macrocycle 2-BTE at different scan rates, measured in $\mathrm{CH}_{2} \mathrm{Cl}_{2} /{ }^{n} \mathrm{Bu}_{4} \mathrm{NBAr}^{\mathrm{F} 24}$.

\begin{tabular}{|c|c|c|c|c|c|}
\hline $\begin{array}{c}v \\
\text { in } \mathrm{mV} / \mathrm{s}\end{array}$ & $\begin{array}{l}E_{1 / 2}^{0 / 2+} \\
\text { in } \mathrm{mV}\end{array}$ & $\begin{array}{l}\Delta E_{\mathrm{p}}^{0 / 2+} \\
\text { in } \mathrm{mV}\end{array}$ & $\begin{array}{l}E_{1 / 2}^{2+/ 4+} \\
\text { in } \mathrm{mV}\end{array}$ & $\begin{array}{l}\Delta E_{\mathrm{p}}^{2+/ 4+} \\
\text { in } \mathrm{mV}\end{array}$ & $\begin{array}{c}\Delta E \\
\text { in } \mathrm{mV}\end{array}$ \\
\hline 25 & -350 & 71 & -43 & 88 & 306 \\
\hline 50 & -348 & 78 & -42 & 100 & 306 \\
\hline 100 & -349 & 81 & -41 & 119 & 306 \\
\hline 200 & -347 & 90 & -38 & 144 & 309 \\
\hline 400 & -345 & 90 & -35 & 166 & 310 \\
\hline 600 & -344 & 94 & -33 & 189 & 311 \\
\hline 800 & -344 & 99 & -31 & 206 & 312 \\
\hline 1000 & -342 & 101 & -27 & 222 & 316 \\
\hline
\end{tabular}
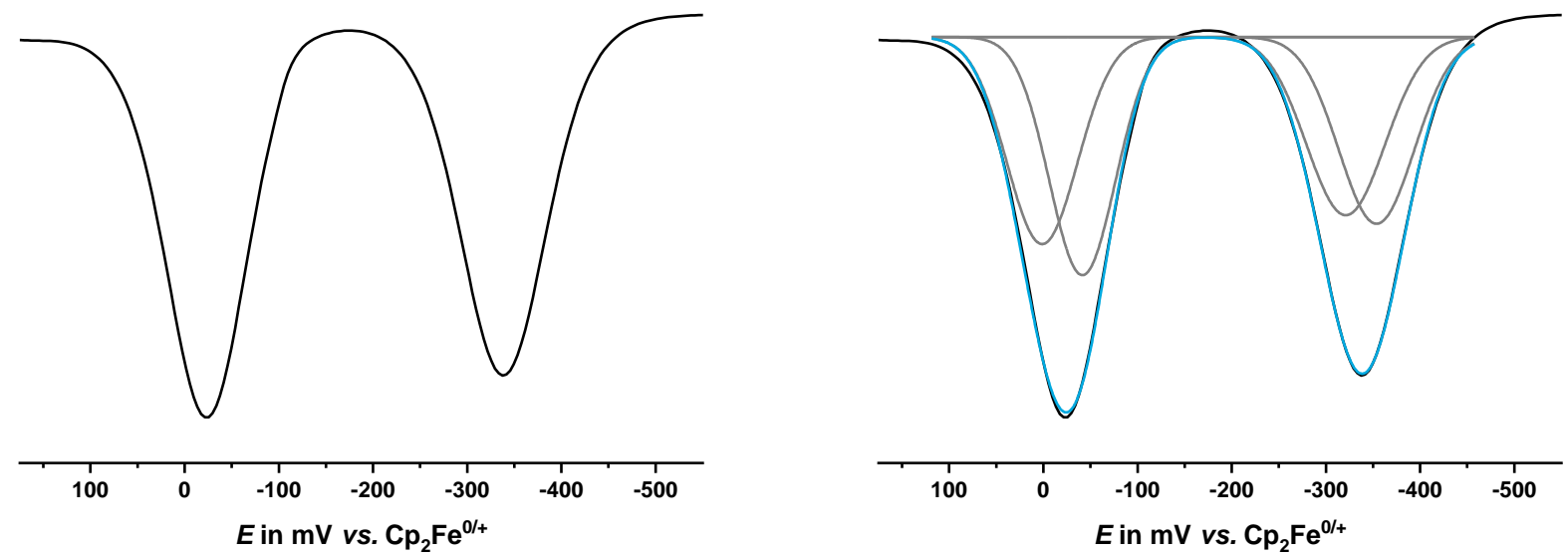

Figure S46. Square wave voltammogram of macrocycle 2-BTE (left) with deconvolution (right), measured in $\mathrm{CH}_{2} \mathrm{Cl}_{2} /{ }^{n} \mathrm{Bu}_{4} \mathrm{NBAr}^{\mathrm{F} 24}$. 


\section{Compound 2-BN:}
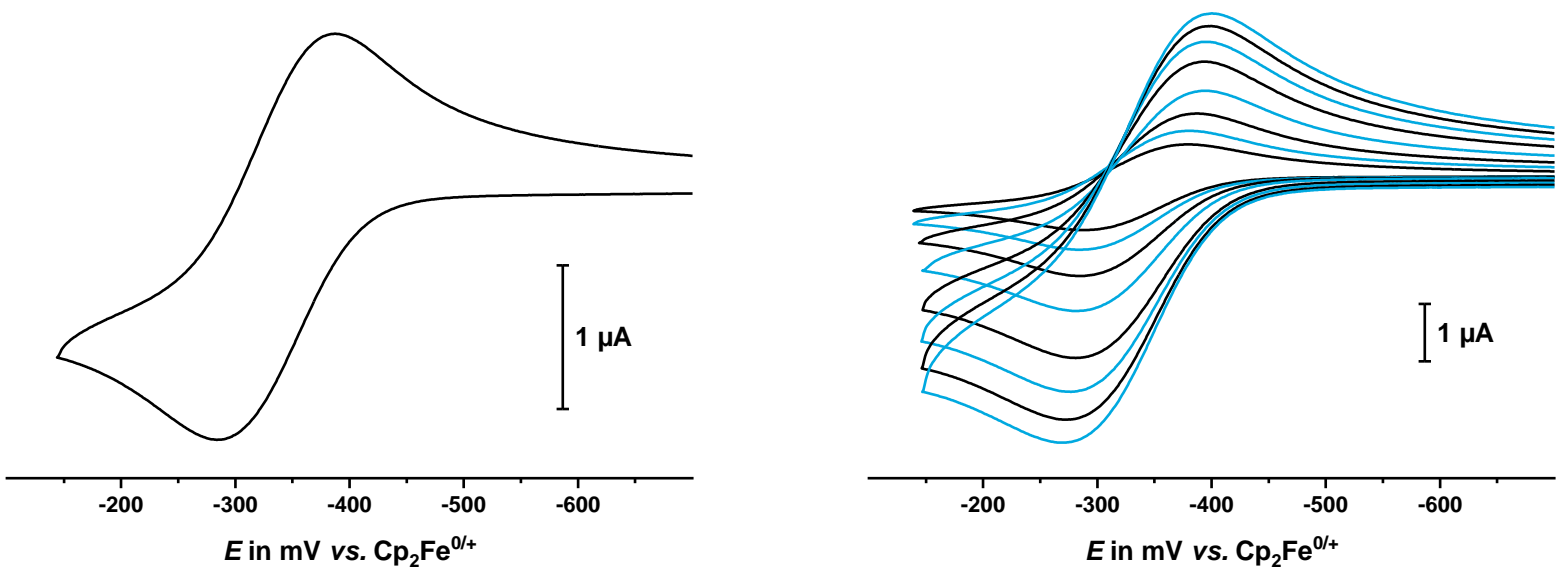

Figure S47. Cyclic voltammograms for the first composite wave of macrocycle 2-BN at $v=100 \mathrm{mV} / \mathrm{s}$ (left) and at $v=25,50,100,200,400,600,800,1000 \mathrm{mV} / \mathrm{s}$ (right) in $\mathrm{CH}_{2} \mathrm{Cl}_{2} /{ }^{n} \mathrm{Bu}_{4} \mathrm{NBAr}^{\mathrm{F2}}$.
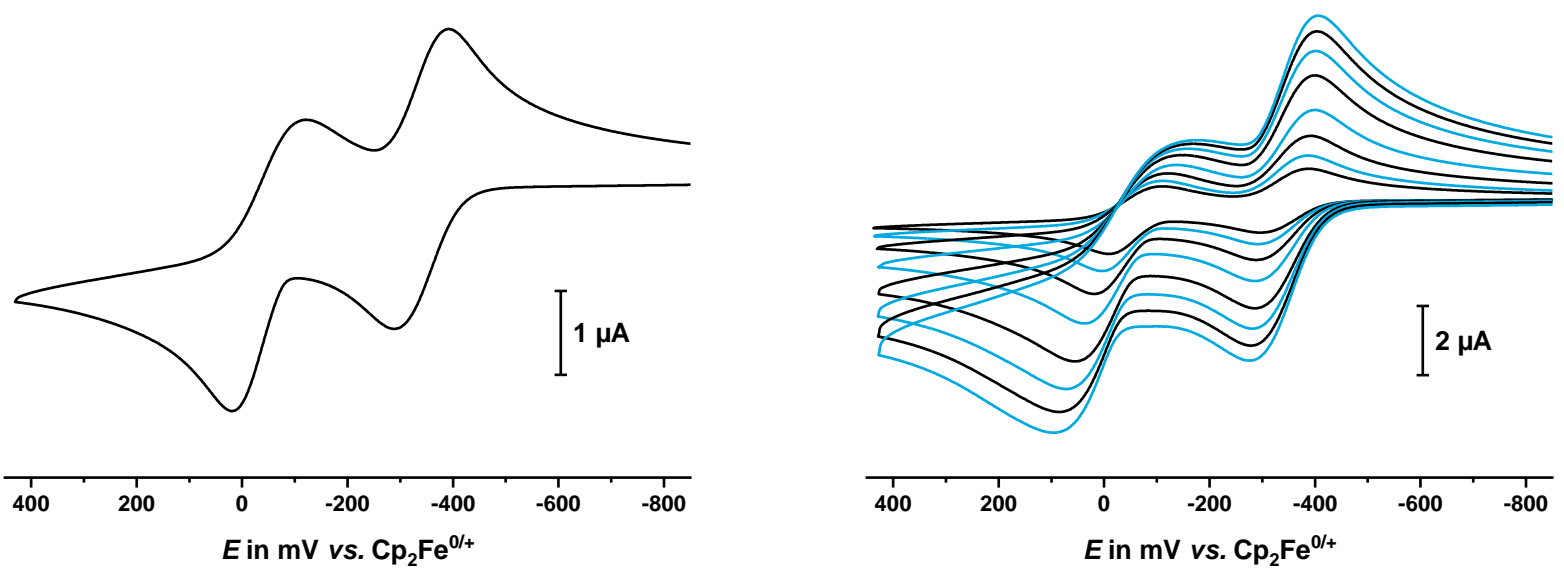

Figure S48. Cyclic voltammograms for the first two composite waves of macrocycle 2-BN at $v=100 \mathrm{mV} / \mathrm{s}$ (left) and at $v=25,50,100,200,400,600,800,1000 \mathrm{mV} / \mathrm{s}$ (right) in $\mathrm{CH}_{2} \mathrm{Cl}_{2} /{ }^{n} \mathrm{Bu}_{4} \mathrm{NBAr}^{\mathrm{F} 24}$.
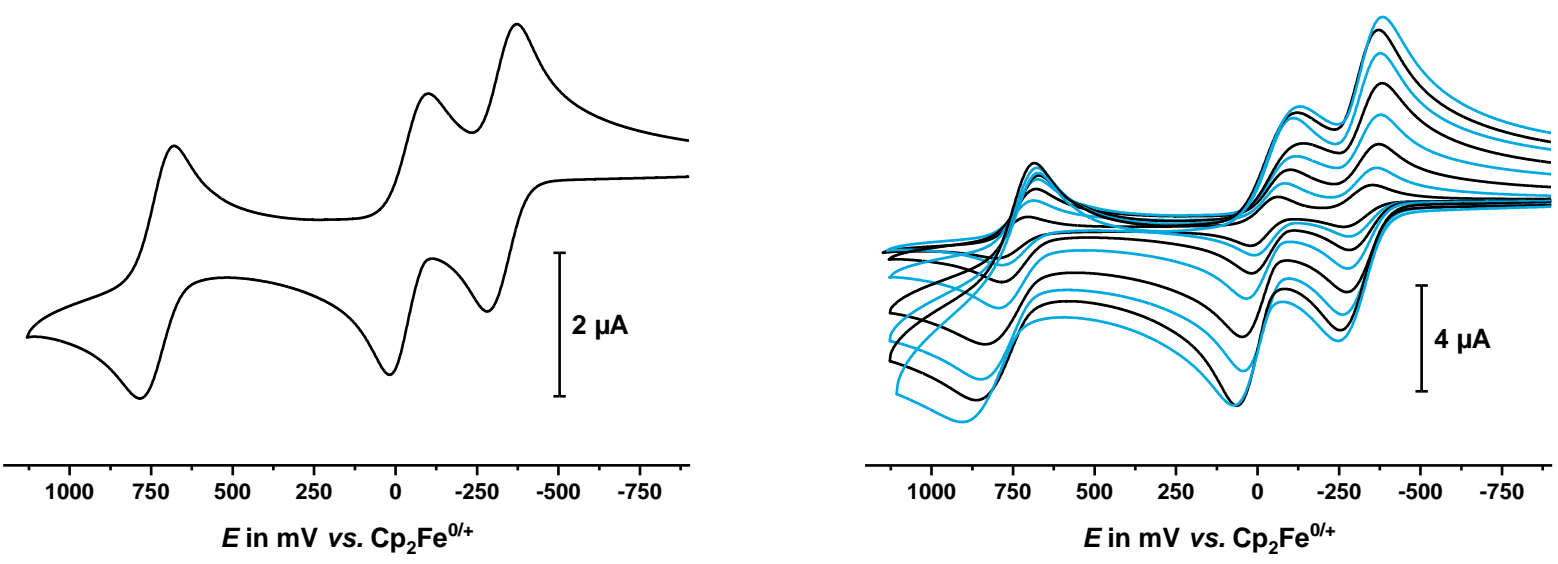

Figure S49. Cyclic voltammograms for the first three composite waves of macrocycle 2-BN at $v=100 \mathrm{mV} / \mathrm{s}$ (left) and at $v=25,50,100,200,400,600,800,1000 \mathrm{mV} / \mathrm{s}$ (right) in $\mathrm{CH}_{2} \mathrm{Cl}_{2} /{ }^{n} \mathrm{Bu}_{4} \mathrm{NBAr}^{\mathrm{F} 24}$. 
Table S9. Data of the cyclovoltammetric measurements for macrocycle 2-BN at different scan rates, measured in $\mathrm{CH}_{2} \mathrm{Cl}_{2} /{ }^{n} \mathrm{Bu}_{4} \mathrm{NBAr}^{\mathrm{F} 24}$.

\begin{tabular}{|c|c|c|c|c|c|c|}
\hline $\begin{array}{c}v \\
\text { in } \mathrm{mV} / \mathrm{s}\end{array}$ & $\begin{array}{l}E_{1 / 2}{ }^{0 / 2+} \\
\text { in } \mathbf{~} \mathbf{V} \\
\end{array}$ & $\begin{array}{l}\Delta E_{\mathrm{p}}^{0 / 2+} \\
\text { in } \mathrm{mV} \\
\end{array}$ & $\begin{array}{l}E_{1 / 2}^{2+/ 4+} \\
\text { in } \mathrm{mV} \\
\end{array}$ & $\begin{array}{l}\Delta E_{\mathrm{p}}{ }^{++/ 4+} \\
\text { in } \mathrm{mV}\end{array}$ & $\begin{array}{c}E_{1 / 2}{ }^{4+6+6+} \\
\text { in } \mathrm{mV} \\
\end{array}$ & $\begin{array}{c}\Delta E_{\mathrm{p}}^{\mathbf{4 + / 6 +}+} \\
\text { in } \mathrm{mV} \\
\end{array}$ \\
\hline 25 & -319 & 92 & -47 & 102 & 752 & 99 \\
\hline 50 & -332 & 96 & -54 & 115 & 735 & 97 \\
\hline 100 & -336 & 103 & -51 & 140 & 733 & 104 \\
\hline 200 & -338 & 112 & -50 & 175 & 735 & 119 \\
\hline 400 & -337 & 113 & -46 & 204 & 755 & 164 \\
\hline 600 & -336 & 120 & -43 & 232 & 762 & 175 \\
\hline 800 & -335 & 126 & -42 & 256 & 774 & 179 \\
\hline 1000 & -335 & 132 & -41 & 271 & 784 & 182 \\
\hline
\end{tabular}
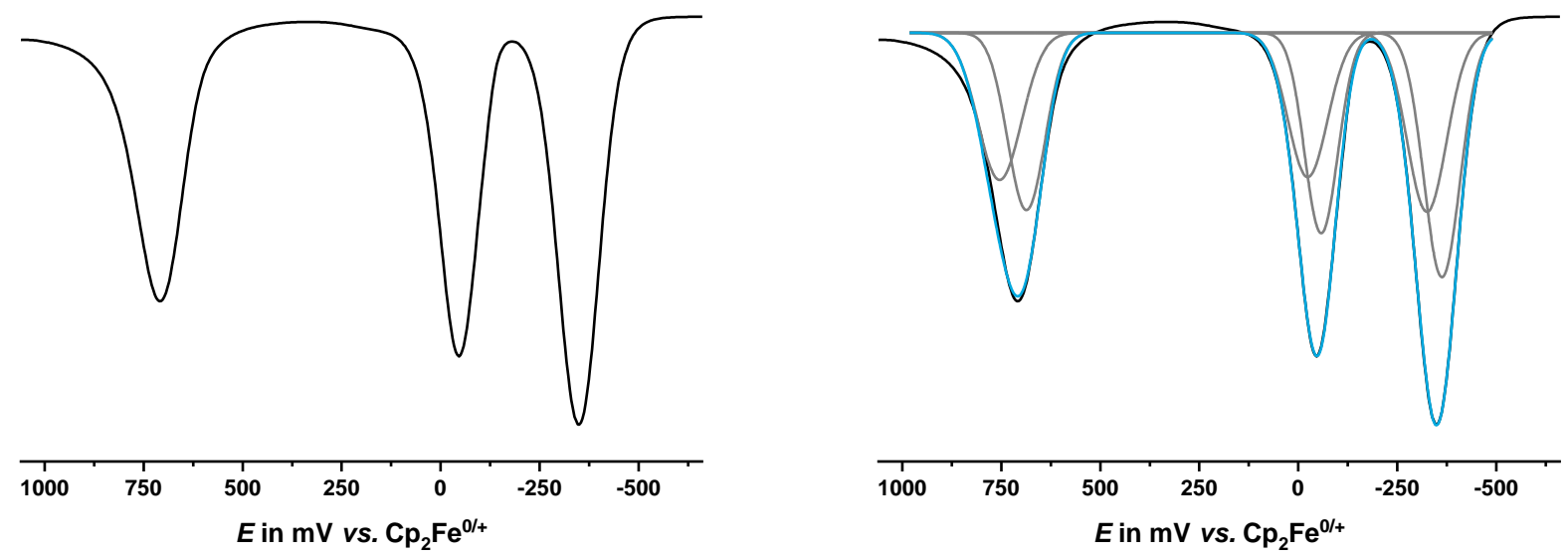

Figure S50. Square wave voltammogram of macrocycle 2-BN (left) with deconvolution (right), measured in $\mathrm{CH}_{2} \mathrm{Cl}_{2} /{ }^{n} \mathrm{Bu}_{4} \mathrm{NBAr}^{\mathrm{F} 24}$. 


\section{Compound 2-NB:}
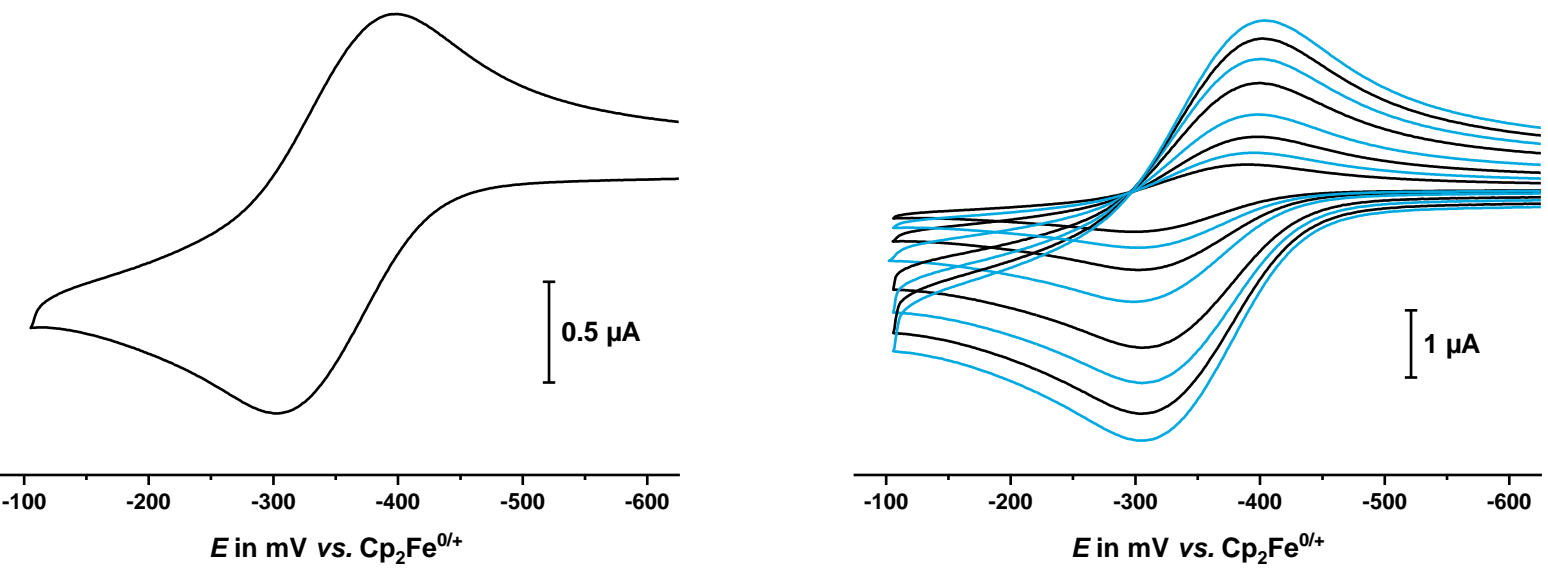

Figure S51. Cyclic voltammograms for the first composite wave of macrocycle 2-NB at $v=100 \mathrm{mV} / \mathrm{s}$ (left) and at $v=25,50,100,200,400,600,800,1000 \mathrm{mV} / \mathrm{s}$ (right) in $\mathrm{CH}_{2} \mathrm{Cl}_{2} /{ }^{n} \mathrm{Bu}_{4} \mathrm{NBAr}^{\mathrm{F} 24}$.
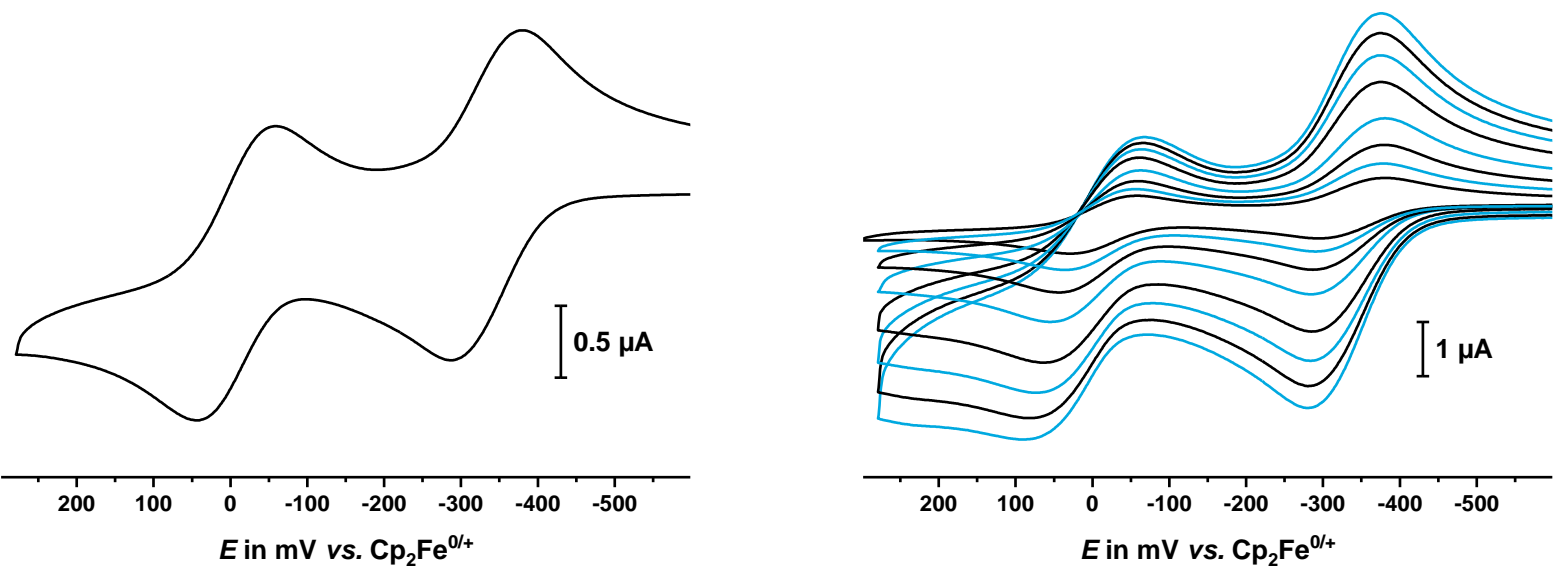

Figure S52. Cyclic voltammograms for the first two composite waves of macrocycle 2-NB at $v=100 \mathrm{mV} / \mathrm{s}$ (left) and at $v=25,50,100,200,400,600,800,1000 \mathrm{mV} / \mathrm{s}$ (right) in $\mathrm{CH}_{2} \mathrm{Cl}_{2} /{ }^{n} \mathrm{Bu}_{4} \mathrm{NBAr}^{\mathrm{F} 24}$.
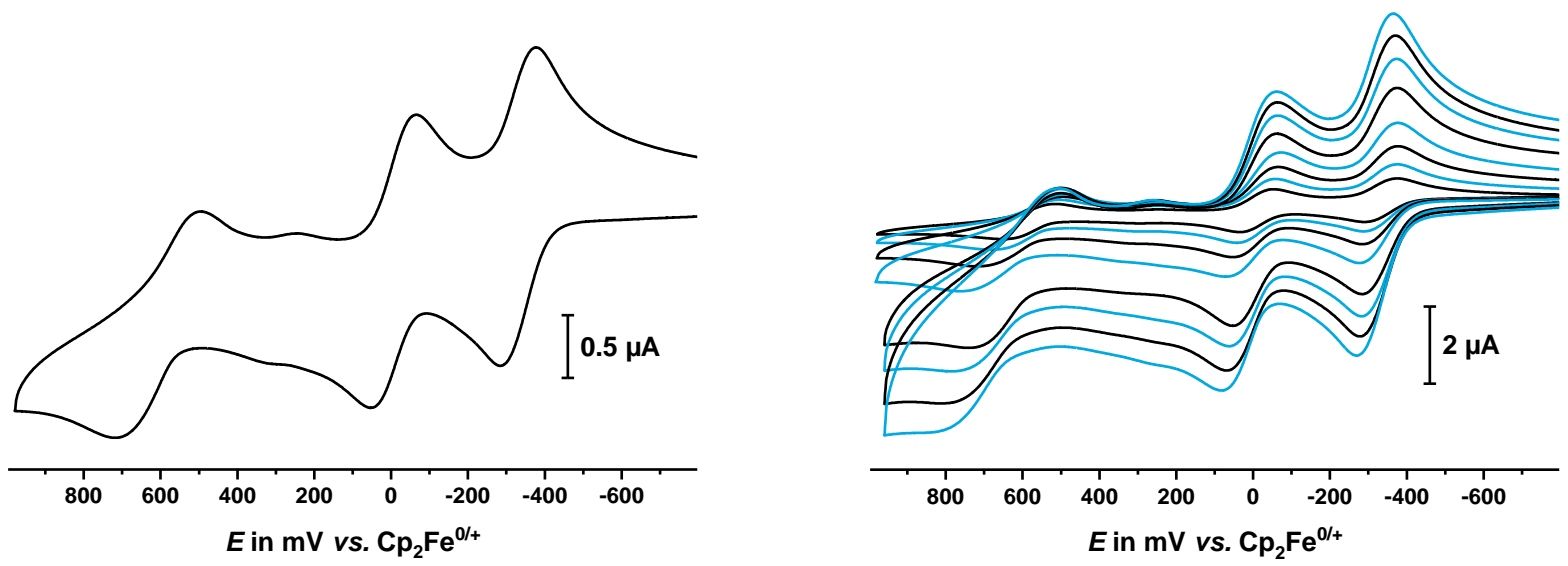

Figure S53. Cyclic voltammograms for the first two composite waves of macrocycle 2-NB at $v=100 \mathrm{mV} / \mathrm{s}$ (left) and at $v=25,50,100,200,400,600,800,1000 \mathrm{mV} / \mathrm{s}$ (right) in $\mathrm{CH}_{2} \mathrm{Cl}_{2} /{ }^{n} \mathrm{Bu}_{4} \mathrm{NBAr}^{\mathrm{F} 24}$. 
Table S10. Data of the cyclovoltammetric measurements for macrocycle 2-NB at different scan rates, measured in $\mathrm{CH}_{2} \mathrm{Cl}_{2} /{ }^{n} \mathrm{Bu}_{4} \mathrm{NBAr}^{\mathrm{F} 24}$.

\begin{tabular}{|c|c|c|c|c|c|c|}
\hline $\begin{array}{c}v \\
\text { in } \mathrm{mV} / \mathrm{s} \\
\end{array}$ & $\begin{array}{l}E_{1 / 2}{ }^{0 / 2+} \\
\text { in } \mathbf{m V} \\
\end{array}$ & $\begin{array}{l}\Delta E_{\mathrm{p}}{ }^{0 / 2+} \\
\text { in } \mathrm{mV} \\
\end{array}$ & $\begin{array}{c}E_{1 / 2}^{2+/ 4+} \\
\text { in } \mathrm{mV} \\
\end{array}$ & $\begin{array}{c}\Delta E_{\mathrm{p}}^{2+/ 4+} \\
\text { in } \mathrm{mV} \\
\end{array}$ & $\begin{array}{c}E_{1 / 2}{ }^{4+/ 6+} \\
\text { in } \mathrm{mV} \\
\end{array}$ & $\begin{array}{c}\Delta E_{\mathrm{p}}^{4+/ 6+} \\
\text { in } \mathrm{mV} \\
\end{array}$ \\
\hline 25 & -346 & 90 & -93 & 95 & 501 & 135 \\
\hline 50 & -349 & 93 & -10 & 94 & 593 & 175 \\
\hline 100 & -350 & 96 & -7 & 102 & 605 & 227 \\
\hline 200 & -348 & 99 & -1 & 117 & 627 & 271 \\
\hline 400 & -345 & 95 & 8 & 124 & 647 & 238 \\
\hline 600 & -345 & 95 & 13 & 138 & 668 & 284 \\
\hline 800 & -343 & 97 & 18 & 150 & 686 & 311 \\
\hline 1000 & -343 & 100 & 23 & 157 & 707 & 343 \\
\hline
\end{tabular}
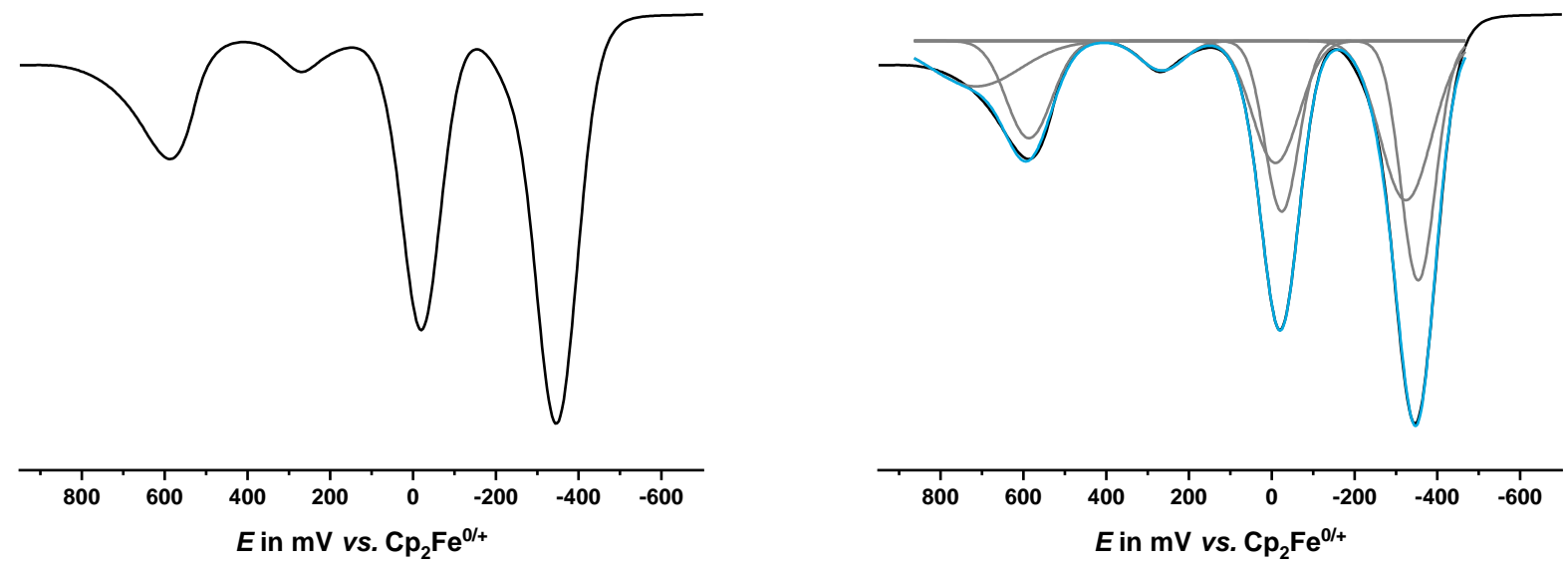

Figure S54. Square wave voltammogram of macrocycle 2-NB (left) with deconvolution (right), measured in $\left.\mathrm{CH}_{2} \mathrm{Cl}_{2}\right|^{n} \mathrm{Bu}_{4} \mathrm{NBAr}{ }^{\mathrm{F} 24}$. 


\section{Spectroelectrochemistry}

\section{Compound 2-BT:}
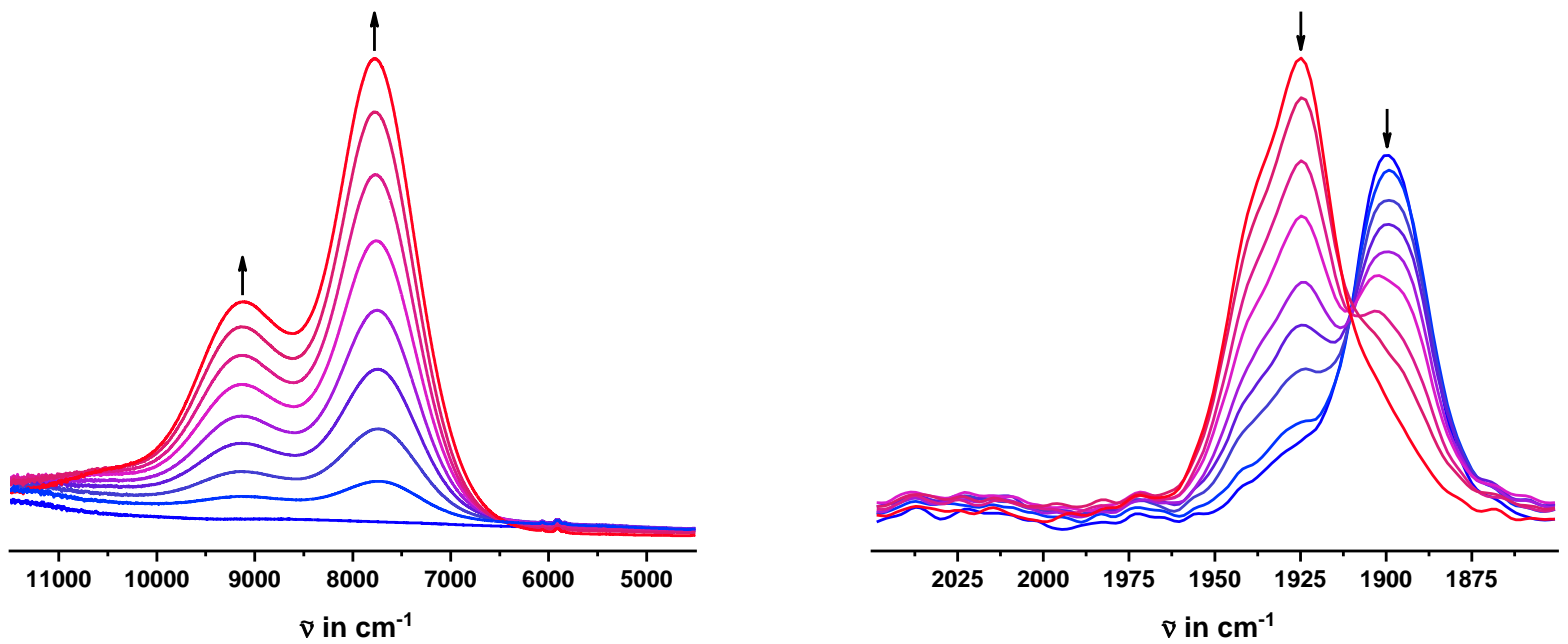

Figure S55. Changes in the IR spectra of 2-BT upon oxidation to 2-BT ${ }^{2+}$, measured in $\mathrm{CH}_{2} \mathrm{Cl}_{2} /{ }^{n} \mathrm{Bu}_{4} \mathrm{NPF}_{6}$.
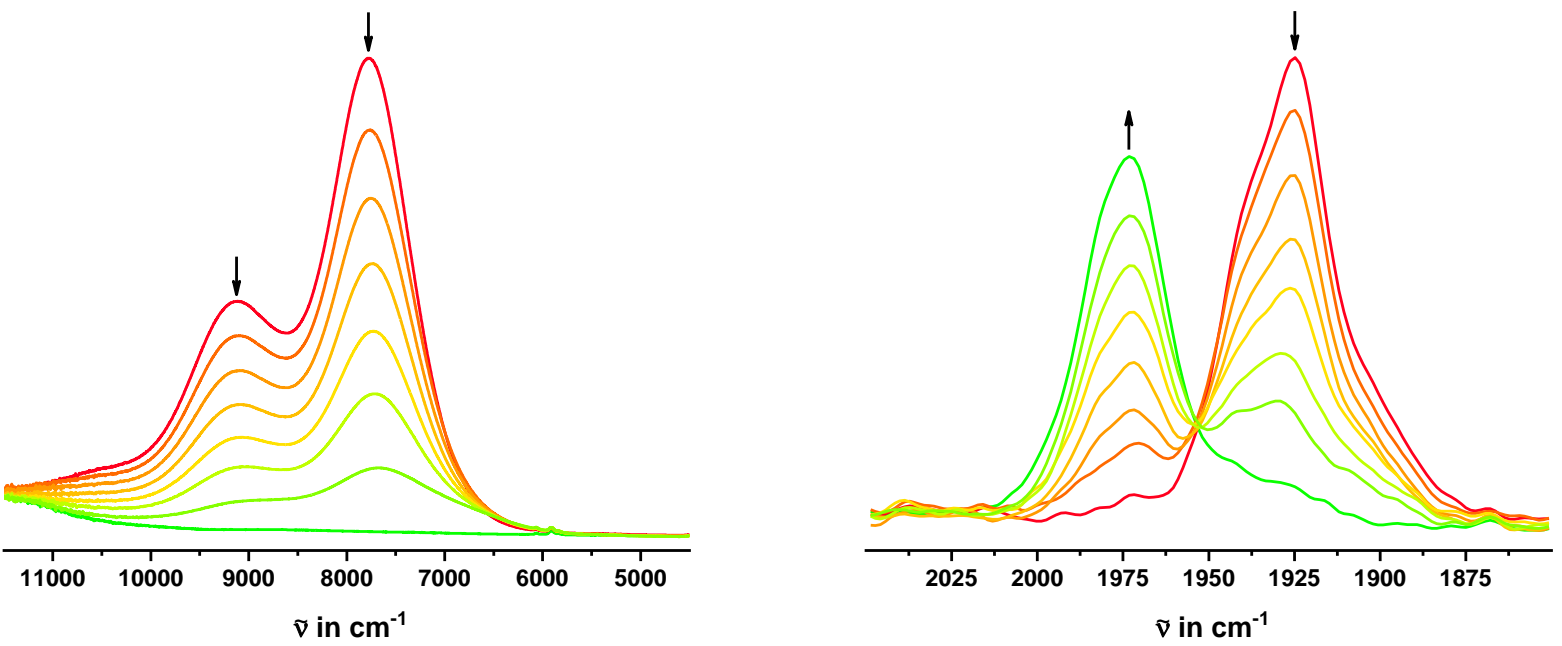

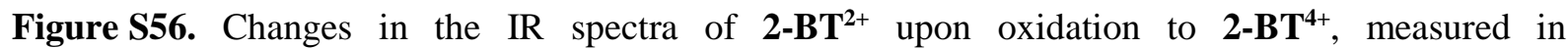
$\mathrm{CH}_{2} \mathrm{Cl}_{2} /{ }^{n} \mathrm{Bu}_{4} \mathrm{NPF}_{6}$.
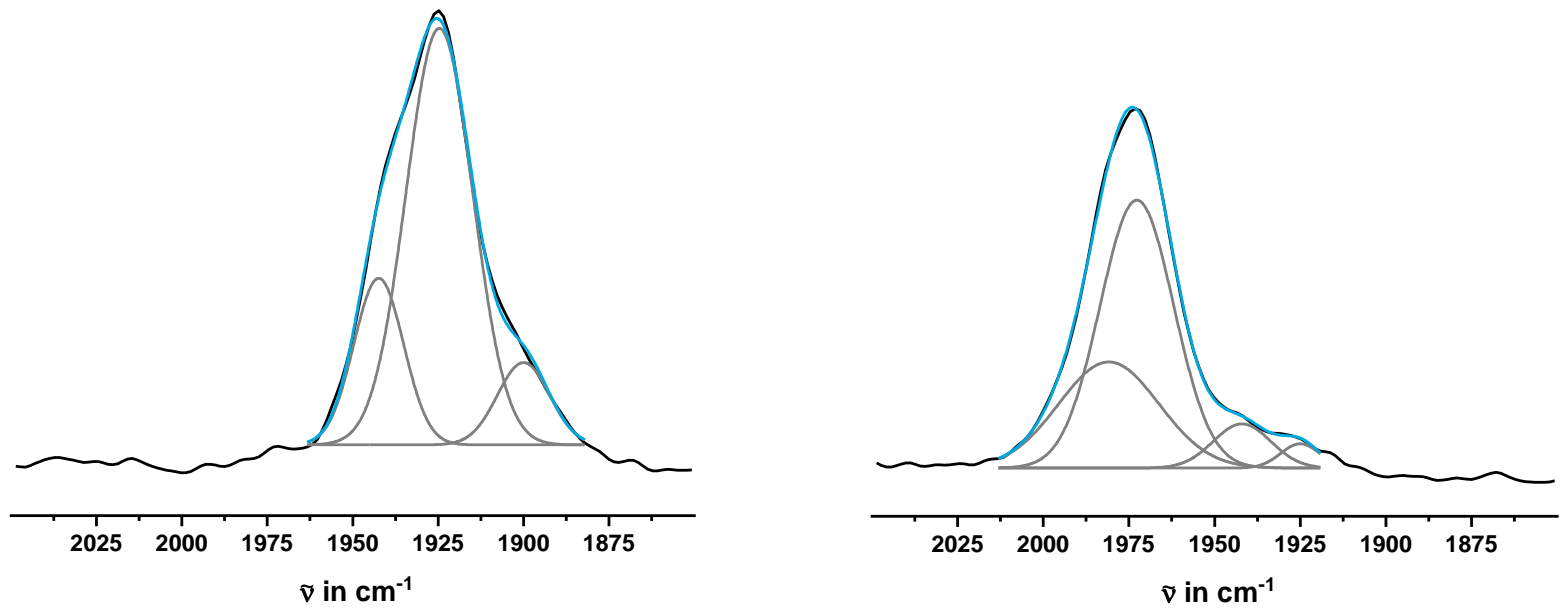

Figure S57. Deconvolutions of the Carbonyl bands of 2-BT ${ }^{2+}$ (left) and $\mathbf{2 - B T ^ { 4 + }}$ (right). 

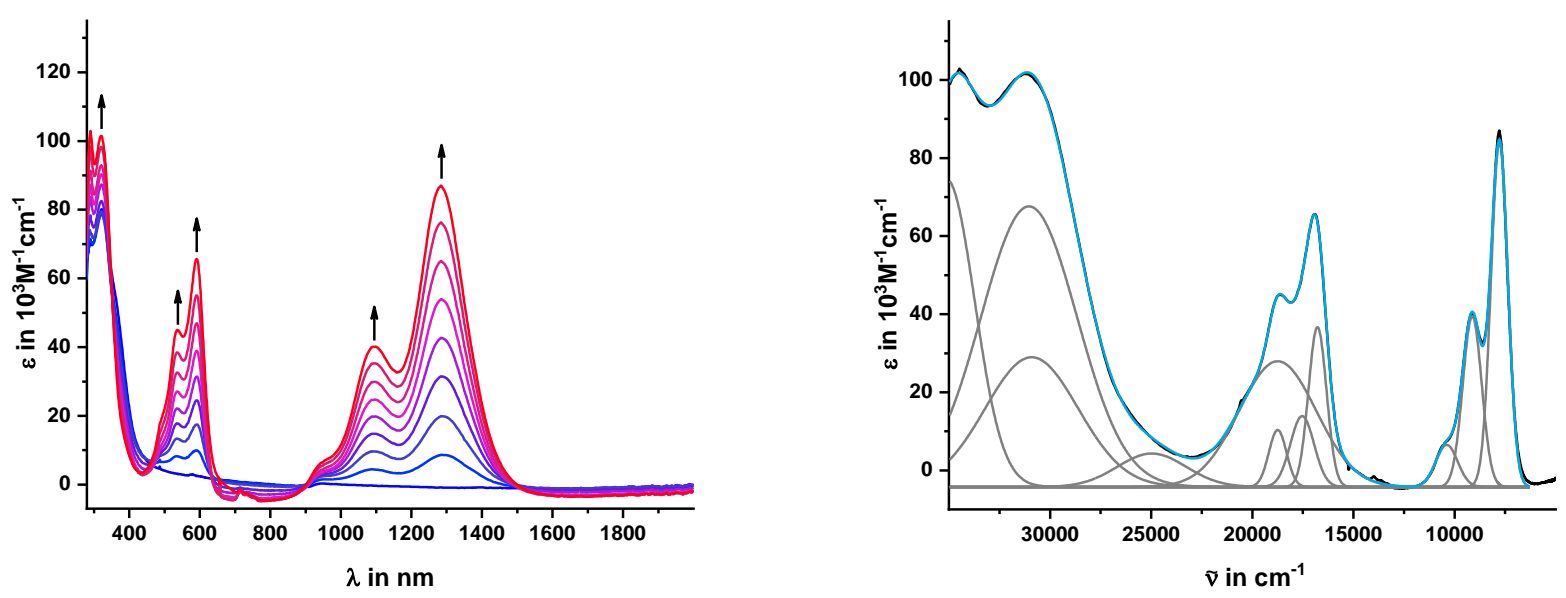

Figure S58. Changes in the UV/Vis/NIR spectra of 2-BT upon oxidation to 2-BT ${ }^{2+}$ (left) and deconvolution (right), measured in $\mathrm{CH}_{2} \mathrm{Cl}_{2} /{ }^{n} \mathrm{Bu}_{4} \mathrm{NPF}_{6}$.
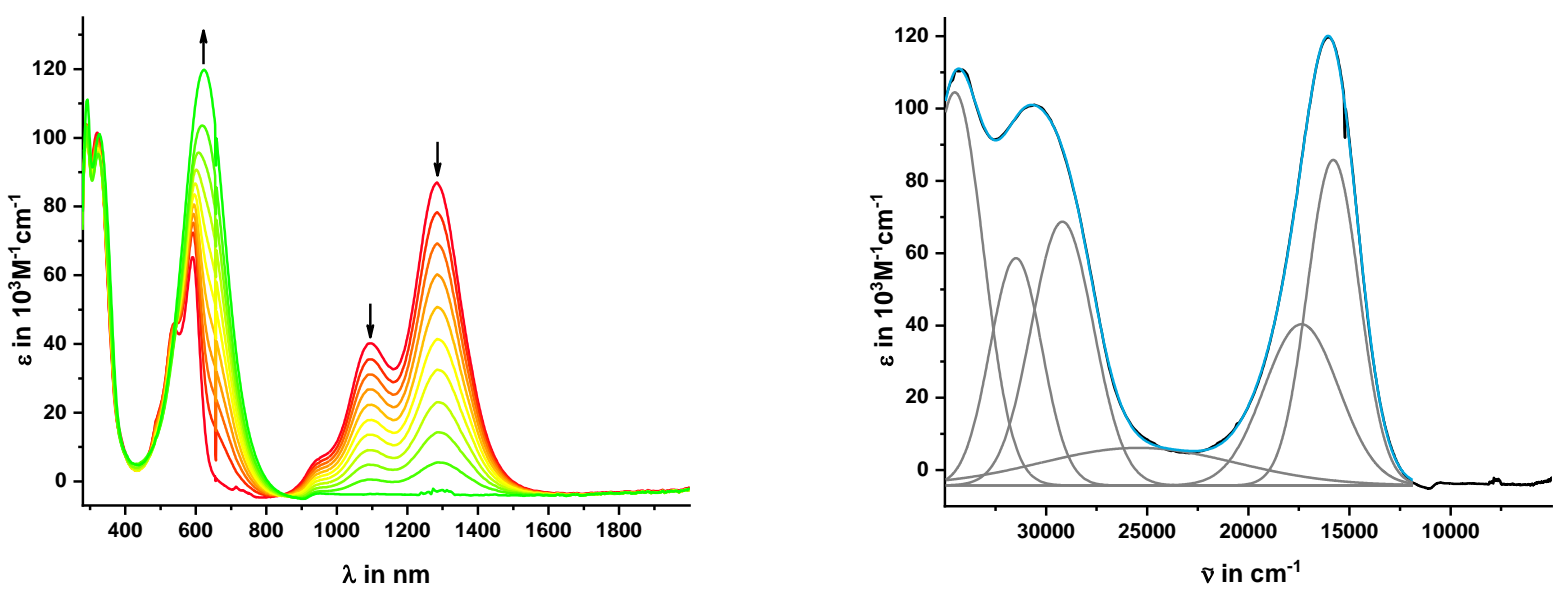

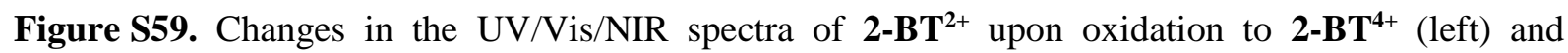
deconvolution (right), measured in $\mathrm{CH}_{2} \mathrm{Cl}_{2} /{ }^{n} \mathrm{Bu}_{4} \mathrm{NPF}_{6}$. 

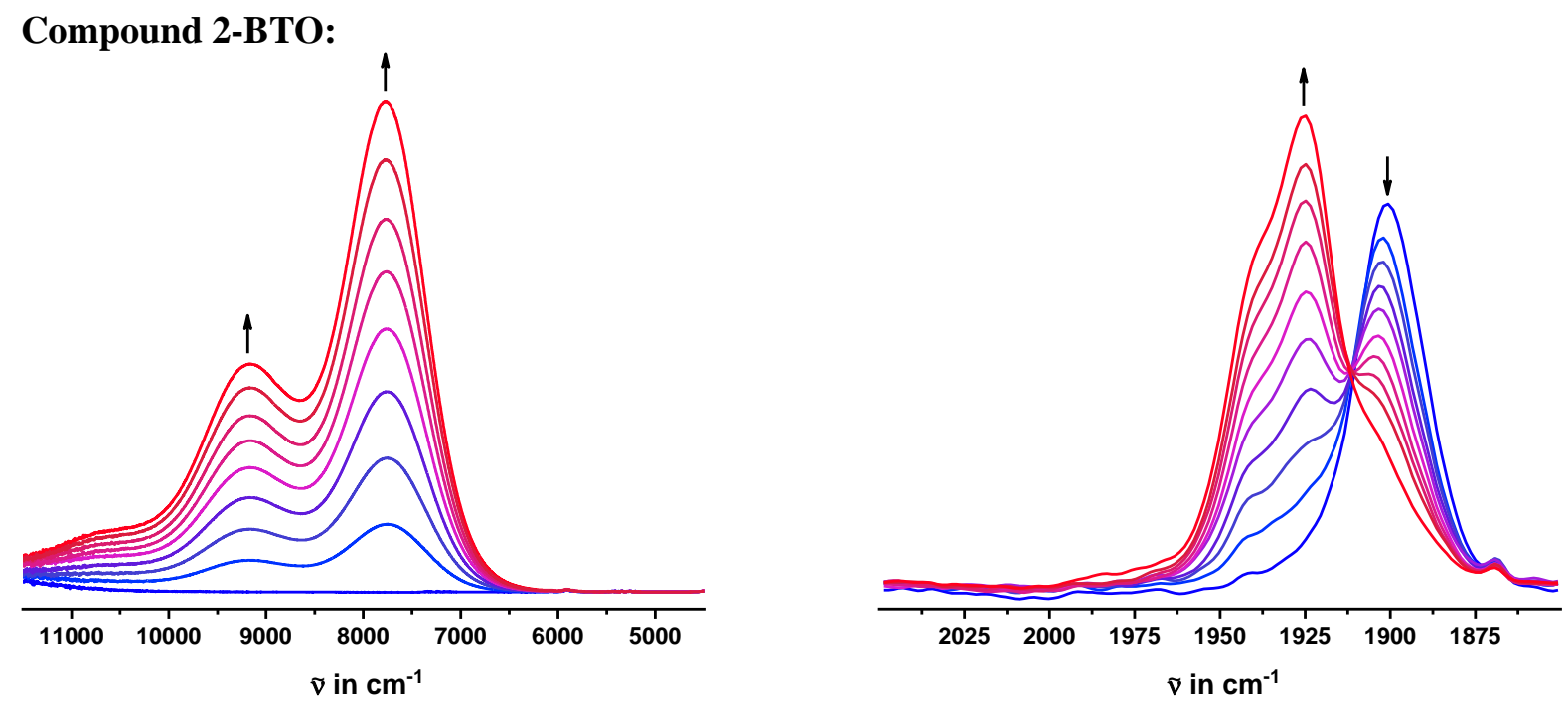

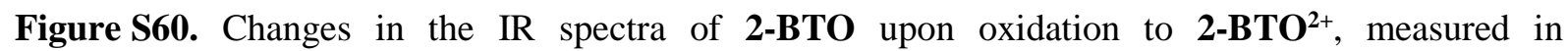
$\mathrm{CH}_{2} \mathrm{Cl}_{2} /{ }^{n} \mathrm{Bu}_{4} \mathrm{NPF}_{6}$.
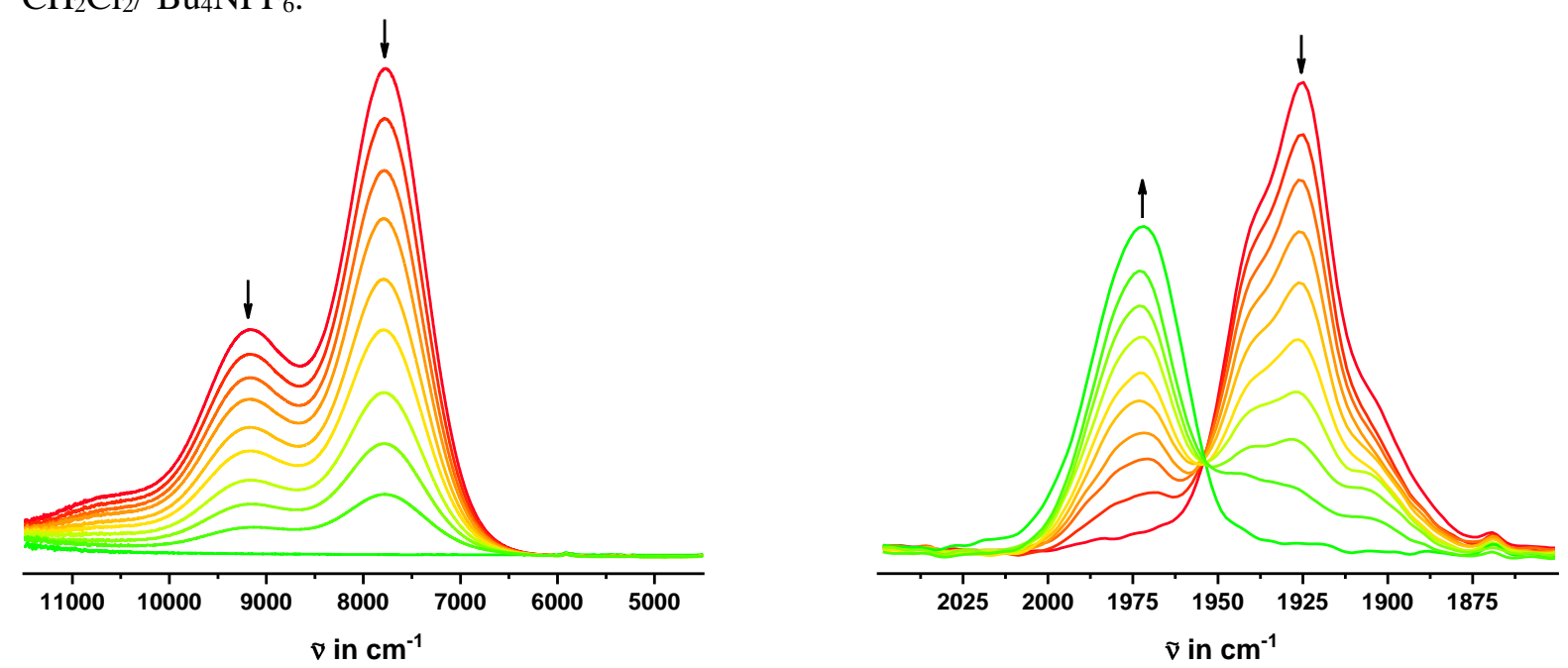

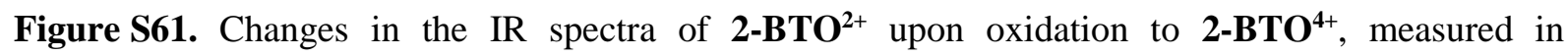
$\mathrm{CH}_{2} \mathrm{Cl}_{2} /{ }^{n} \mathrm{Bu}_{4} \mathrm{NPF}_{6}$.
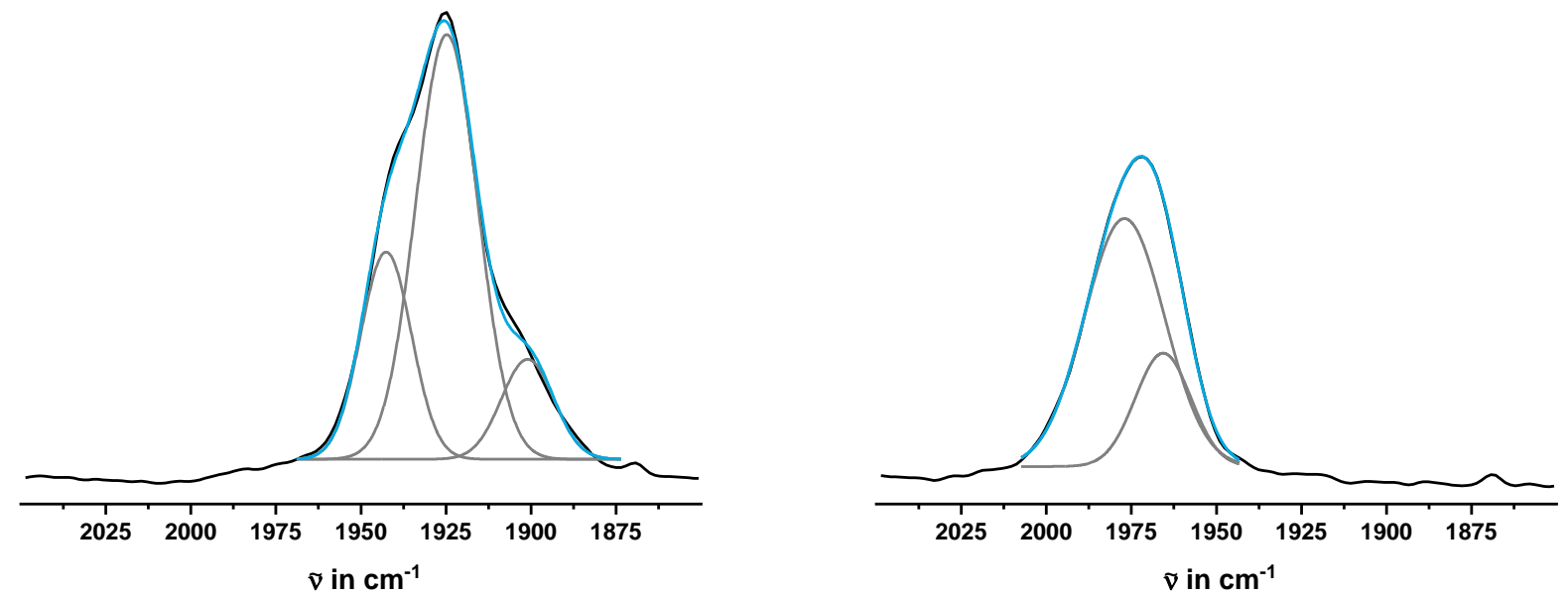

Figure S62. Deconvolutions of the Carbonyl bands of 2-BTO ${ }^{2+}$ (left) and 2-BTO ${ }^{4+}$ (right). 

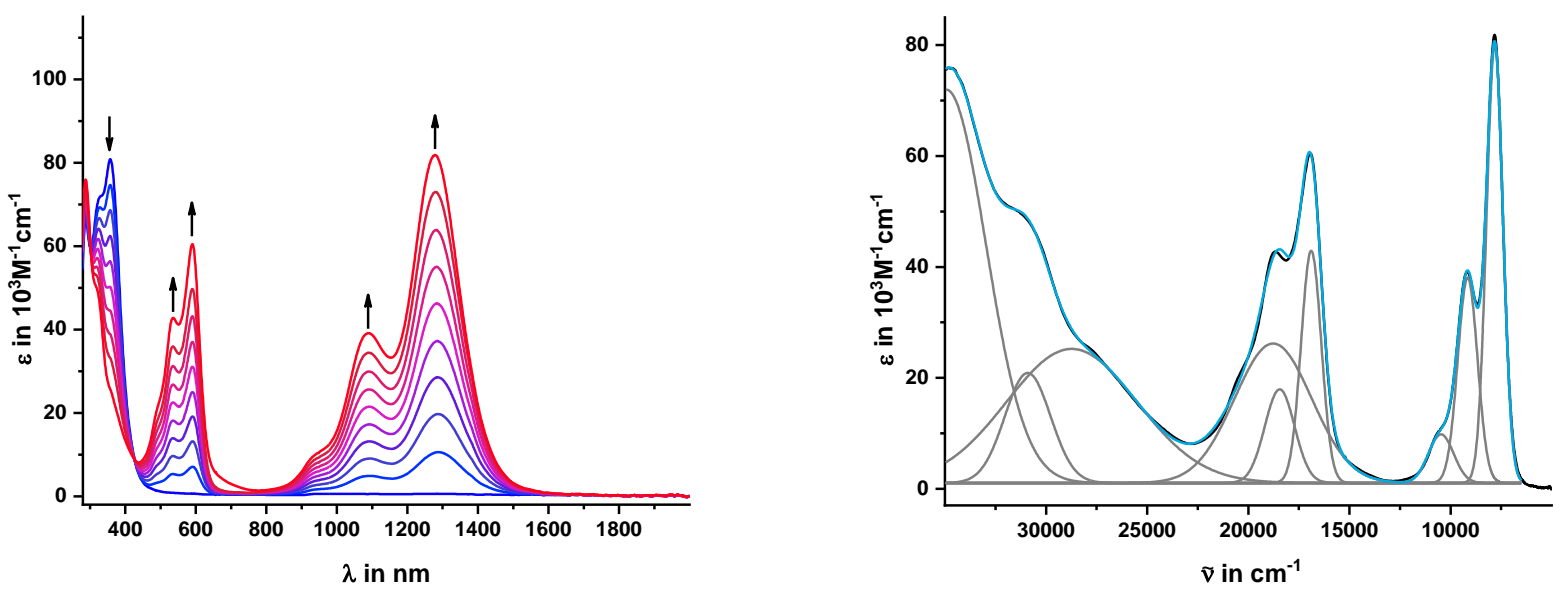

Figure S63. Changes in the UV/Vis/NIR spectra of 2-BTO upon oxidation to 2-BTO ${ }^{2+}$ (left) and deconvolution (right), measured in $\mathrm{CH}_{2} \mathrm{Cl}_{2} /{ }^{n} \mathrm{Bu}_{4} \mathrm{NPF}_{6}$.
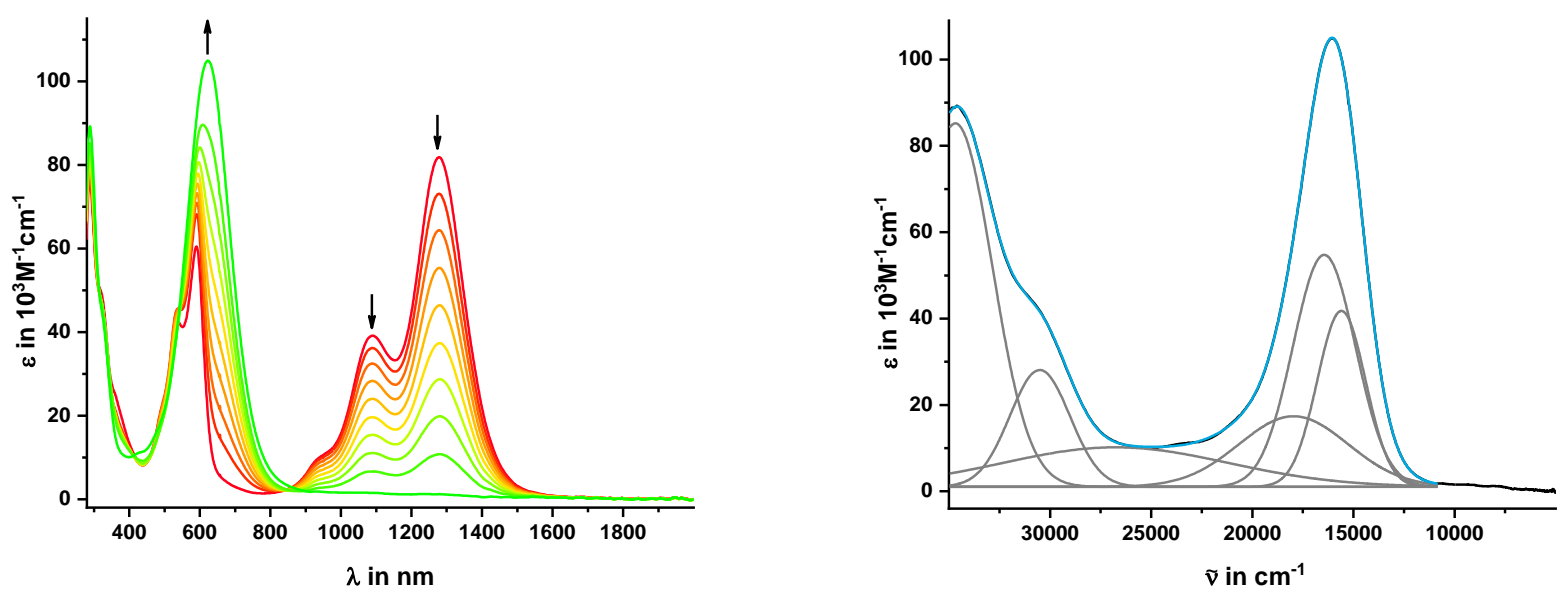

Figure S64. Changes in the UV/Vis/NIR spectra of 2-BTO ${ }^{2+}$ upon oxidation to 2-BTO ${ }^{4+}$ (left) and deconvolution (right), measured in $\mathrm{CH}_{2} \mathrm{Cl}_{2} /{ }^{n} \mathrm{Bu}_{4} \mathrm{NPF}_{6}$. 


\section{Compound 2-BTE:}
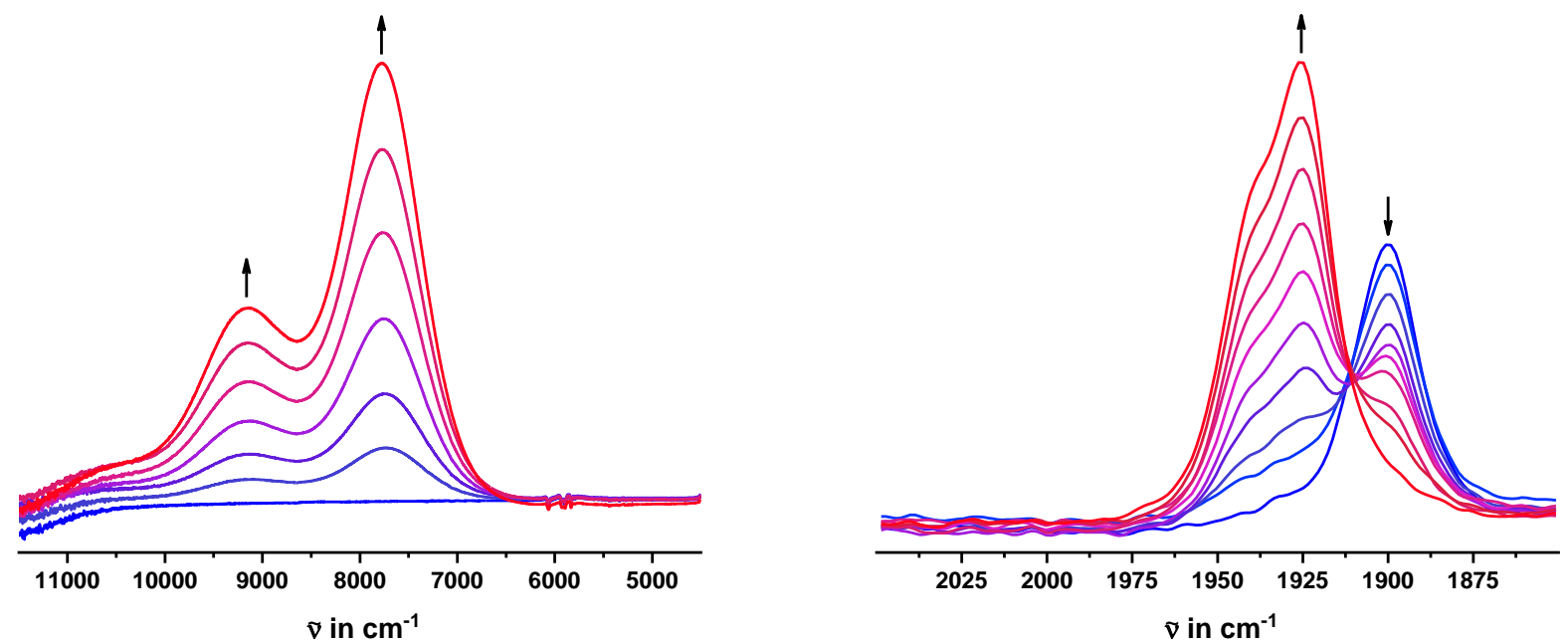

Figure S65. Changes in the IR spectra of 2-BTE upon oxidation to 2-BTE ${ }^{2+}$, measured in $\mathrm{CH}_{2} \mathrm{Cl}_{2} /{ }^{n} \mathrm{Bu}_{4} \mathrm{NPF}_{6}$.
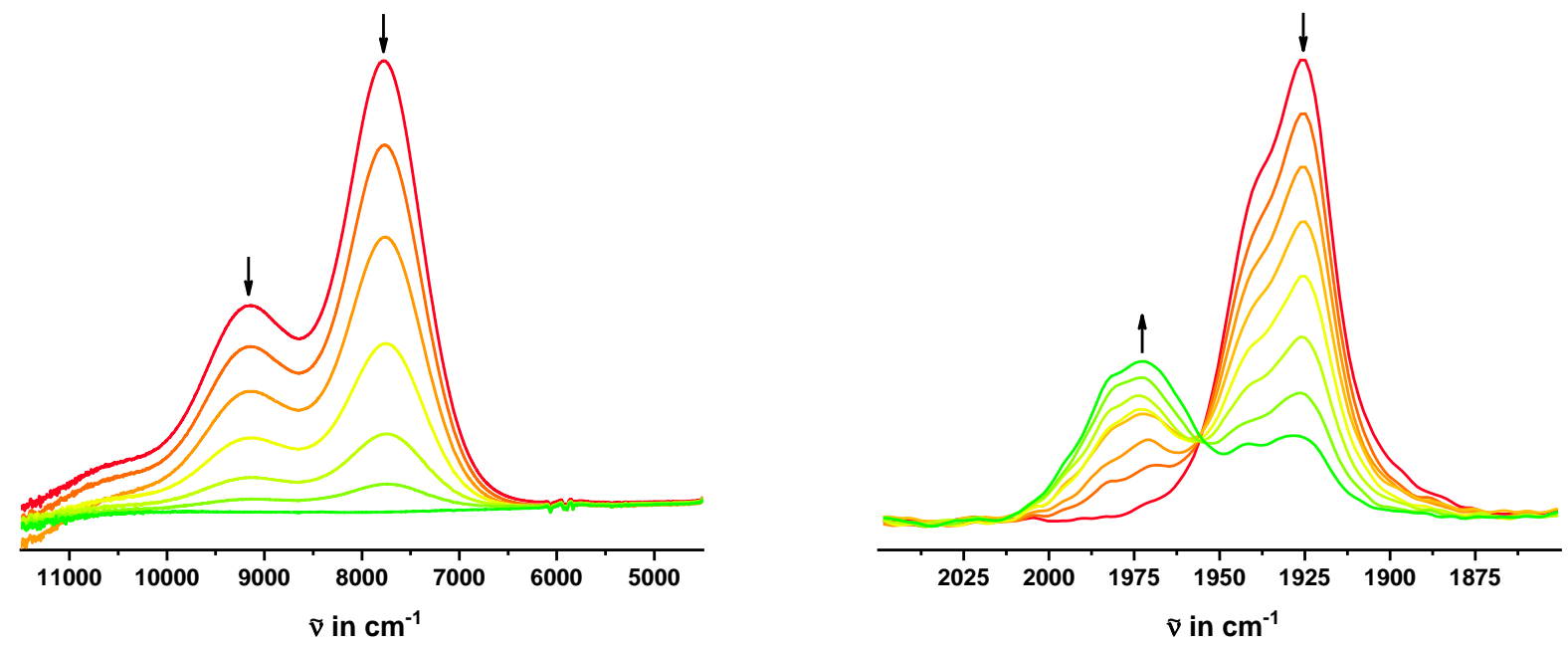

Figure S66. Changes in the IR spectra of $\mathbf{2}-\mathbf{B T E}^{2+}$ upon oxidation to $\mathbf{2}-\mathbf{B T E}^{4+}$, measured in $\mathrm{CH}_{2} \mathrm{Cl}_{2} /{ }^{n} \mathrm{Bu}_{4} \mathrm{NPF}_{6}$.
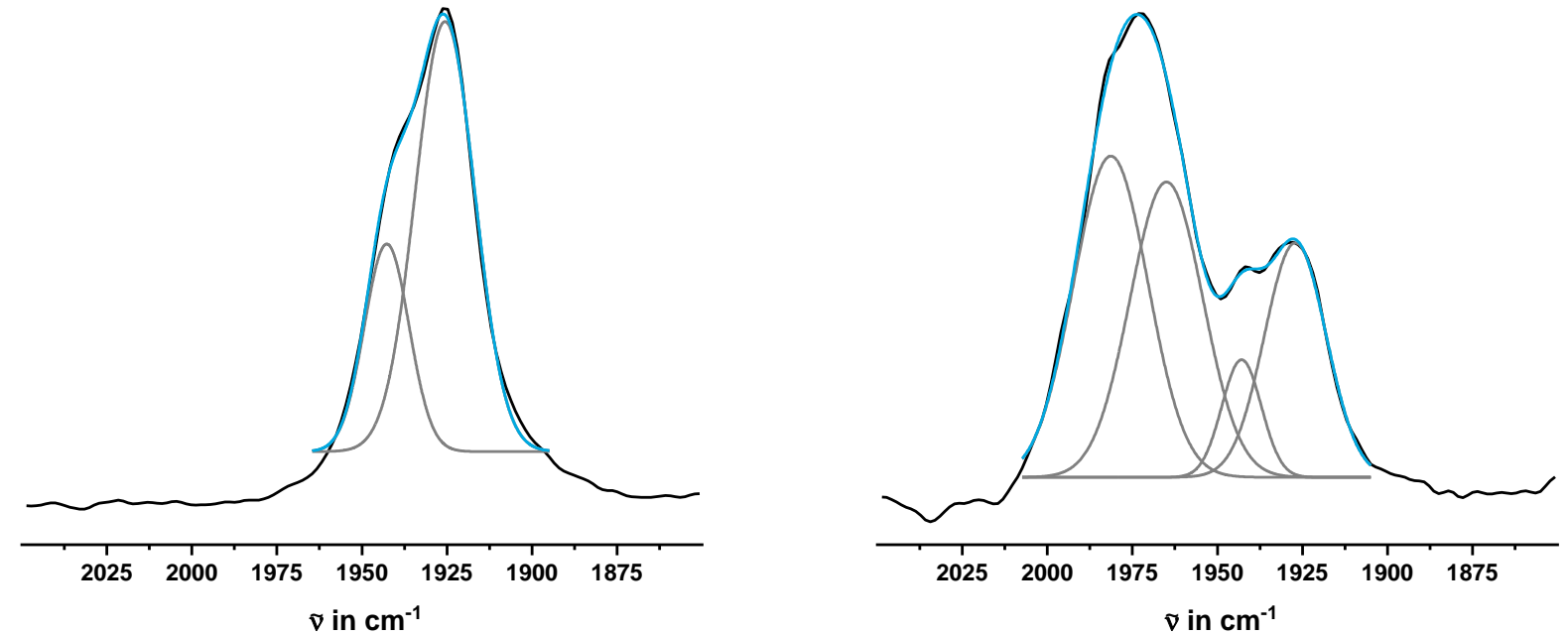

Figure S67. Deconvolutions of the Carbonyl bands of 2-BTE ${ }^{2+}$ (left) and 2-BTE ${ }^{4+}$ (right). 

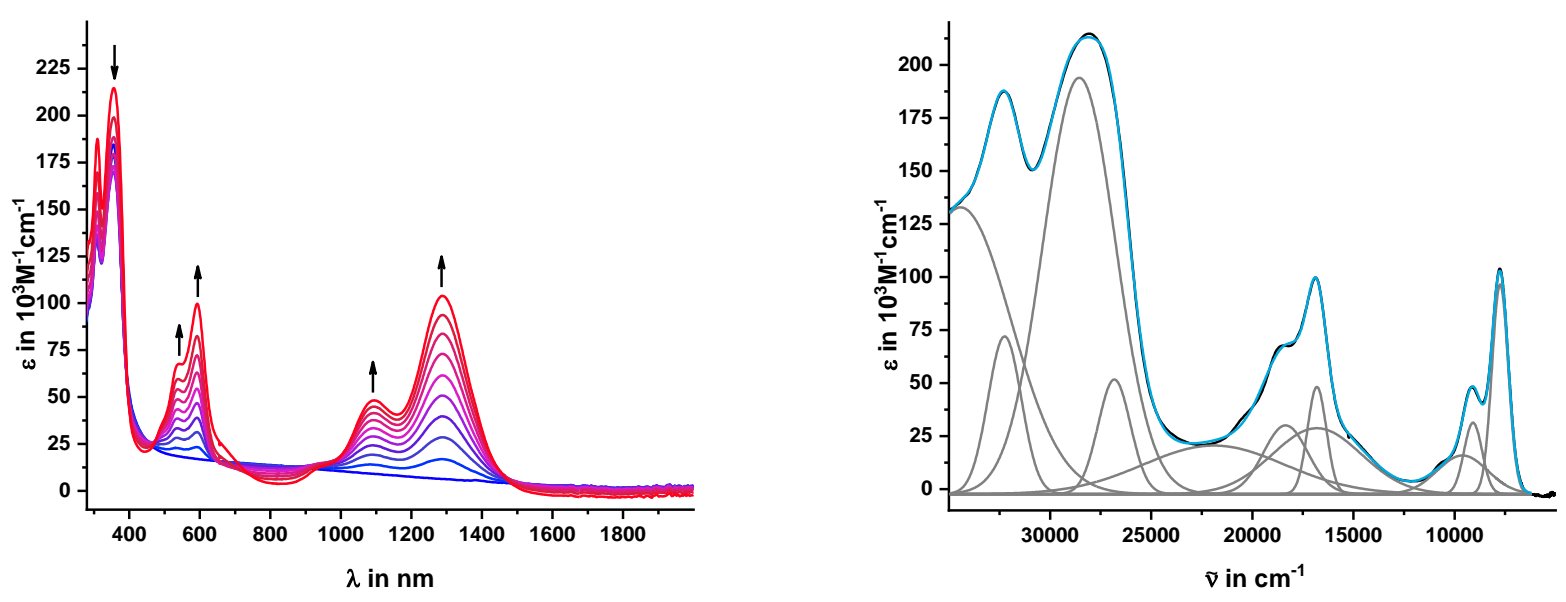

Figure S68. Changes in the UV/Vis/NIR spectra of 2-BTE ${ }^{2+}$ upon oxidation to 2-BTE ${ }^{4+}$ (left) and deconvolution (right), measured in $\mathrm{CH}_{2} \mathrm{Cl}_{2} /{ }^{n} \mathrm{Bu}_{4} \mathrm{NPF}_{6}$.
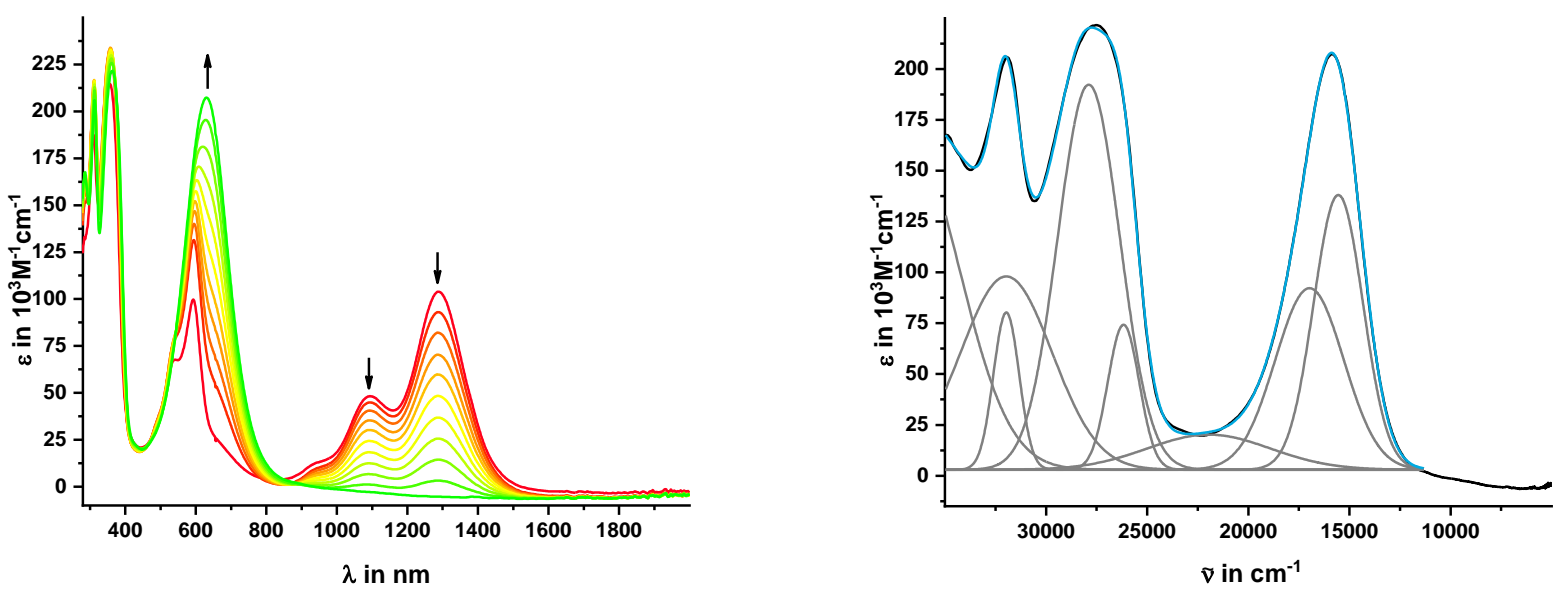

Figure S69. Changes in the UV/Vis/NIR spectra of 2-BTE ${ }^{2+}$ upon oxidation to 2-BTE ${ }^{4+}$ (left) and deconvolution (right), measured in $\mathrm{CH}_{2} \mathrm{Cl}_{2} /{ }^{n} \mathrm{Bu}_{4} \mathrm{NPF}_{6}$. 


\section{Compound 2-BN:}
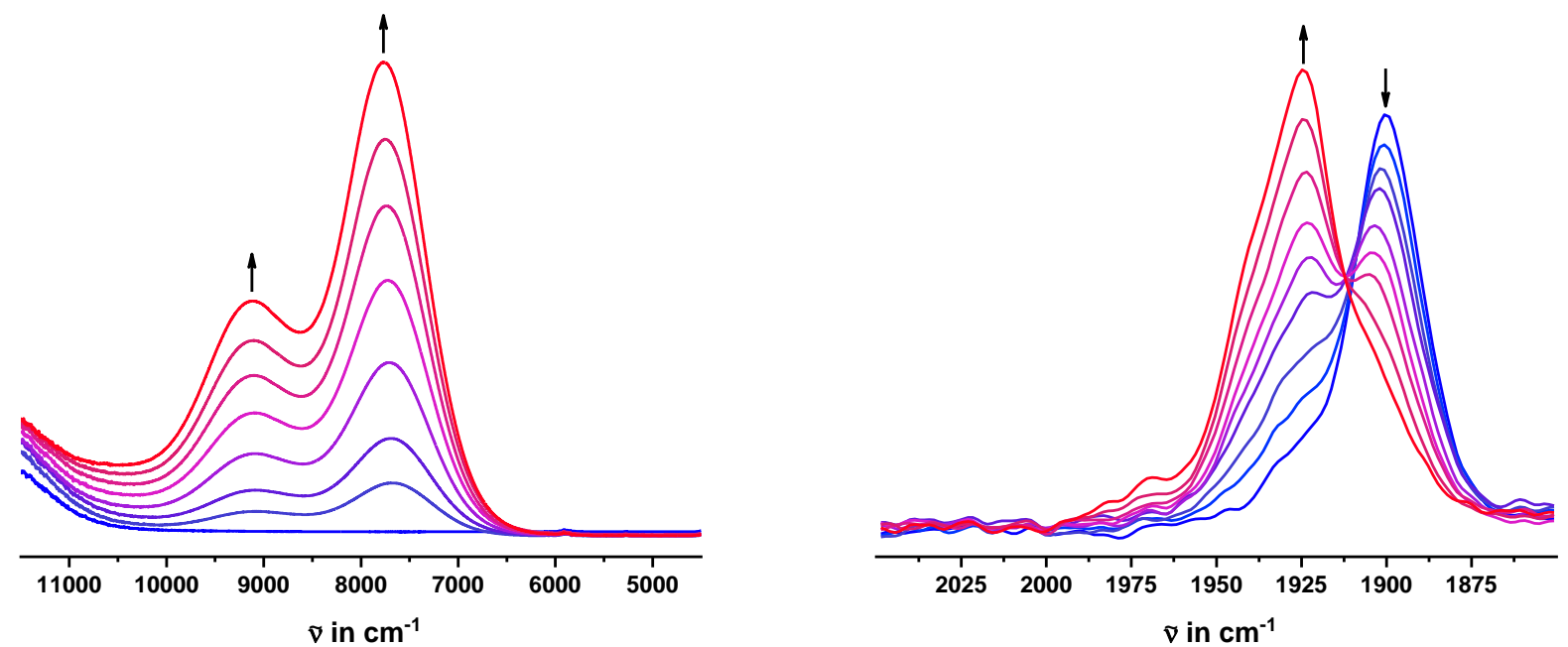

Figure S70. Changes in the IR spectra of $\mathbf{2 - B N}$ upon oxidation to $\mathbf{2}-\mathbf{B N}^{2+}$, measured in $\mathrm{CH}_{2} \mathrm{Cl}_{2} /{ }^{n} \mathrm{Bu}_{4} \mathrm{NPF}_{6}$.
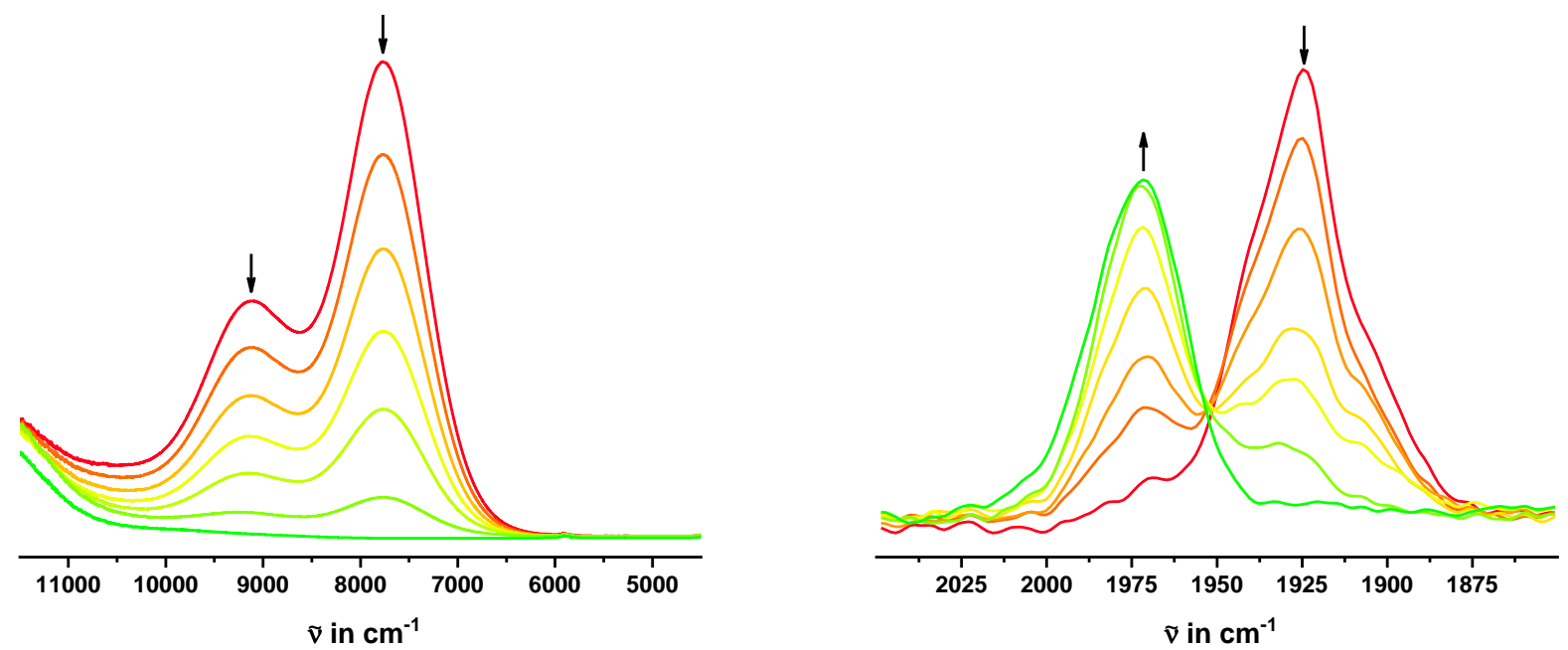

Figure S71. Changes in the IR spectra of $\mathbf{2}-\mathbf{B N}^{2+}$ upon oxidation to $\mathbf{2}-\mathbf{B} \mathbf{N}^{4+}$, measured in $\mathrm{CH}_{2} \mathrm{Cl}_{2} /{ }^{n} \mathrm{Bu}_{4} \mathrm{NPF}_{6}$.
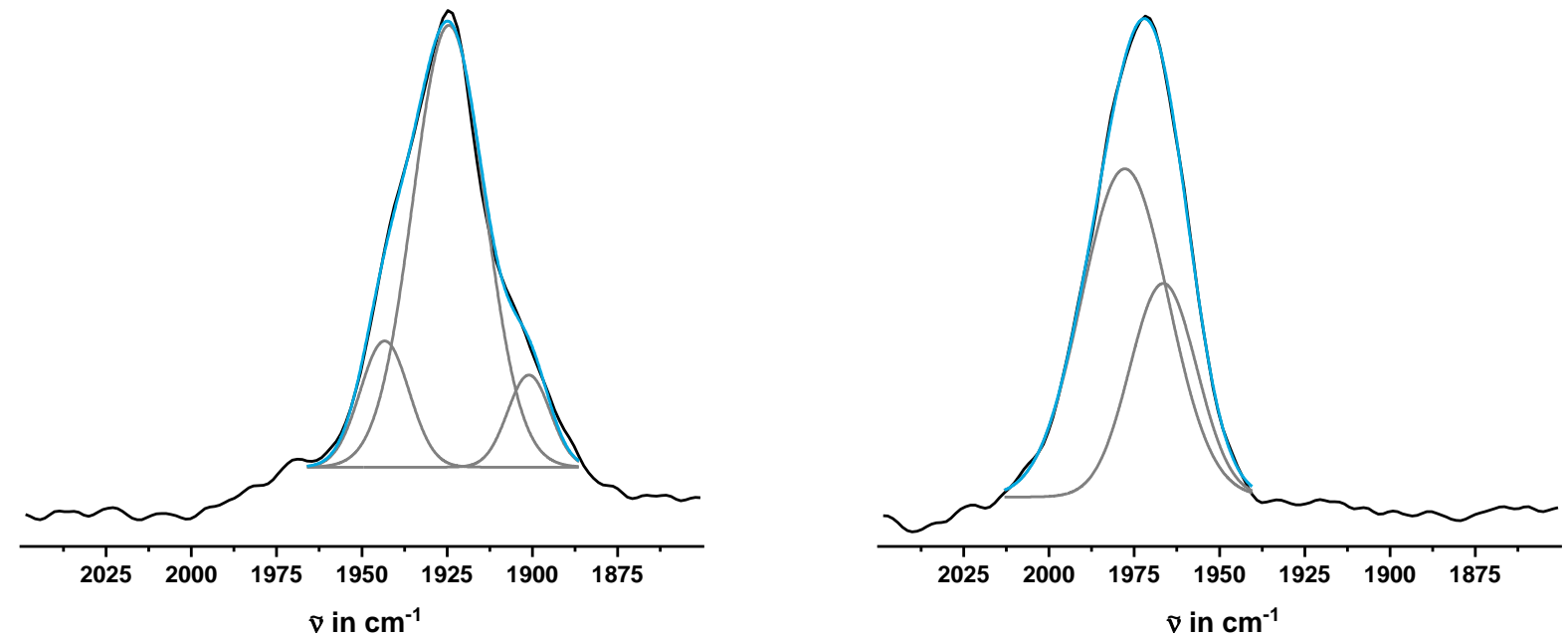

Figure S72. Deconvolutions of the Carbonyl bands of $\mathbf{2}-\mathbf{B N}^{2+}$ (left) and $\mathbf{2}-\mathbf{B N}^{4+}$ (right). 

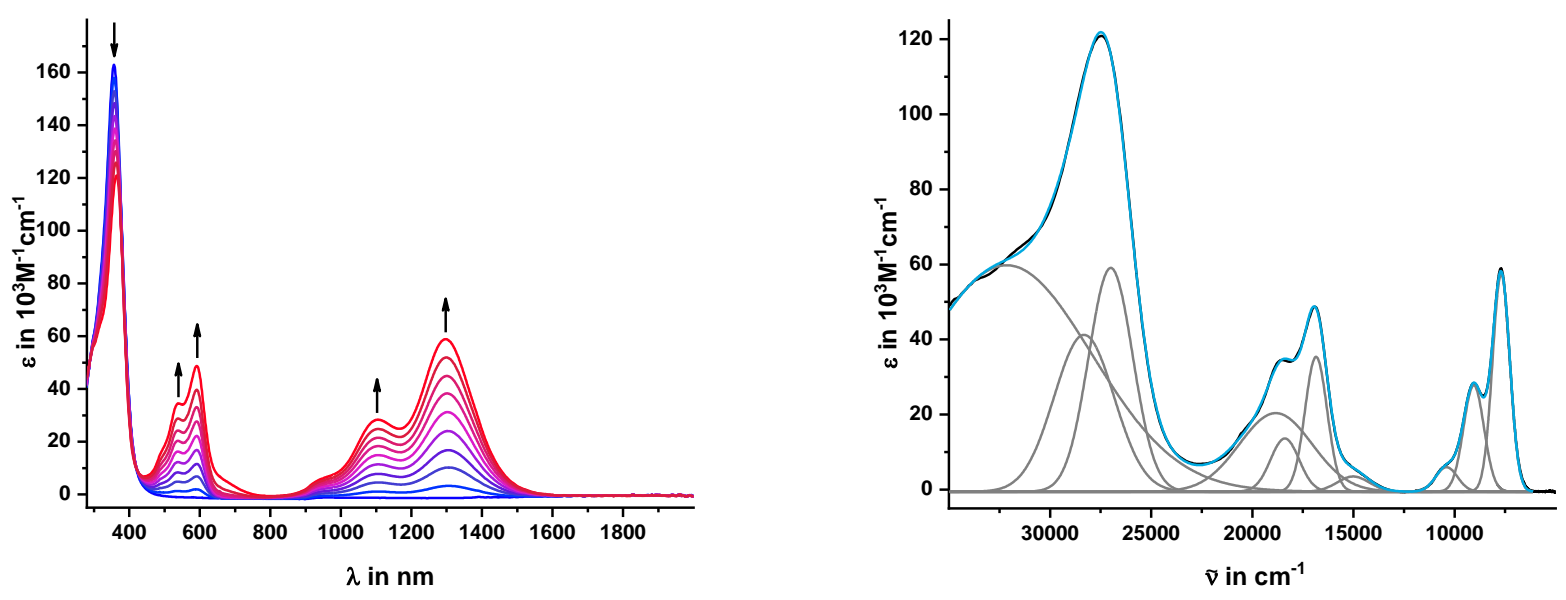

Figure S73. Changes in the UV/Vis/NIR spectra of $\mathbf{2}-\mathbf{B N}^{2+}$ upon oxidation to $\mathbf{2}-\mathbf{B N}^{4+}$ (left) and deconvolution (right), measured in $\mathrm{CH}_{2} \mathrm{Cl}_{2} /{ }^{n} \mathrm{Bu}_{4} \mathrm{NPF}_{6}$.
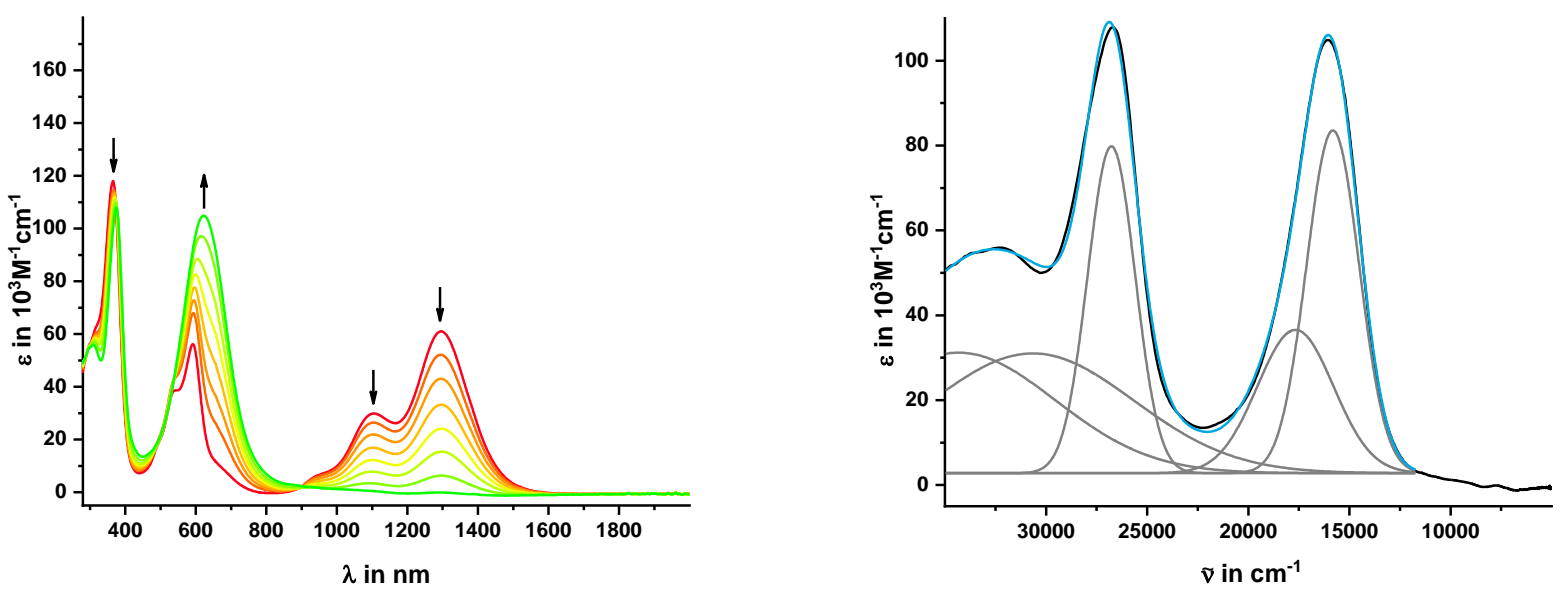

Figure S74. Changes in the UV/Vis/NIR spectra of $\mathbf{2}-\mathbf{B N}^{2+}$ upon oxidation to $\mathbf{2}-\mathbf{B N}^{4+}$ (left) and deconvolution (right), measured in $\mathrm{CH}_{2} \mathrm{Cl}_{2} /{ }^{n} \mathrm{Bu}_{4} \mathrm{NPF}_{6}$. 


\section{Compound 2-NB:}
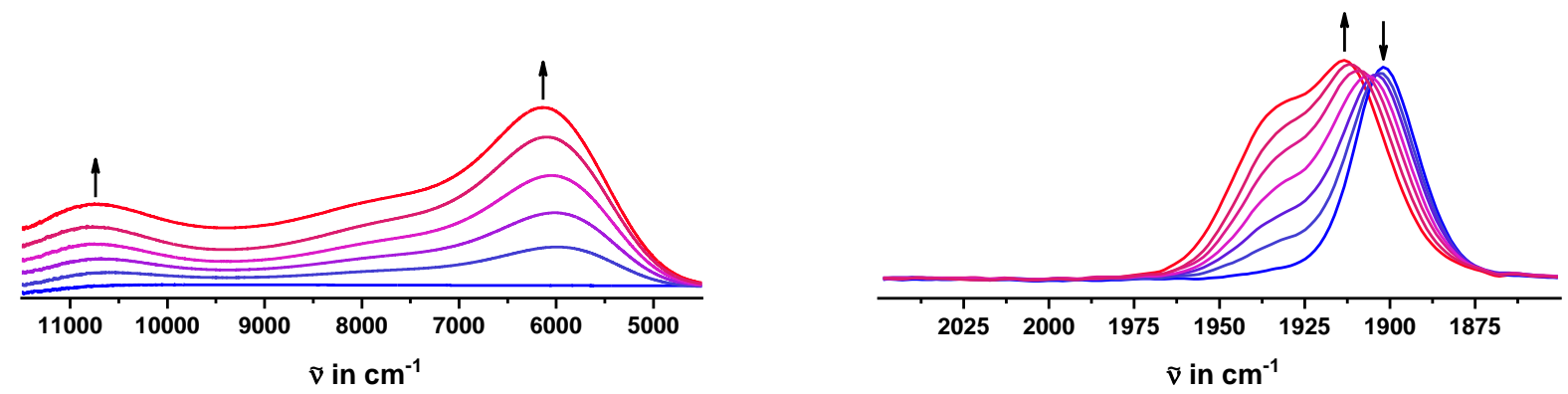

Figure S75. Changes in the IR spectra of $\mathbf{2 - N B}$ upon oxidation to $2-\mathbf{N B}^{2+}$, measured in $\mathrm{CH}_{2} \mathrm{Cl}_{2} /{ }^{n} \mathrm{Bu}_{4} \mathrm{NPF}_{6}$.
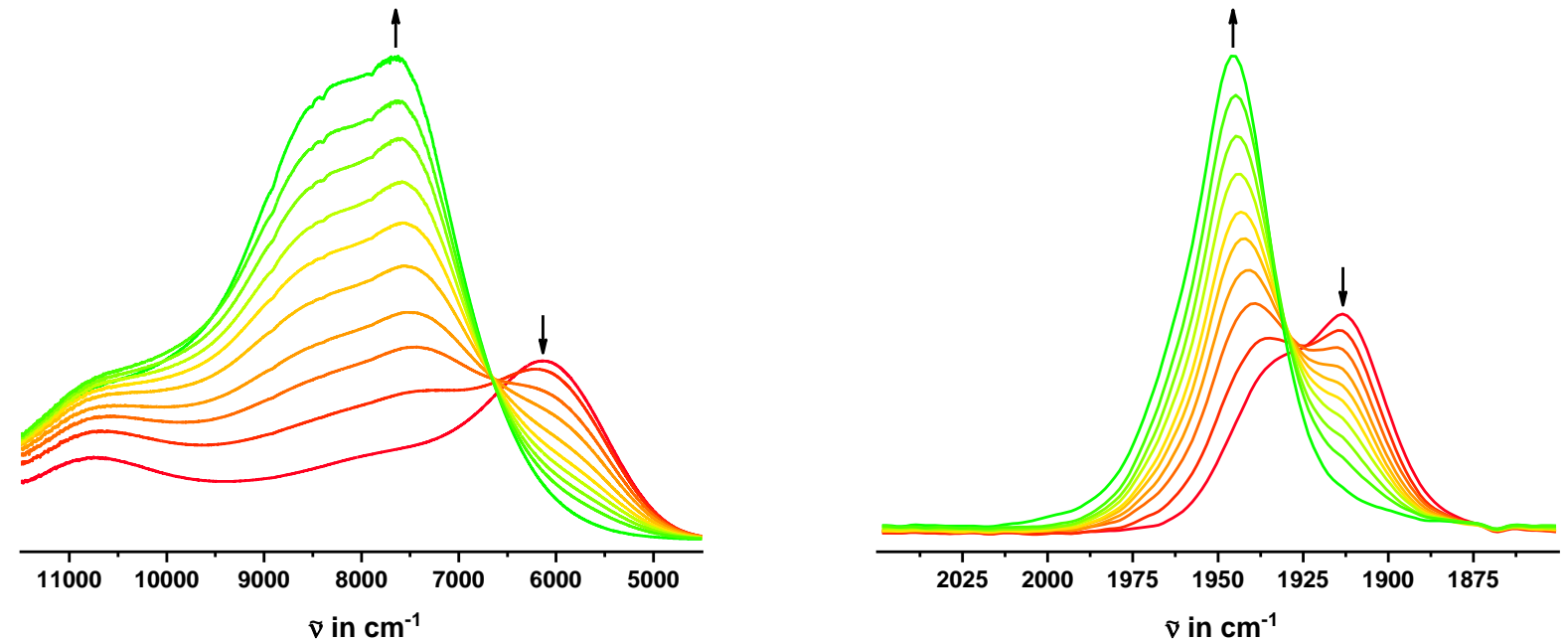

Figure S76. Changes in the IR spectra of $\mathbf{2}-\mathbf{N B}^{2+}$ upon oxidation to $\mathbf{2 - \mathbf { N B } ^ { 4 + }}$, measured in $\mathrm{CH}_{2} \mathrm{Cl}_{2} /{ }^{n} \mathrm{Bu}_{4} \mathrm{NPF}_{6}$.
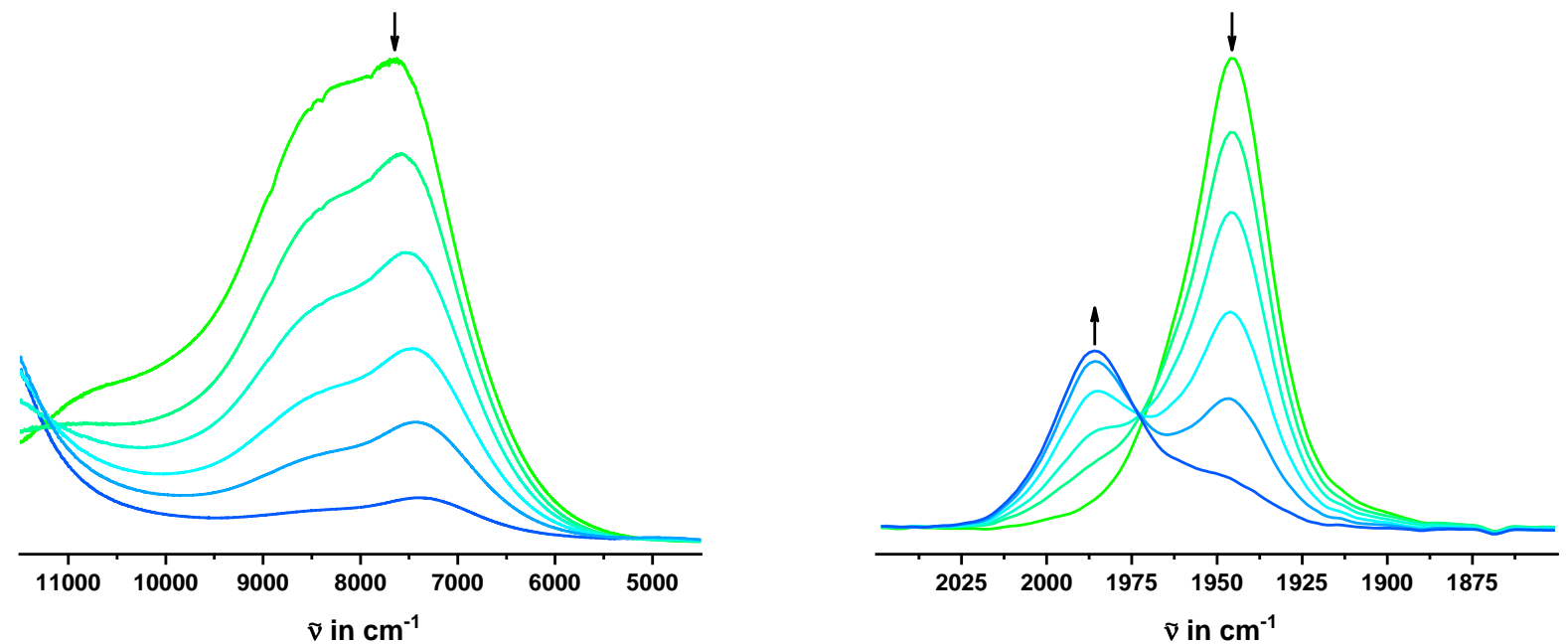

Figure S77. Changes in the IR spectra of $\mathbf{2 - \mathbf { N B } ^ { 4 + }}$ upon oxidation to $\mathbf{2 - \mathbf { N B } ^ { 6 + }}$, measured in $\mathrm{CH}_{2} \mathrm{Cl}_{2} /{ }^{n} \mathrm{Bu}_{4} \mathrm{NPF}_{6}$. 

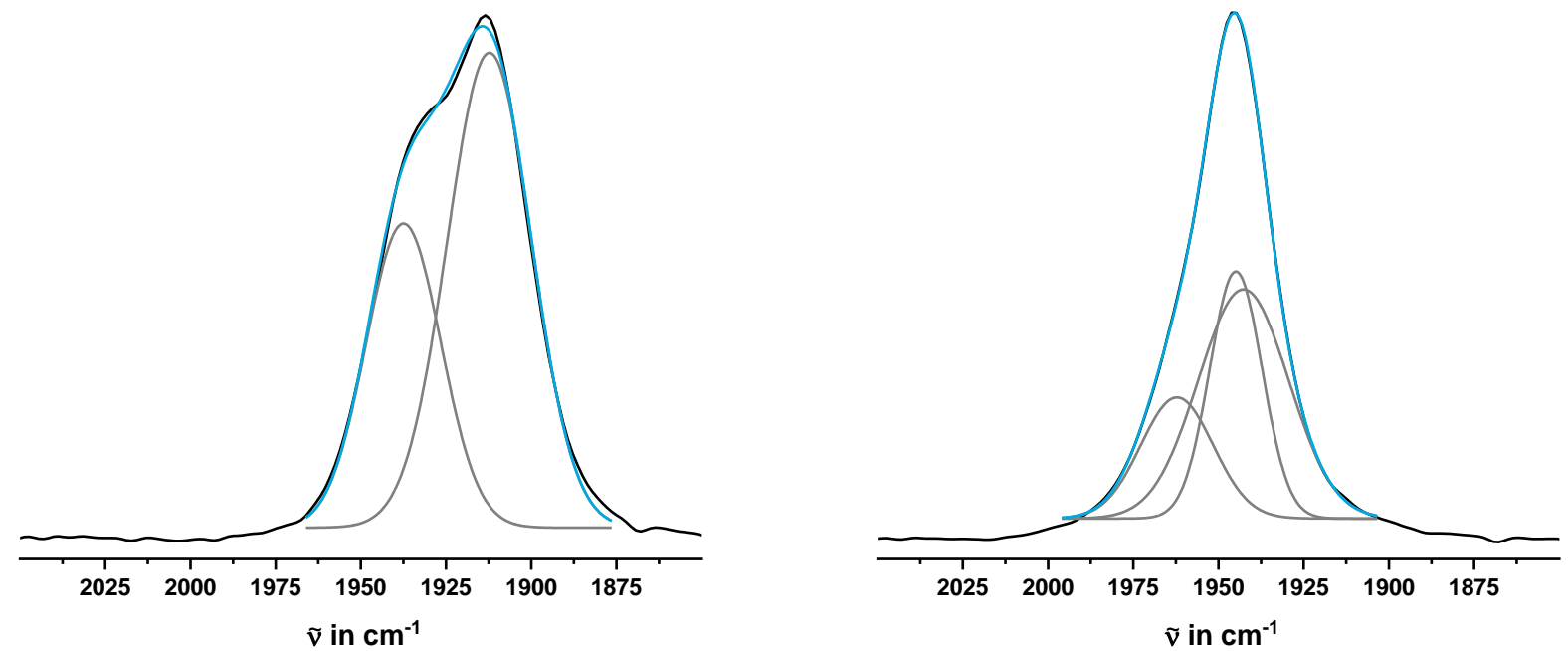

Figure S78. Deconvolutions of the Carbonyl bands of 2-NB ${ }^{2+}$ (left) and 2-NB ${ }^{4+}$ (right).
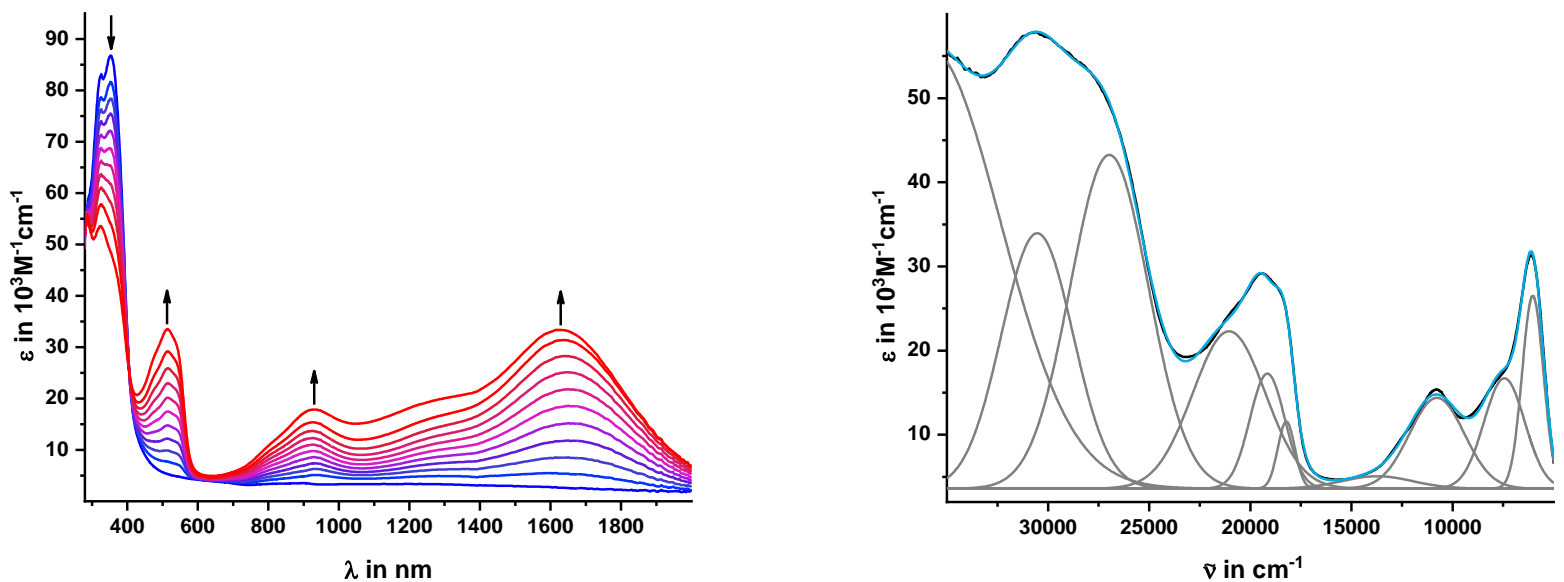

Figure S79. Changes in the UV/Vis/NIR spectra of 2-NB upon oxidation to 2-NB ${ }^{2+}$ (left) and deconvolution (right), measured in $\mathrm{CH}_{2} \mathrm{Cl}_{2} /{ }^{n} \mathrm{Bu}_{4} \mathrm{NPF}_{6}$.
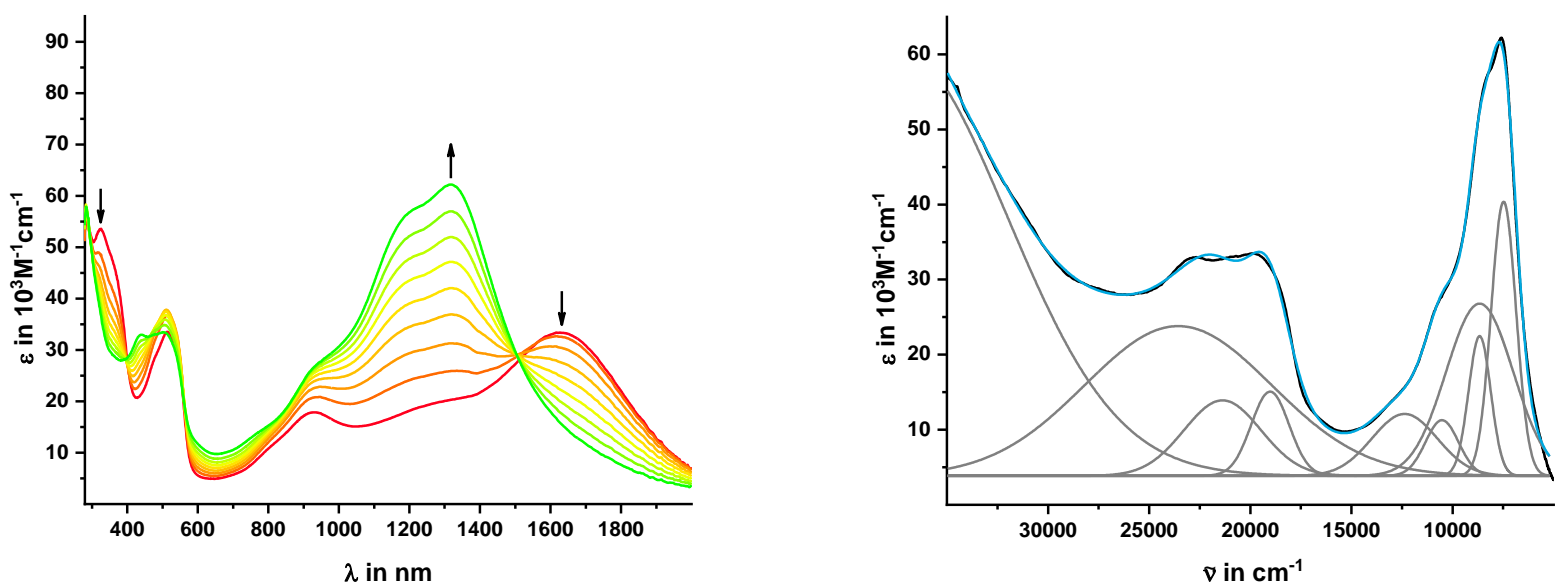

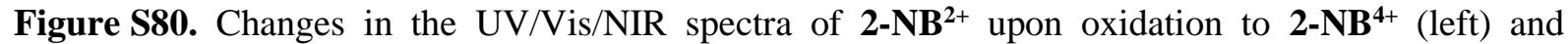
deconvolution (right), measured in $\mathrm{CH}_{2} \mathrm{Cl}_{2} /{ }^{n} \mathrm{Bu}_{4} \mathrm{NPF}_{6}$. 

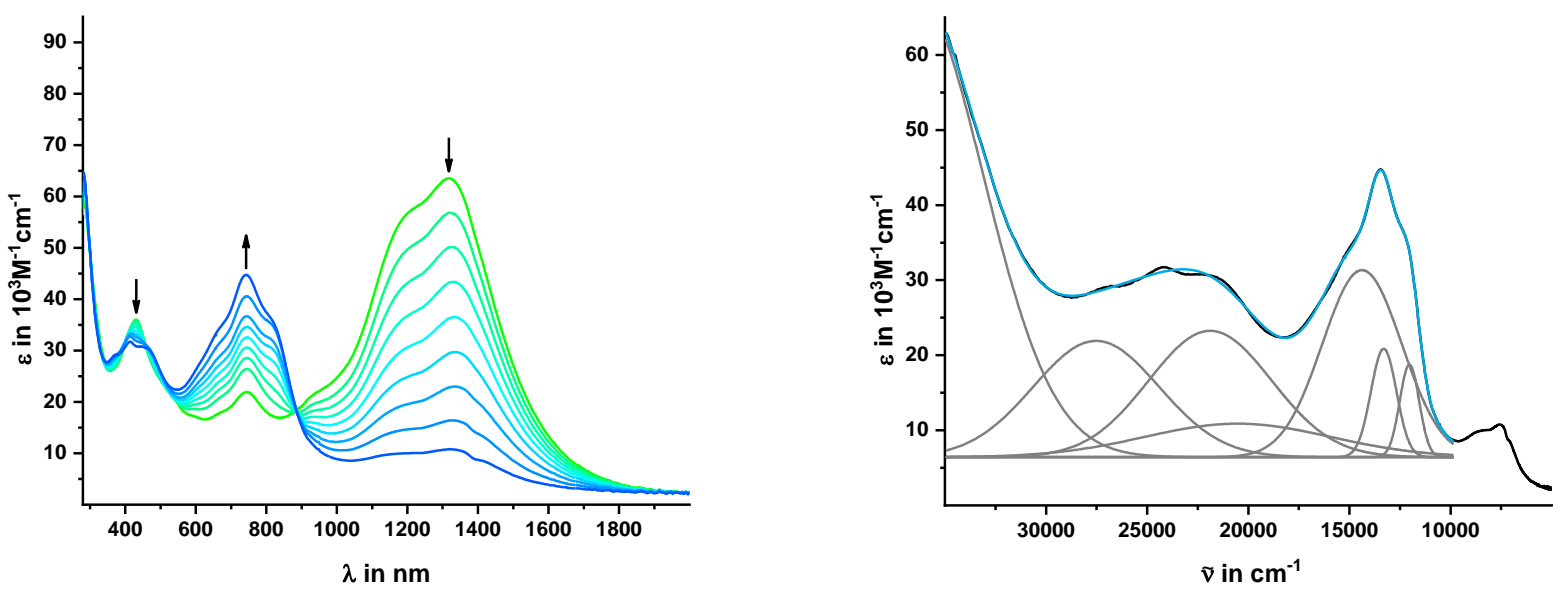

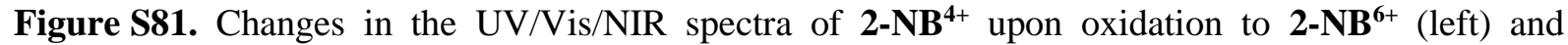
deconvolution (right), measured in $\mathrm{CH}_{2} \mathrm{Cl}_{2} /{ }^{n} \mathrm{Bu}_{4} \mathrm{NPF}_{6}$. 


\section{EPR Spectroscopy}

\section{Compound 2-BT:}
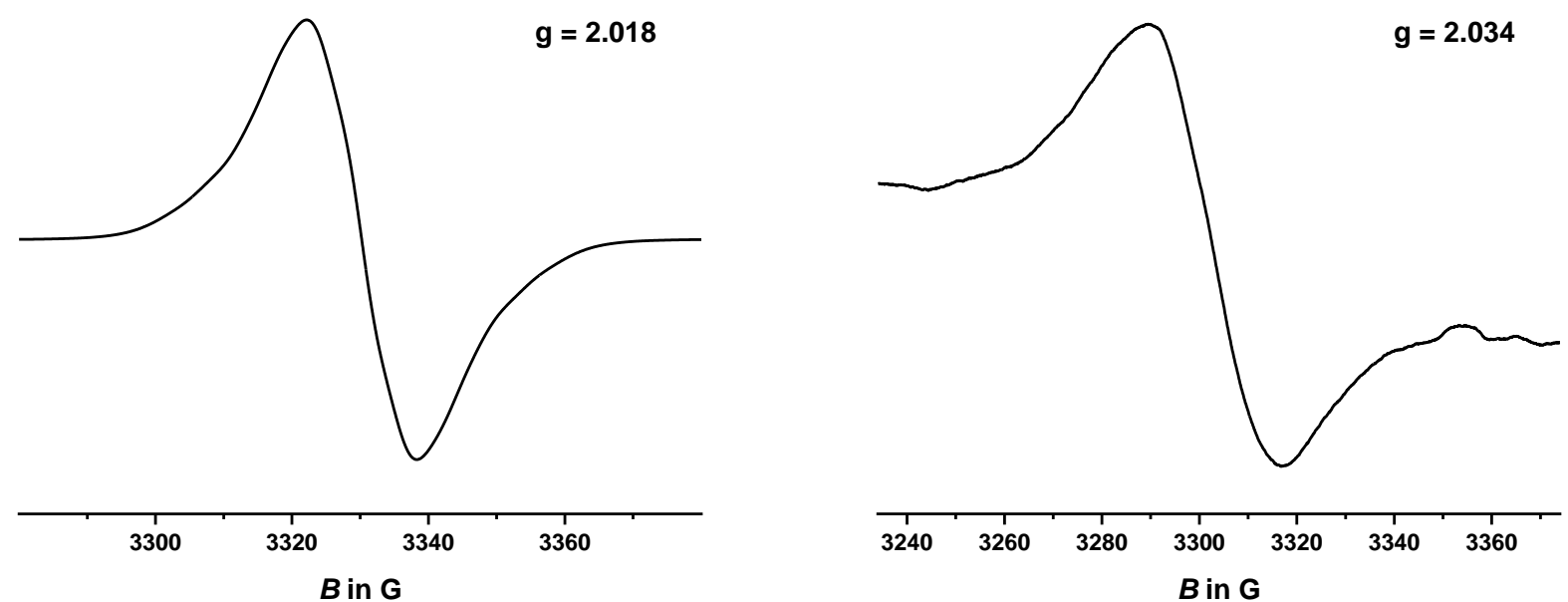

Figure S82. EPR spectra of 2-BT ${ }^{2+}$ (left) and $\mathbf{2}-\mathbf{B T}^{4+}$ (right) at room temperature.

\section{Compound 2-BTO:}
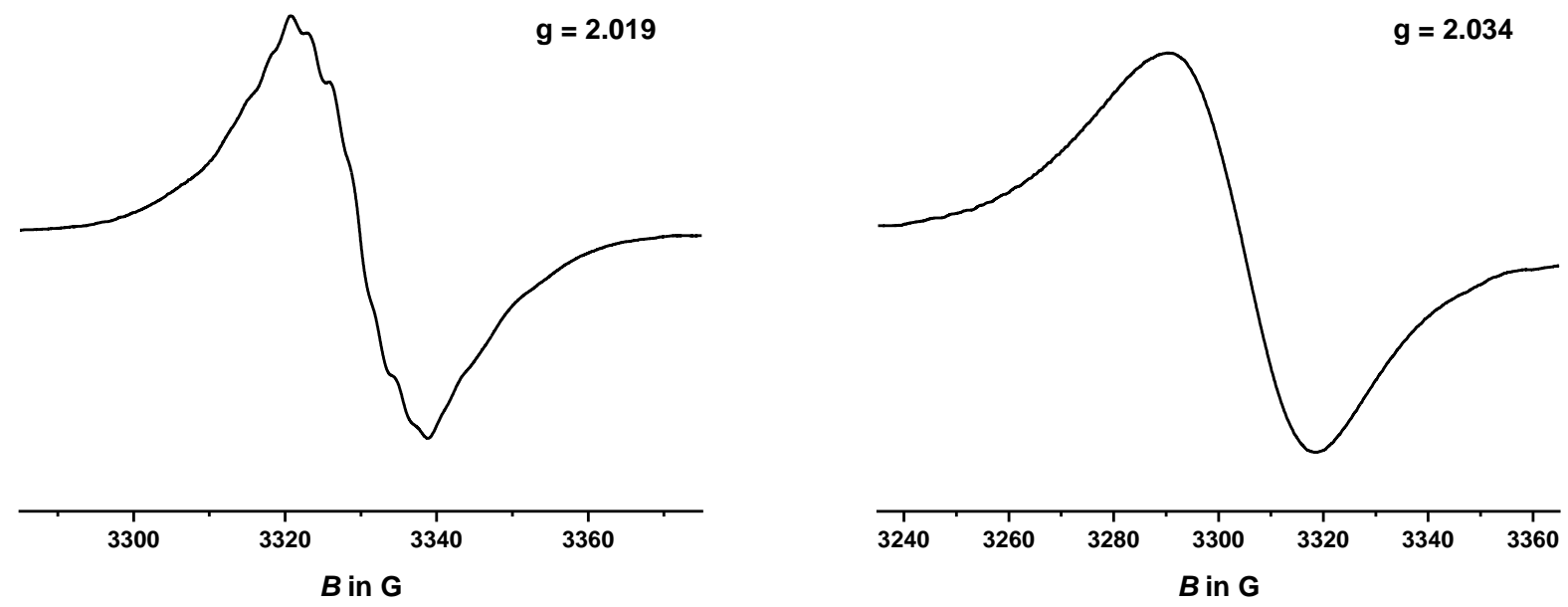

Figure S83. EPR spectra of 2-BTO ${ }^{2+}$ (left) and 2-BTO ${ }^{4+}$ (right) at room temperature.

\section{Compound 2-BTE:}
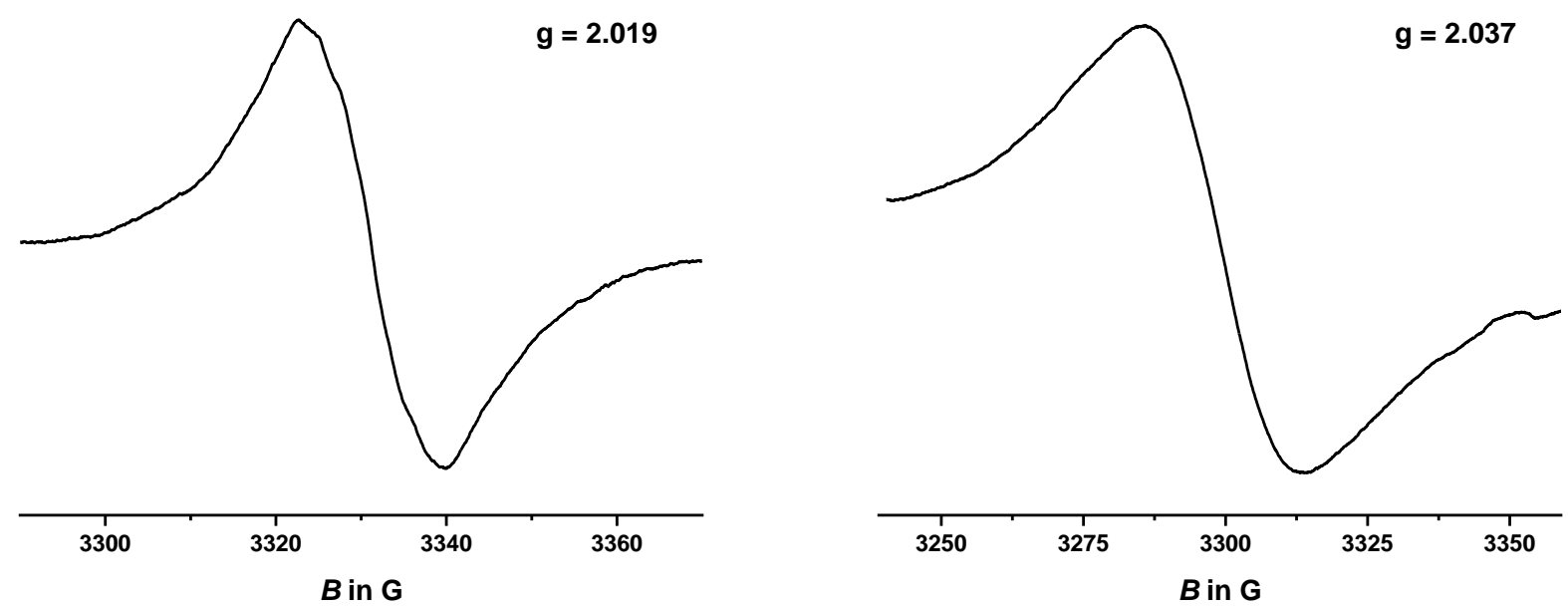

Figure S84. EPR spectra of 2-BTE ${ }^{2+}$ (left) and 2-BTE ${ }^{4+}$ (right) at room temperature. 


\section{Compound 2-BN:}
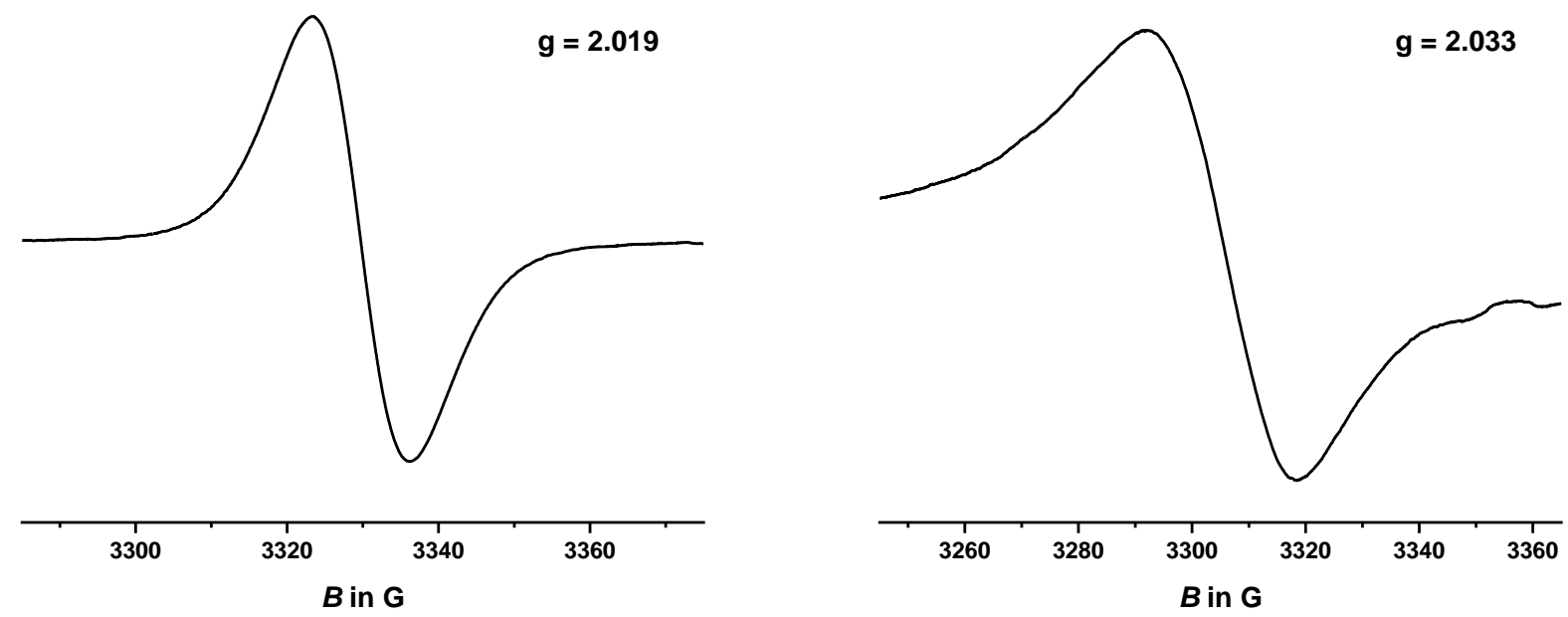

Figure S85. EPR spectra of 2-BN ${ }^{2+}$ (left) and 2-BN ${ }^{2+}$ (right) at room temperature.

\section{Compound 2-NB}
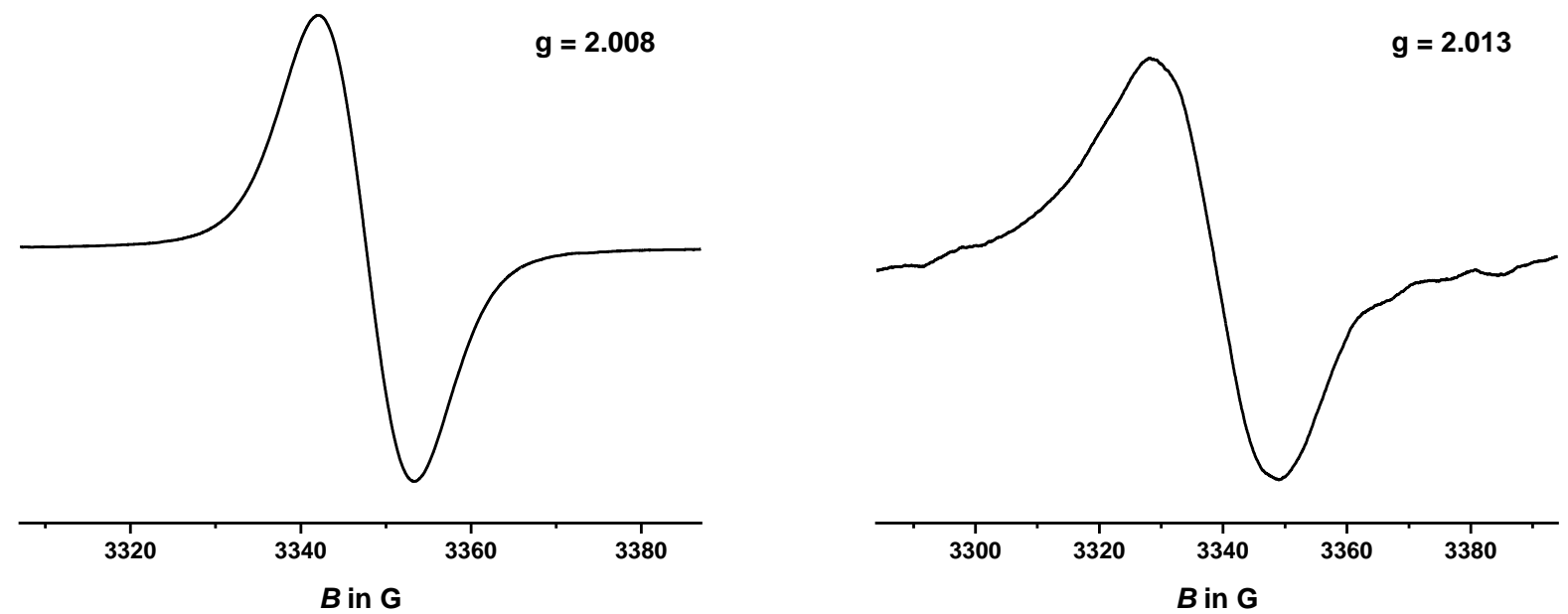

Figure S86. EPR spectra of $\mathbf{2}-\mathbf{N B}^{2+}$ (left) and $\mathbf{2}-\mathbf{N B}^{\mathbf{4 +}}$ (right) at room temperature.
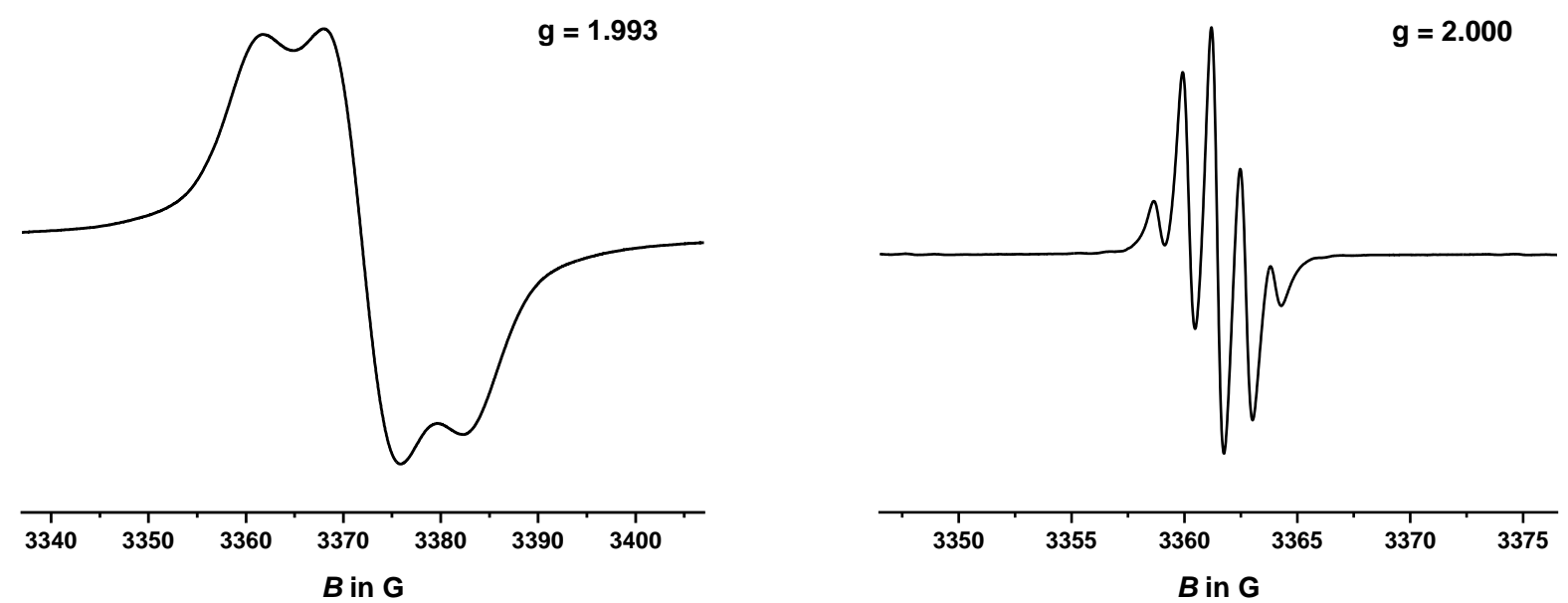

Figure S87. EPR spectra of 2-NB ${ }^{6+}$ (left) and of thianthrenium hexafluoroantimonate (right) at room temperature. 

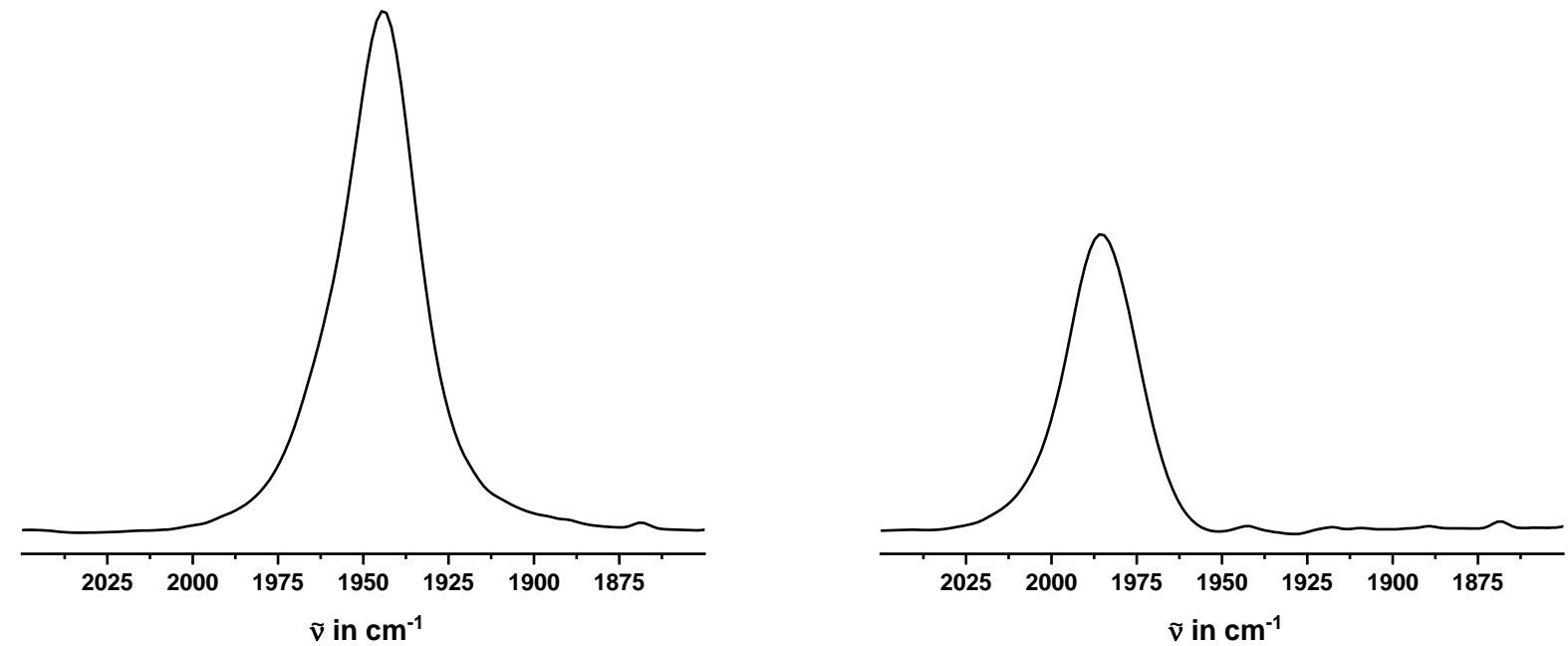

Figure S88. Carbonyl bands of 2-NB ${ }^{4+}$ (left) and $\mathbf{2}-\mathbf{N B}^{\mathbf{6}}$ (right) generated via chemical oxidation with the appropriate number of equivalents of thianthrenium hexafluoroantimonate in $\mathrm{CH}_{2} \mathrm{Cl}_{2}$.

\section{References}

(1) Dolomanov, O.V.; Bourhis, L. J.; Gildea, R. J.; Howard, J. A. K.; Puschmann, H. J. Appl. Cryst., 2009, 42 (2), 339-341.

(2) Sheldrick, G.M. Acta Cryst. 2015, A71, 3-8.

(3) Sheldrick, G.M. Acta Cryst. 2015, C71, 3-8. 